\title{
The Multiplicity of M Dwarfs in Young Moving Groups
}

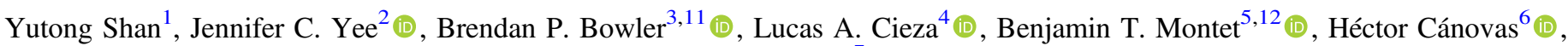 \\ Michael C. $\operatorname{Liu}^{7}$ (D), \\ and \\ Laird M. Close ${ }^{8}$ (D) Phil M. Hinz ${ }^{8}$, Jared R. Males ${ }^{8,12}$ (D), Katie M. Morzinski ${ }^{8,12}$ (D), Amali Vaz ${ }^{8}$, \\ Vanessa P. Bailey ${ }^{9}$, and Katherine B. Follette ${ }^{10}$ (iD \\ (MagAO Team) \\ ${ }^{1}$ Department of Astronomy, Harvard University, 60 Garden Street, Cambridge, MA 02138, USA \\ ${ }^{2}$ Smithsonian Astrophysical Observatory, 60 Garden Street, Cambridge, MA 02138, USA \\ ${ }^{3}$ McDonald Observatory and the Department of Astronomy, The University of Texas at Austin, Austin, TX 78712, USA \\ ${ }^{4}$ Universidad Diego Portales, Facultad de Ingeniería y Ciencias, Avenida Ejército 441, Santiago, Chile \\ ${ }^{5}$ Department of Astronomy and Astrophysics, University of Chicago, 5640 S. Ellis Avenue, Chicago, IL 60637, USA \\ ${ }^{6}$ Departamento de Física Teórica, Universidad Autónoma de Madrid, Cantoblanco, E-28049 Madrid, Spain \\ ${ }^{7}$ Institute for Astronomy, University of Hawaii, 2680 Woodlawn Drive, Honolulu, HI 96822, USA \\ ${ }^{8}$ Steward Observatory, University of Arizona, 933 North Cherry Avenue, Tucson, AZ 85721, USA \\ ${ }^{9}$ Kavli Institute for Particle Astrophysics and Cosmology, Stanford University, Stanford, CA 94305, USA \\ ${ }^{10}$ Physics and Astronomy Department, Amherst College, 21 Merrill Science Drive, Amherst, MA 01002, USA \\ Received 2017 June 20; revised 2017 August 2; accepted 2017 August 2; published 2017 September 5
}

\begin{abstract}
We image 104 newly identified low-mass (mostly M-dwarf) pre-main sequence (PMS) members of nearby young moving groups (YMGs) with Magellan Adaptive Optics (MagAO) and identify 27 stellar binaries with instantaneous projected separation as small as 40 mas. Fifteen were previously unknown. The total number of multiple systems in this sample including spectroscopic and visual binaries from the literature is 36 , giving a raw stellar multiplicity rate of at least $35_{-4}^{+5} \%$ for this population. In the separation range of roughly $1-300$ au in which infrared AO imaging is most sensitive, the raw multiplicity rate is at least $24_{-4}^{+5} \%$ for binaries resolved by the MagAO infrared camera (Clio). The M-star subsample of 87 stars yields a raw multiplicity of at least $30_{-4}^{+5} \%$ over all separations, $21_{-4}^{+5} \%$ for secondary companions resolved by Clio from 1 to 300 au $\left(23_{-4}^{+5} \%\right.$ for all known binaries in this separation range). A combined analysis with binaries discovered by the Search for Associations Containing Young stars shows that stellar multiplicity fraction as a function of mass over the range of 0.2 to $1.2 M_{\odot}$ appears to be linearly flat, in contrast to the field, where multiplicity increases with mass. After bias corrections are applied, the multiplicity of low-mass YMG members $\left(0.2-0.6 M_{\odot}\right)$ is in excess of the field. The overall multiplicity fraction is also consistent with being constant in age and across YMGs, which suggests that multiplicity rates for this mass range are largely set by $10 \mathrm{Myr}$ without appreciable evolution thereafter.
\end{abstract}

Key words: binaries: visual - methods: observational - methods: statistical - stars: low-mass - stars: pre-main sequence - techniques: photometric

Supporting material: machine-readable tables

\section{Introduction}

Young moving groups (YMGs) are groups comprising several dozens of coeval, kinematically associated stars that share a common birth environment. Typically aged 10-150 Myr, they are in the process of dispersing into the field (Zuckerman \& Song 2004; Torres et al. 2008). This population represents an excellent laboratory for directly imaging giant planets (e.g., Delorme et al. 2013; Bowler et al. 2017).

Because stars from a given YMG formed from the same collapsing gas cloud, they offer an opportunity to directly test stellar evolution models (e.g., Zuckerman \& Song 2004; Yee \& Jensen 2010; Jeffries et al. 2017). In particular, the binary systems among them are important for these tests in three ways. First, it is often possible to recover more detailed information on individual binaries, such as dynamical mass measurements, making them a stronger test of stellar evolution (e.g., Azulay

\footnotetext{
${ }^{11}$ NASA Hubble Fellow.

12 NASA Sagan Fellow.
}

et al. 2015; Montet et al. 2015). Second, when considering an ensemble of YMG members, it is important to have accurate properties, which might be inferred improperly if a close binary is thought to be a single star. Finally, binarity information is crucial to reaching dependable YMG membership conclusions, since unresolved binaries may affect the measured photometry as well as the radial velocity of a system used for its YMG assignment (e.g., Malo et al. 2013; Kraus et al. 2014).

In addition, the multiplicity of YMGs themselves may provide important insight into the formation and evolution of binary star systems. In general, primordial binarity could play an important role in protostellar disk evolution as well as planet formation (Cieza et al. 2009; Kraus et al. 2012, 2016). Furthermore, YMG members are representative of a transitional stage between stars in their birth clusters and those in the field (López-Santiago et al. 2006).

The Search for Associations Containing Young stars (SACY) consortium has been actively studying the properties of stars in nearby YMGs (Torres et al. 2008). Based on their 


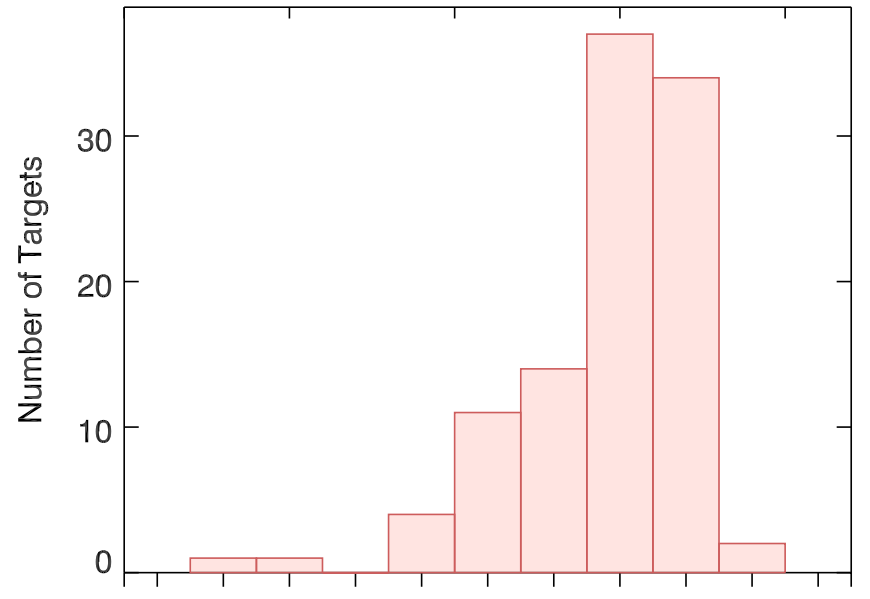

$\begin{array}{llllllllll}\text { G6 } & \mathrm{K} 0 & \mathrm{~K} 2 & \mathrm{~K} 4 & \mathrm{~K} 6 & \mathrm{M} 0 & \mathrm{M} 2 & \mathrm{M} 4 & \mathrm{M} 6\end{array}$

Figure 1. Distribution of spectral types for our observed targets. They are chiefly pre-main sequence $\mathrm{K}$ and $\mathrm{M}$ dwarfs.

survey of FGK-dwarfs, Elliott et al. (2014, 2015, 2016) find that stellar multiplicity is independent of primary mass. This result is consistent with findings from studies of very young star-forming regions (Leinert et al. 1993; Köhler et al. 2000), as well as young (5 Myr) OB associations (Lafrenière et al. 2014), but very different from what is observed in the field (e.g., Duchêne \& Kraus 2013, and references therein).

In this work, we present adaptive optics (AO) imaging for recently discovered nearby $\mathrm{K}$ - and M-type YMG members that complement the multiplicity study of Elliott et al. (2014, 2015, 2016). We identify visual binaries from 1-300 au and examine their overall multiplicity fractions as a function of mass, age, and YMG, as well as distributions in separation and mass ratio.

This paper is organized as follows. The sample is presented in Section 2. We document our observations and the data reduction procedure in Section 3. Section 4 describes the binaries in our observed targets. The visual binary (VB) discoveries are validated through, among other methods, comoving analysis combined with measurements from the literature (Section 4.1.1). We perform multiplicity statistics measurements on our sample in Section 5. Therein we first describe the construction of detection completeness maps (Section 5.1) and then the evaluation of physical parameters for the systems (Section 5.2). From there we calculate overall raw multiplicity fractions (Section 5.3) and distributions in separation and mass ratio for our sample (Section 5.4). In Section 5.5 we combine our sample with that of SACY for a joint analysis. This extends YMG multiplicity fraction measurements from 0.2 to $1.2 M_{\odot}$. Possible biases and their effects are discussed at length in Section 5.6. We conclude with Section 6.

\section{A Sample of Low-mass YMG Members}

Our target list is drawn primarily from Malo et al. (2013, 2014) (64 targets) and Kraus et al. (2014) (34 targets), and it is supplemented by targets from Moór et al. (2013) (5 targets) and Rodriguez et al. (2013) (2 targets). Stars in these catalogs are members of young $(<200 \mathrm{Myr})$ local $(\lesssim 100 \mathrm{pc})$ YMGs. In selecting targets, we had a strong preference for late-type dwarfs (K5V-M5V, see Figure 1) with $\delta<-40^{\circ}$ and $H<11 \mathrm{mag}$. From these catalogs, we eliminated many of the known visual binaries with separately resolvable spectral types. Since the selection strategy is not statistically uniform, we discuss the effects of potential biases in Section 5.6.2. We do
Table 1

Properties of Young Moving Groups Contributing to Our Sample

\begin{tabular}{lcccr}
\hline \hline Group & $\begin{array}{c}\text { Age } \\
(\mathrm{Myr})\end{array}$ & $\begin{array}{c}\text { Isochrone } \\
\text { Age }^{\mathrm{a}} \\
(\mathrm{Myr})\end{array}$ & $\begin{array}{c}\text { Dist. } \\
\text { Range }^{\mathrm{b}} \\
(\mathrm{pc})\end{array}$ & $\begin{array}{r}\text { Number } \\
\text { of Stars }^{\text {Imaged }}\end{array}$ \\
\hline $\begin{array}{l}\text { Tucana-Horologium } \\
\text { (TucHor) }\end{array}$ & $41-49$ & 40 & $36-71$ & $51(2)$ \\
Columba (Col) & $38-48$ & 40 & $35-81$ & $15(2)$ \\
$\beta$ Pictoris (bPic) & $21-27$ & 25 & $9-73$ & $13(1)$ \\
Argus (Arg) & $30-50$ & 40 & $8-68$ & $10(1)$ \\
AB Doradus & $130-200$ & 120 & $7-77$ & $6(2)$ \\
(ABDor) & $38-56$ & 50 & $46-88$ & $6(0)$ \\
Carina (Car) & $7-13$ & 10 & $28-92$ & $4(1)$ \\
TW Hydrae (TWA) & & & & \\
\hline
\end{tabular}

Notes.

${ }^{a}$ The ages of the closest isochrones from Baraffe et al. (2015) adopted for stellar mass calculations, see Section 4.1.

b YMG distance ranges are from Table 1 in Malo et al. (2013).

${ }^{\mathrm{c}}$ Unbracketed value denotes the total number of observed targets attributed to the group. In parentheses are the number of targets that have non-negligible probability (i.e., $>10 \%$ ) of belonging to another YMG.

Table 2

Summary of the MagAO Observing Runs

\begin{tabular}{llc}
\hline \hline Date & Filter (Clio) & \# of Images \\
\hline 2014 Apr 17-21 & H & 4 \\
2014 Nov 10-12 & Ks & 4 \\
2014 Nov 30-Dec 2 & H or Ks & 4 \\
2015 May 10-12 & H & 4 \\
2015 Nov 26-27 & H & 8 \\
\hline
\end{tabular}

not take into account whether a target is a known spectroscopic binary (SB). At the time of observation, most of these targets had not been previously imaged with AO.

Table 1 displays properties of the seven YMGs with members included in this study. The age for each YMG comes from Table 2 in Bell et al. (2015), except for Argus, whose age is from Table 1 in Malo et al. (2013).

Janson et al. (2017, hereafter J17) independently surveyed southern YMGs and published a catalog of low-mass visual binaries among them. The J17 observations were performed using lucky imaging in the $z^{\prime}$ band on the $3.5 \mathrm{~m}$ ESO New Technology telescope, with very comparable angular resolution sensitivity. There are 56 targets in common between J17 and our sample. Twenty-four of our targets were also previously observed with lucky imaging by Janson et al. (2012) (hereafter J12).

\section{Observations and Data}

\subsection{MagAO Observations}

Adaptive optics observations were conducted on the $6.5 \mathrm{~m}$ Magellan Clay Telescope at the Las Campanas Observatory in Chile using the MagAO instrument (Morzinski et al. 2014). Images were taken with two science cameras simultaneously: Clio in the near-infrared, and VisAO in the optical. Since late-type dwarfs are brighter in the near-infrared (NIR), we use Clio images for our science analysis. We allocate the visible light as follows: $96 \%$ is directed toward the wavefront sensor, whereas VisAO receives the remaining $4 \%$. Consequently, VisAO images tend to have a poor signal-to-noise ratio $(\mathrm{S} / \mathrm{N})$ and are only used to vet some close binaries. 
Table 3

YMG Low-mass Target Sample

\begin{tabular}{|c|c|c|c|c|c|c|c|c|c|c|}
\hline $\begin{array}{l}\text { Target } \\
\text { 2MASS ID }\end{array}$ & $\begin{array}{l}\text { Abbrv. } \\
\text { Name }\end{array}$ & R.A. & Decl. & $\mathrm{SpT}$ & $\begin{array}{l}\text { Distance }^{b} \\
(\mathrm{pc})\end{array}$ & References & $J$ & $H$ & $K$ & $\begin{array}{r}M_{\text {prim }}{ }^{\mathrm{g}} \\
\left(M_{\odot}\right)\end{array}$ \\
\hline \multicolumn{11}{|c|}{ Tucana-Horologium (TucHor) } \\
\hline J00125703-7952073 & J0012-7952 & $00: 12: 57.03$ & $-79: 52: 07.3$ & M2.9 & $48 \pm 2$ & 3 & 9.68 & 9.05 & 8.75 & 0.49 \\
\hline J00144767-6003477 & J0014-6003 & $00: 14: 47.67$ & $-60: 03: 47.7$ & M3.6 & $42 \pm 2$ & 3 & 9.71 & 9.10 & 8.83 & 0.39 \\
\hline J00152752-6414545 & J0015-6414 & $00: 15: 27.52$ & $-64: 14: 54.5$ & M1.8 & $50 \pm 3$ & 3 & 9.32 & 8.69 & 8.44 & 0.60 \\
\hline J00171443-7032021 & J0017-7032 & $00: 17: 14.43$ & $-70: 32: 02.1$ & M0.5 & $63 \pm 4$ & 1 & 9.00 & 8.38 & 8.15 & 0.76 \\
\hline J00235732-5531435 & J0023-5531 & $00: 23: 57.32$ & $-55: 31: 43.5$ & M4.1 & $42 \pm 2$ & 3 & 11.11 & 10.55 & 10.24 & 0.16 \\
\hline J00273330-6157169 & J0027-6157 & $00: 27: 33.30$ & $-61: 57: 16.9$ & M4.0 & $44 \pm 2$ & 3 & 10.33 & 9.73 & 9.47 & 0.27 \\
\hline J00284683-6751446 & J0028-6751 & $00: 28: 46.83$ & $-67: 51: 44.6$ & M4.5 & $46 \pm 2$ & 3 & 11.40 & 10.77 & 10.50 & 0.15 \\
\hline J00302572-6236015 & J0030-6236 & $00: 30: 25.72$ & $-62: 36: 01.5$ & M2.2 & $44 \pm 2$ & 3 & 8.44 & 7.80 & 7.55 & 0.54 \\
\hline J00332438-5116433 & J0033-5116 & $00: 33: 24.38$ & $-51: 16: 43.3$ & M3.4 & $42 \pm 2$ & 3 & 9.86 & 9.27 & 9.01 & 0.35 \\
\hline J00421010-5444431 & J0042-5444 & $00: 42: 10.10$ & $-54: 44: 43.1$ & M2.9 & $46 \pm 2$ & 3 & 9.81 & 9.21 & 8.93 & 0.42 \\
\hline J00485254-6526330 & J0048-6526 & $00: 48: 52.54$ & $-65: 26: 33.0$ & M3.2 & $50 \pm 3$ & 3 & 10.41 & 9.85 & 9.55 & 0.31 \\
\hline J00493566-6347416 & J0049-6347 & $00: 49: 35.66$ & $-63: 47: 41.6$ & M1.7 & $46 \pm 2$ & 3 & 9.28 & 8.66 & 8.43 & 0.56 \\
\hline J01024375-6235344 & J0102-6235 & $01: 02: 43.75$ & $-62: 35: 34.4$ & M2.9 & $46 \pm 2$ & 3 & 9.64 & 9.04 & 8.80 & $\ldots$ \\
\hline J01505688-5844032 & J0150-5844 & $01: 50: 56.88$ & $-58: 44: 03.2$ & M3.0 & $46 \pm 2$ & 3 & 9.54 & 8.87 & 8.64 & 0.50 \\
\hline J01521830-5950168 & J0152-5950 & $01: 52: 18.30$ & $-59: 50: 16.8$ & M2-3.0 & $40 \pm 2$ & 2 & 8.94 & 8.33 & 8.14 & 0.57 \\
\hline J02125819-5851182 & J0212-5851 & $02: 12: 58.19$ & $-58: 51: 18.2$ & M1.9 & $48 \pm 2$ & 3 & 9.32 & 8.65 & 8.44 & 0.58 \\
\hline J02205139-5823411 & J0220-5823 & 02:20:51.39 & $-58: 23: 41.1$ & M3.2 & $38 \pm 2$ & 3 & 9.67 & 9.09 & 8.83 & 0.35 \\
\hline J02224418-6022476 & J0222-6022 & $02: 22: 44.18$ & $-60: 22: 47.6$ & M4.0 & $32 \pm 2$ & 2 & 8.99 & 8.39 & 8.10 & 0.43 \\
\hline J02294569-5541496 & J0229-5541 & $02: 29: 45.69$ & $-55: 41: 49.6$ & M4.8 & $46 \pm 2$ & 3 & 11.10 & 10.54 & 10.26 & 0.11 \\
\hline J02414683-5259523 & J0241-5259A & $02: 41: 46.83$ & $-52: 59: 52.3$ & K6.0 & $43 \pm 1^{\mathrm{c}}$ & 2 & 7.58 & 6.93 & 6.76 & 0.73 \\
\hline J02414730-5259306 & J0241-5259B & $02: 41: 47.30$ & $-52: 59: 30.6$ & M2.5 & $44 \pm 3$ & 2 & 8.48 & 7.85 & 7.64 & 0.56 \\
\hline J02423301-5739367 & J0242-5739 & $02: 42: 33.01$ & $-57: 39: 36.7$ & K5.0 & $48 \pm 1^{\mathrm{c}}$ & 2 & 8.56 & 7.97 & 7.78 & 0.73 \\
\hline J02474639-5804272 & J0247-5804 & $02: 47: 46.39$ & $-58: 04: 27.2$ & M1.8 & $44 \pm 2$ & 3 & 9.36 & 8.67 & 8.45 & 0.52 \\
\hline J02543316-5108313 & J0254-5108 & $02: 54: 33.16$ & $-51: 08: 31.3$ & M1.1 & $44 \pm 2$ & 3 & 8.67 & 8.07 & 7.78 & 0.67 \\
\hline $\mathrm{J} 02564708-6343027^{\mathrm{a}}$ & J0256-6343 & 02:56:47.08 & $-63: 43: 02.7$ & M4.0 & $53 \pm 3$ & 1 & 9.86 & 9.22 & 9.01 & 0.49 \\
\hline J02572682-6341293 & J0257-6341 & $02: 57: 26.82$ & $-63: 41: 29.3$ & M3.6 & $63 \pm 3$ & 3 & 10.16 & 9.57 & 9.33 & 0.32 \\
\hline J03114544-4719501 & J0311-4719 & $03: 11: 45.44$ & $-47: 19: 50.1$ & M3.2 & $44 \pm 2$ & 3 & 10.44 & 9.89 & 9.57 & 0.25 \\
\hline J03315564-4359135 & J0331-4359 & $03: 31: 55.64$ & $-43: 59: 13.5$ & K6.0 & $45 \pm 1^{\mathrm{c}}$ & 2 & 8.30 & 7.68 & 7.47 & 0.73 \\
\hline J03512287-5154582 & J0351-5154 & $03: 51: 22.87$ & $-51: 54: 58.2$ & M4.0 & $50 \pm 3$ & 3 & 10.61 & 10.03 & 9.77 & 0.28 \\
\hline J04053964-4014103 & J0405-4014 & 04:05:39.64 & $-40: 14: 10.4$ & M4.2 & $48 \pm 3^{\mathrm{f}}$ & 5 & 9.82 & 9.26 & 8.98 & 0.30 \\
\hline J04074372-6825111 & J0407-6825 & 04:07:43.72 & $-68: 25: 11.1$ & M3.2 & $60 \pm 3$ & 3 & 10.41 & 9.78 & 9.52 & 0.42 \\
\hline J04133609-4413325 & J0413-4413 & $04: 13: 33.14$ & $-52: 31: 58.6$ & M2.4 & $50 \pm 3$ & 3 & 10.00 & 9.35 & 9.12 & 0.42 \\
\hline J04133314-5231586 & J0413-5231 & $04: 13: 36.10$ & $-44: 13: 32.5$ & M3.9 & $60 \pm 3$ & 3 & 10.77 & 10.19 & 9.91 & 0.32 \\
\hline J04440099-6624036 & J0444-6624 & 04:44:00.99 & $-66: 24: 03.6$ & M0.5 & $55 \pm 4$ & 2 & 9.47 & 8.75 & 8.58 & 0.62 \\
\hline J04475779-5035200 & J0447-5035 & $04: 47: 57.79$ & $-50: 35: 20.0$ & M4.0 & $55 \pm 3$ & 3 & 10.06 & 9.43 & 9.21 & 0.28 \\
\hline J04470041-5134405 & J0447-5134 & 04:47:00.41 & $-51: 34: 40.5$ & M1.9 & $60 \pm 3$ & 3 & 10.87 & 10.30 & 10.02 & 0.30 \\
\hline J05332558-5117131 & J0533-5117 & $05: 33: 25.58$ & $-51: 17: 13.1$ & K7.0 & $55 \pm 1^{\mathrm{c}}$ & 2 & 8.99 & 8.36 & 8.16 & 0.63 \\
\hline J17080882-6936186 & J1708-6936 & 17:08:08.82 & $-69: 36: 18.6$ & M3.5 & $49 \pm 3$ & 2 & 9.06 & 8.42 & 8.20 & 0.54 \\
\hline J19225071-6310581 & J1922-6310 & $19: 22: 50.71$ & $-63: 10: 58.1$ & M3.0 & $61 \pm 4$ & 2 & 9.45 & 8.82 & 8.58 & 0.66 \\
\hline J20423672-5425263 & $\mathrm{J} 2042-5425$ & $20: 42: 36.72$ & $-54: 25: 26.3$ & M4.0 & $48 \pm 2$ & 3 & 10.75 & 10.16 & 9.86 & 0.24 \\
\hline J21083826-4244540 & J2108-4244 & $21: 08: 38.26$ & $-42: 44: 54.0$ & M4.4 & $44 \pm 2$ & 3 & 10.14 & 9.57 & 9.24 & 0.20 \\
\hline J21100614-5811483 & $\mathrm{J} 2110-5811$ & 21:10:06.14 & $-58: 11: 48.3$ & M4.0 & $52 \pm 3$ & 3 & 10.89 & 10.33 & 10.07 & 0.24 \\
\hline J21143354-4213528 & J2114-4213 & $21: 14: 33.54$ & $-42: 13: 52.8$ & M3.9 & $52 \pm 3$ & 3 & 11.38 & 10.78 & 10.53 & 0.18 \\
\hline $\mathrm{J} 21163528-6005124$ & $\mathrm{~J} 2116-6005$ & $21: 16: 35.28$ & $-60: 05: 12.4$ & M3.5 & $48 \pm 2$ & 3 & 10.19 & 9.56 & 9.31 & 0.35 \\
\hline J21354554-4218343 & $\mathrm{J} 2135-4218$ & $21: 35: 45.54$ & $-42: 18: 34.3$ & M5.2 & $58 \pm 3$ & 3 & 11.68 & 11.15 & 10.81 & 0.17 \\
\hline J21490499-6413039 & $\mathrm{J} 2149-6413$ & 21:49:04.99 & $-64: 13: 03.9$ & M4.5 & $44 \pm 2$ & 2 & 10.35 & 9.80 & 9.47 & 0.18 \\
\hline J22025453-6440441 & $\mathrm{J} 2202-6440$ & $22: 02: 54.53$ & $-64: 40: 44.1$ & M1.8 & $46 \pm 2$ & 3 & 9.06 & 8.41 & 8.16 & 0.62 \\
\hline J22440873-5413183 & $\mathrm{J} 2244-5413$ & $22: 44: 08.73$ & $-54: 13: 18.3$ & M4.0 & $49 \pm 4$ & 2 & 9.36 & 8.71 & 8.47 & 0.42 \\
\hline J22463471-7353504 & $\mathrm{J} 2246-7353$ & $22: 46: 34.71$ & $-73: 53: 50.4$ & M2.3 & $52 \pm 3$ & 3 & 9.66 & 9.05 & 8.81 & 0.54 \\
\hline $\mathrm{J} 22470872-6920447^{\mathrm{a}}$ & $\mathrm{J} 2247-6920$ & $22: 47: 08.72$ & $-69: 20: 44.7$ & K6.0 & $52 \pm 1^{\mathrm{c}}$ & 1 & 8.89 & 8.30 & 8.09 & 0.68 \\
\hline J23474694-6517249 & J2347-6517 & $23: 47: 46.94$ & $-65: 17: 24.9$ & M1.5 & $45 \pm 2$ & 2 & 9.10 & 8.39 & 8.17 & 0.61 \\
\hline \multicolumn{11}{|c|}{ Columba (Col) } \\
\hline J01424689-5126469 & $\mathrm{J} 0142-5126$ & $01: 42: 46.89$ & $-51: 26: 46.9$ & M6.5 & $66 \pm 6^{\mathrm{f}}$ & 5 & 11.08 & 10.58 & 10.10 & 0.21 \\
\hline $\mathrm{J} 02365171-5203036^{\mathrm{a}}$ & J0236-5203 & $02: 36: 51.71$ & $-52: 03: 03.6$ & M2.0 & $39 \pm 2$ & 1 & 8.42 & 7.76 & 7.50 & 0.68 \\
\hline J03241504-5901125 & J0324-5901 & $03: 24: 15.04$ & $-59: 01: 12.5$ & K7.0 & $90 \pm 5$ & 2 & 9.55 & 8.92 & 8.72 & 0.78 \\
\hline J03320347-5139550 & J0332-5139 & 03:32:03.47 & $-51: 39: 55.0$ & M2.0 & $88 \pm 5$ & 1 & 10.23 & 9.58 & 9.35 & 0.62 \\
\hline
\end{tabular}


Table 3

(Continued)

\begin{tabular}{|c|c|c|c|c|c|c|c|c|c|c|}
\hline $\begin{array}{l}\text { Target } \\
\text { 2MASS ID }\end{array}$ & $\begin{array}{l}\text { Abbrv. } \\
\text { Name }\end{array}$ & R.A. & Decl. & SpT & $\begin{array}{c}\text { Distance }^{\mathrm{b}} \\
\quad(\mathrm{pc})\end{array}$ & References & $J$ & $H$ & $K$ & $\begin{array}{c}M_{\text {prim }}{ }^{\mathrm{g}} \\
\left(M_{\odot}\right)\end{array}$ \\
\hline J03494535-6730350 & J0349-6730 & $03: 49: 45.35$ & $-67: 30: 35.0$ & $\mathrm{~K} 7.0$ & $81 \pm 4$ & 2 & 9.85 & 9.23 & 9.03 & 0.70 \\
\hline J04091413-4008019 & J0409-4008 & 04:09:14.13 & $-40: 08: 01.9$ & M3.5 & $63 \pm 5$ & 2 & 10.65 & 10.00 & 9.77 & 0.38 \\
\hline J04240094-5512223 & J0424-5512 & 04:24:00.94 & $-55: 12: 22.3$ & M2.5 & $68 \pm 5$ & 2 & 9.80 & 9.16 & 8.95 & 0.64 \\
\hline J04515303-4647309 & J0451-4647 & $04: 51: 53.03$ & $-46: 47: 30.9$ & M0.0 & $76 \pm 6$ & 2 & 9.80 & 9.14 & 8.89 & 0.59 \\
\hline J05111098-4903597 & J0511-4903 & $05: 11: 10.98$ & $-49: 03: 59.7$ & M3.5 & $62 \pm 6$ & 2 & 10.64 & 10.01 & 9.77 & 0.38 \\
\hline J05164586-5410168 & J0516-5410 & $05: 16: 45.86$ & $-54: 10: 16.8$ & M3.0 & $69 \pm 6$ & 2 & 10.43 & 9.78 & 9.55 & 0.50 \\
\hline J05392505-4245211 & J0539-4245 & 05:39:25.05 & $-42: 45: 21.1$ & M2.0 & $41 \pm 4$ & 1 & 9.45 & 8.80 & 8.60 & 0.45 \\
\hline J07065772-5353463 & J0706-5353 & 07:06:57.72 & $-53: 53: 46.3$ & M0.0 & $53 \pm 5$ & 2 & 8.54 & 7.90 & 7.67 & 0.78 \\
\hline $\mathrm{J} 07170438-6311123^{\mathrm{a}}$ & J0717-6311 & $07: 17: 04.38$ & $-63: 11: 12.3$ & M2.0 & $58 \pm 4$ & 1 & 9.73 & 9.09 & 8.86 & 0.39 \\
\hline J08152160-4918303 & J0815-4918 & $08: 15: 21.60$ & $-49: 18: 30.3$ & G7.0 & $120 \pm 4^{\mathrm{c}}$ & 4 & 9.61 & 9.19 & 9.11 & 0.89 \\
\hline J09331427-4848331 & J0933-4848 & $09: 33: 14.27$ & $-48: 48: 33.1$ & $\mathrm{~K} 7.0$ & $46 \pm 1^{c}$ & 2 & 8.94 & 8.33 & 8.10 & 0.63 \\
\hline \multicolumn{11}{|c|}{$\beta$ Pictoris (bPic) } \\
\hline J00172353-6645124 & J0017-6645 & $00: 17: 24$ & $-66: 45: 12.4$ & M2.5 & $39 \pm 2$ & 2 & 8.56 & 7.93 & 7.70 & 0.60 \\
\hline J05332802-4257205 & J0533-4257 & $05: 33: 28.02$ & $-42: 57: 20.5$ & M4.5 & $16 \pm 4$ & 1 & 8.00 & 7.40 & 7.12 & 0.17 \\
\hline J08475676-7854532 & J0847-7854 & $08: 47: 56.76$ & $-78: 54: 53.2$ & M3.0 & $66 \pm 7$ & 1 & 9.32 & 8.68 & 8.41 & 0.72 \\
\hline J11493184-7851011 & $\mathrm{J} 1149-7851$ & $11: 49: 31.84$ & $-78: 51: 01.1$ & M1.0 & $68 \pm 6$ & 2 & 9.45 & 8.72 & 8.49 & 0.72 \\
\hline J13545390-7121476 & $\mathrm{J} 1354-7121$ & $13: 54: 53.90$ & $-71: 21: 47.6$ & M2.5 & $21 \pm 1$ & 2 & 8.55 & 7.92 & 7.67 & 0.25 \\
\hline $\mathrm{J} 14252913-4113323^{\mathrm{a}}$ & $\mathrm{J} 1425-4113$ & $14: 25: 29.13$ & $-41: 13: 32.3$ & M2.5 & $66 \pm 4$ & 2 & 8.55 & 7.91 & 7.61 & 0.73 \\
\hline J16572029-5343316 & $\mathrm{J} 1657-5343$ & $16: 57: 20.29$ & $-53: 43: 31.6$ & M3.0 & $51 \pm 3$ & 2 & 8.69 & 8.07 & 7.79 & 0.53 \\
\hline J17292067-5014529 & J1729-5014 & $17: 29: 20.67$ & $-50: 14: 52.9$ & M3.0 & $64 \pm 5$ & 2 & 8.87 & 8.19 & 7.99 & 0.64 \\
\hline $\mathrm{J} 18142207-3246100$ & $\mathrm{~J} 1814-3246$ & $18: 14: 22.07$ & $-32: 46: 10.0$ & M1.5 & $90 \pm 8$ & 2 & 9.44 & 8.77 & 8.54 & 0.87 \\
\hline J18420694-5554254 & $\mathrm{J} 1842-5554$ & $18: 42: 06.94$ & $-55: 54: 25.4$ & M3.5 & $54 \pm 4$ & 2 & 9.49 & 8.82 & 8.58 & 0.54 \\
\hline J18465255-6210366 & $\mathrm{J} 1846-6210$ & $18: 46: 52.55$ & $-62: 10: 36.6$ & M1.0 & $54 \pm 3$ & 2 & 8.75 & 8.05 & 7.85 & 0.76 \\
\hline J19233820-4606316 & J1923-4606 & $19: 23: 38.20$ & $-46: 06: 31.6$ & M0.0 & $70 \pm 4$ & 2 & 9.11 & 8.44 & 8.27 & 0.81 \\
\hline J21212873-6655063 & $\mathrm{J} 2121-6655$ & $21: 21: 28.73$ & $-66: 55: 06.3$ & K7.0 & $32 \pm 1^{\mathrm{c}}$ & 2 & 7.88 & 7.26 & 7.01 & 0.55 \\
\hline \multicolumn{11}{|c|}{ Argus (Arg) } \\
\hline J04464970-6034109 & J0446-6034 & $04: 46: 49.70$ & $-60: 34: 10.9$ & M1.5 & $37 \pm 2$ & 2 & 8.55 & 7.95 & 7.72 & 0.62 \\
\hline J05090356-4209199 & J0509-4209 & 05:09:03.56 & $-42: 09: 19.9$ & M3.5 & $51 \pm 4$ & 2 & 9.58 & 8.98 & 8.76 & 0.54 \\
\hline J06380031-4056011 & J0638-4056 & $06: 38: 00.31$ & $-40: 56: 01.1$ & M3.5 & $35 \pm 2$ & 1 & 10.35 & 9.81 & 9.53 & 0.19 \\
\hline J10252563-4918389 & $\mathrm{J} 1025-4918$ & $10: 25: 25.63$ & $-49: 18: 38.9$ & M4.0 & $27 \pm 1$ & 2 & 9.12 & 8.51 & 8.26 & 0.30 \\
\hline J11234697-5257393 & $\mathrm{J} 1123-5257$ & $11: 23: 46.97$ & $-52: 57: 39.3$ & Mo.0 & $49 \pm 1^{\mathrm{c}}$ & 4 & 8.79 & 8.20 & 7.95 & 0.70 \\
\hline J12233860-4606203 & $\mathrm{J} 1223-4606$ & $12: 23: 38.60$ & $-46: 06: 20.3$ & M4.0 & $23 \pm 1$ & 1 & 9.53 & 8.94 & 8.70 & 0.18 \\
\hline J14284804-7430205 & $\mathrm{J} 1428-7430$ & $14: 28: 48.04$ & $-74: 30: 20.5$ & M1.0 & $76 \pm 3$ & 1 & 9.26 & 8.57 & 8.35 & 0.80 \\
\hline J15163224-5855237 & $\mathrm{J} 1516-5855$ & $15: 16: 32.24$ & $-58: 55: 23.7$ & K7.0 & $77 \pm 3$ & 1 & 9.10 & 8.55 & 8.29 & 0.67 \\
\hline $\mathrm{J} 20072376-5147272^{\mathrm{a}}$ & J2007-5147 & 20:07:23.76 & $-51: 47: 27.2$ & K6.0 & $34 \pm 0^{\mathrm{c}}$ & 1 & 8.16 & 7.57 & 7.39 & 0.66 \\
\hline $\mathrm{J} 23532520-7056410$ & $\mathrm{~J} 2353-7056$ & $23: 53: 25.20$ & $-70: 56: 41.0$ & M3.5 & $17 \pm 1$ & 2 & 8.68 & 8.10 & 7.78 & 0.21 \\
\hline \multicolumn{11}{|c|}{ Carina (Car) } \\
\hline J07540718-6320149 & J0754-6320 & 07:54:07.18 & $-63: 20: 14.9$ & M3.0 & $80 \pm 5$ & 2 & 10.33 & 9.69 & 9.45 & 0.45 \\
\hline J08094269-5652199 & J0809-5652 & 08:09:42.69 & $-56: 52: 19.9$ & K0.0 & $104 \pm 3^{\mathrm{c}}$ & 4 & 9.38 & 8.98 & 8.83 & 0.90 \\
\hline J08185942-7239561 & J0818-7239 & $08: 18: 59.42$ & $-72: 39: 56.1$ & M0.0 & $60 \pm 2$ & 1 & 9.78 & 9.15 & 8.94 & 0.42 \\
\hline J09032434-6348330 & J0903-6348 & $09: 03: 24.34$ & $-63: 48: 33.0$ & M0.5 & $66 \pm 3$ & 2 & 9.57 & 8.86 & 8.69 & 0.67 \\
\hline J09111581-5014149 & J0911-5014 & 09:11:15.81 & $-50: 14: 14.9$ & K5.0 & $117 \pm 5^{\mathrm{c}}$ & 4 & 10.23 & 9.65 & 9.50 & 0.77 \\
\hline J09312541-5314366 & J0931-5314 & $09: 31: 25.41$ & $-53: 14: 36.6$ & K5.0 & $120 \pm 19$ & 4 & 10.19 & 9.66 & 9.50 & 0.76 \\
\hline \multicolumn{11}{|c|}{ AB Doradus (ABDor) } \\
\hline J01484087-4830519 & J0148-4830 & $01: 48: 40.87$ & $-48: 30: 51.9$ & M1.5 & $36 \pm 2$ & 2 & 9.19 & 8.55 & 8.36 & 0.53 \\
\hline J05240991-4223054 & J0524-4223 & 05:24:09.91 & $-42: 23: 05.4$ & M0.5 & $52 \pm 9$ & 2 & 10.58 & 9.92 & 9.72 & 0.37 \\
\hline J05381615-6923321 ${ }^{\mathrm{a}}$ & J0538-6923 & $05: 38: 16.15$ & $-69: 23: 32.1$ & M0.5 & $21 \pm 2$ & 1 & 8.96 & 8.29 & 8.11 & 0.37 \\
\hline J05531299-4505119 & J0553-4505 & 05:53:12.99 & $-45: 05: 11.9$ & M0.5 & $34 \pm 4$ & 2 & 8.60 & 7.94 & 7.73 & 0.61 \\
\hline J08465879-7246588 & J0846-7246 & $08: 46: 58.79$ & $-72: 46: 58.8$ & $\mathrm{~K} 7.0$ & $45 \pm 1^{\mathrm{c}}$ & 1 & 8.49 & 7.81 & 7.60 & 0.61 \\
\hline J15244849-4929473 & J1524-4929 & $15: 24: 48.49$ & $-49: 29: 47.3$ & M2.0 & $23 \pm 1$ & 2 & 8.16 & 7.53 & 7.30 & 0.54 \\
\hline \multicolumn{11}{|c|}{ TW Hydrae (TWA) } \\
\hline $\mathrm{J} 11455177-5520456^{\mathrm{a}}$ & $\mathrm{J} 1145-5520$ & $11: 45: 51.77$ & $-55: 20: 45.6$ & K5.0 & $43 \pm 1^{c}$ & 1 & 8.02 & 7.41 & 7.27 & 0.63 \\
\hline J12313807-4558593 & $\mathrm{J} 1231-4558$ & $12: 31: 38.07$ & $-45: 58: 59.3$ & M3.0 & $78 \pm 3$ & 2 & 9.33 & 8.69 & 8.41 & 0.39 \\
\hline
\end{tabular}


Table 3

(Continued)

\begin{tabular}{|c|c|c|c|c|c|c|c|c|c|c|}
\hline $\begin{array}{l}\text { Target } \\
\text { 2MASS ID }\end{array}$ & $\begin{array}{l}\text { Abbrv. } \\
\text { Name }\end{array}$ & R.A. & Decl. & $\mathrm{SpT}$ & $\begin{array}{c}\text { Distance }^{b} \\
(\mathrm{pc})\end{array}$ & References & $J$ & $H$ & $K$ & $\begin{array}{c}M_{\text {prim }}{ }^{\mathrm{g}} \\
\left(M_{\odot}\right)\end{array}$ \\
\hline J12345629-4538075 & J1234-4538 & $12: 34: 56.29$ & $-45: 38: 07.5$ & M1.5 & $78 \pm 3^{\mathrm{e}}$ & 2 & 8.99 & 8.33 & 8.09 & 0.49 \\
\hline J12350424-4136385 & J1235-4136 & $12: 35: 04.24$ & $-41: 36: 38.5$ & M2.0 & $59 \pm 2^{\mathrm{d}}$ & 2 & 9.12 & 8.48 & 8.19 & 0.50 \\
\hline
\end{tabular}

Notes.

a Denotes that the YMG designation is ambiguous according to Malo et al. $(2013,2014)$.

${ }^{\mathrm{b}}$ The distance estimate for each target is either statistical/kinematical or from trigonometric parallax, taken from their respective references unless ${ }^{\mathrm{c}}$, ${ }^{\mathrm{d}}$, or ${ }^{\mathrm{e}}$.

c Gaia Collaboration (2016).

d Donaldson et al. (2016).

e Weinberger et al. (2013).

${ }^{\mathrm{f}}$ No distance error is reported in the source reference. The error estimate is based on the average error for other targets in this YMG.

${ }^{g}$ Mass calculations are discussed in Sections 4.1 and 5.2.

References. 1. Malo et al. (2013), 2. Malo et al. (2014), 3. Kraus et al. (2014), 4. Moór et al. (2013), 5. Rodriguez et al. (2013).

(This table is available in machine-readable form.)

\section{Data}

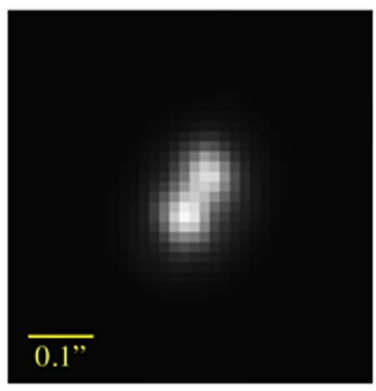

Model

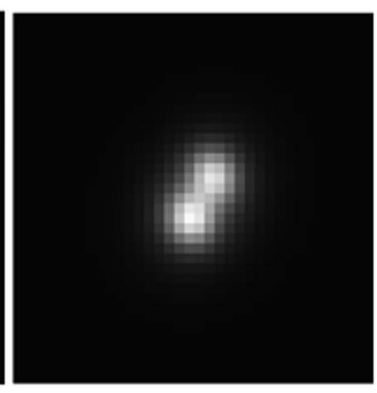

Residuals

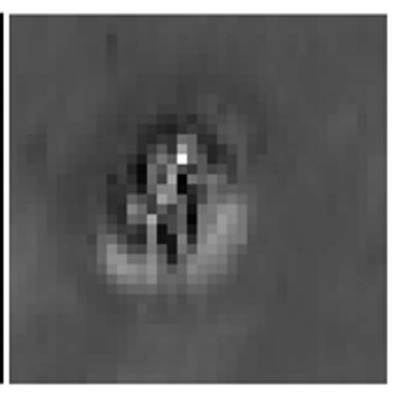

Figure 2. Example of a PSF-fit to a binary (J0717-6311), separation $=0$ ". $08, \Delta H=0.09$. Left: pre-processed data image. Middle: best-fit triple bivariate Gaussian model. Right: fit residuals. The stretch on the residuals plot is $1 / 10$ that of the data and model plots.

We use Clio's narrow camera mode (according to the measurements of Morzinski et al. 2015: plate scale $=15.846$ $\pm 0.064 \mathrm{mas} / \mathrm{pixel}$; instrument north angle $=-1.8 \pm 0.34$, FOV $=16 \times 8^{\prime \prime}$ ) and conducted most of our observations through the $\mathrm{H}$ bandpass, except in one run, where the Ks filter was used. These filters are only slightly offset from their 2MASS counterparts (see Males et al. 2014, Tables 7 and 8 therein), hence throughout this paper we use Clio photometry interchangeably with 2MASS. For each target, one or two sets of four images were taken in an ABBA nodding scheme. Each image is composed of 20 coadded exposures, each $280 \mathrm{~ms}$ long, which prevents saturation. A summary of the run dates and filters used is presented in Table 2.

One hundred and five targets were observed over five epochs, 30 of which have been imaged in multiple epochs. Table 3 lists the observed targets and their relevant properties. A distribution of their spectral types as listed in their source references may be found in Figure 1. They are chiefly pre-main sequence $\mathrm{K}$ and $\mathrm{M}$ dwarfs. We have also imaged a late-G- and an early-K member in our program due to a paucity of other viable targets at the times when the observations were taken. In Section 5 we explicitly divide the analysis by spectral type and mass.

Fourteen of our YMG targets have Tycho designations and are part of the recent Gaia Data Release 1 (Gaia Collaboration 2016). For these targets, we adopt the Gaia distances, which are consistent with the statistical distances derived from their sources in the literature. In addition to the targets in Table 3, we observed J06511418-4037510, J12170465-5743558, J10252092-4241539, and J13213722-4421518, which were later eliminated from our sample because of new distance information. The first two have Gaia parallax measurements consistent with being background giants and are thus excluded from analysis. This means that $10 \%-20 \%$ of the sample may have incorrect YMG designations. The last two have been rejected by Donaldson et al. (2016) from membership in TWA, also on the basis of parallax data. None of these four objects harbors obvious visual companions down to 0 ". 08 . Future Gaia releases will clarify the YMG assignments for the remaining targets.

\subsection{Data Reduction}

Sky subtraction. We perform sky subtraction by directly subtracting the nod pairs that are temporally closest (i.e., "A" and " $\mathrm{B}$ " images). This procedure also eliminates the dark and bias current.

Bad pixels. We exclude bad pixels and obvious cosmic rays from any analysis and source-fitting we perform when extracting measurements from our images. This is done by omitting these pixels from calculations of goodness-of-fit metrics.

Flat fielding. H-band flat fields are constructed from MagAO twilight flats (and darks) taken on the same night as the 
Table 4

Target HWHMs

\begin{tabular}{|c|c|c|c|c|c|c|c|}
\hline $\begin{array}{l}\text { Target } \\
\text { Name }\end{array}$ & $\begin{array}{c}\text { \# of } \\
\text { Epochs }\end{array}$ & $\begin{array}{l}\text { Best } \\
\text { Epoch }\end{array}$ & Band & $\begin{array}{c}\text { Minor } \\
\text { Axis (") }\end{array}$ & $\begin{array}{l}\text { Axis } \\
\text { Ratio }\end{array}$ & $\begin{array}{c}\text { Orientation } \\
\text { Angle }\left({ }^{\circ}\right)\end{array}$ & Multiple? $^{a}$ \\
\hline J0012-7952 & 1 & $14-11-10$ & $\mathrm{Ks}$ & $0.038 \pm 0.001$ & $0.88 \pm 0.02$ & $34 \pm 6$ & $\mathrm{~N}$ \\
\hline J0014-6003 & 1 & $14-11-10$ & Ks & $0.039 \pm 0.002$ & $0.91 \pm 0.03$ & $4 \pm 6$ & $\mathrm{~N}$ \\
\hline J0015-6414 & 1 & $14-11-10$ & Ks & $0.039 \pm 0.008$ & $0.83 \pm 0.06$ & $4 \pm 12$ & $\mathrm{~N}$ \\
\hline J0017-6645 & 1 & $14-11-12$ & Ks & $0.034 \pm 0.000$ & $0.88 \pm 0.01$ & $35 \pm 7$ & $\mathrm{~N}$ \\
\hline J0017-7032 & 1 & $14-11-10$ & $\mathrm{Ks}$ & $0.041 \pm 0.001$ & $0.90 \pm 0.03$ & $175 \pm 7$ & $\mathrm{Y}$ \\
\hline J0023-5531 & 1 & $14-11-10$ & Ks & $0.043 \pm 0.001$ & $0.84 \pm 0.01$ & $83 \pm 7$ & $\mathrm{~N}$ \\
\hline J0027-6157 & 1 & $14-11-10$ & Ks & $0.042 \pm 0.000$ & $0.87 \pm 0.02$ & $92 \pm 6$ & $\mathrm{~N}$ \\
\hline J0028-6751 & 1 & $14-11-10$ & $\mathrm{Ks}$ & $0.055 \pm 0.003$ & $0.86 \pm 0.03$ & $111 \pm 8$ & $\mathrm{~N}$ \\
\hline J0030-6236 & 1 & $15-11-26$ & $\mathrm{H}$ & $0.045 \pm 0.007$ & $0.88 \pm 0.02$ & $32 \pm 14$ & $\mathrm{Y}$ \\
\hline J0033-5116 & 1 & $14-11-12$ & Ks & $0.037 \pm 0.000$ & $0.86 \pm 0.04$ & $49 \pm 8$ & $\mathrm{~N}$ \\
\hline J0042-5444 & 1 & $14-11-12$ & Ks & $0.038 \pm 0.001$ & $0.90 \pm 0.02$ & $72 \pm 7$ & $\mathrm{~N}$ \\
\hline J0048-6526 & 1 & $14-12-01$ & $\mathrm{H}$ & $0.077 \pm 0.002$ & $0.93 \pm 0.02$ & $127 \pm 13$ & $\mathrm{~N}$ \\
\hline J0049-6347 & 1 & $14-12-01$ & $\mathrm{H}$ & $0.037 \pm 0.003$ & $0.92 \pm 0.03$ & $30 \pm 19$ & $\mathrm{~N}$ \\
\hline J0102-6235 & 2 & $15-11-26$ & $\mathrm{H}$ & $0.113 \pm 0.016$ & $0.76 \pm 0.04$ & $160 \pm 5$ & $\mathrm{Y}$ \\
\hline J0142-5126 & 1 & $15-11-26$ & $\mathrm{H}$ & $0.212 \pm 0.041$ & $0.89 \pm 0.05$ & $44 \pm 24$ & $\mathrm{Y}$ \\
\hline J0148-4830 & 3 & $14-11-30$ & $\mathrm{H}$ & $0.060 \pm 0.019$ & $0.72 \pm 0.04$ & $152 \pm 8$ & $\mathrm{~N}$ \\
\hline J0150-5844 & 1 & $14-11-30$ & $\mathrm{H}$ & $0.046 \pm 0.007$ & $0.93 \pm 0.05$ & $145 \pm 15$ & $\mathrm{~N}$ \\
\hline J0152-5950 & 1 & $14-11-30$ & $\mathrm{H}$ & $0.030 \pm 0.001$ & $0.84 \pm 0.03$ & $35 \pm 6$ & $\mathrm{~N}$ \\
\hline J0212-5851 & 2 & $14-11-30$ & $\mathrm{H}$ & $0.032 \pm 0.001$ & $0.84 \pm 0.02$ & $34 \pm 9$ & $\mathrm{~N}$ \\
\hline J0220-5823 & 1 & $14-11-30$ & $\mathrm{H}$ & $0.037 \pm 0.002$ & $0.88 \pm 0.07$ & $49 \pm 26$ & $\mathrm{~N}$ \\
\hline J0222-6022 & 1 & $14-11-30$ & $\mathrm{H}$ & $0.032 \pm 0.001$ & $0.84 \pm 0.04$ & $47 \pm 11$ & $\mathrm{Y}$ \\
\hline J0229-5541 & 1 & $14-11-30$ & $\mathrm{H}$ & $0.071 \pm 0.004$ & $0.89 \pm 0.05$ & $135 \pm 6$ & $\mathrm{Y}$ \\
\hline J0236-5203 & 1 & $14-12-01$ & $\mathrm{H}$ & $0.056 \pm 0.010$ & $0.92 \pm 0.05$ & $143 \pm 14$ & $\mathrm{~N}$ \\
\hline J0241-5259A & 2 & $14-12-01$ & $\mathrm{H}$ & $0.050 \pm 0.010$ & $0.79 \pm 0.09$ & $129 \pm 24$ & $\mathrm{Y}$ \\
\hline J0241-5259B & 1 & $14-12-01$ & $\mathrm{H}$ & $0.043 \pm 0.003$ & $0.91 \pm 0.04$ & $26 \pm 15$ & $\mathrm{Y}$ \\
\hline J0242-5739 & 1 & $14-12-01$ & $\mathrm{H}$ & $0.032 \pm 0.001$ & $0.87 \pm 0.01$ & $31 \pm 8$ & $\mathrm{~N}$ \\
\hline J0247-5804 & 1 & $14-12-01$ & $\mathrm{H}$ & $0.053 \pm 0.002$ & $0.89 \pm 0.01$ & $121 \pm 5$ & $\mathrm{~N}$ \\
\hline J0254-5108 & 1 & $14-12-01$ & $\mathrm{H}$ & $0.033 \pm 0.003$ & $0.87 \pm 0.02$ & $40 \pm 13$ & $\mathrm{~N}$ \\
\hline J0256-6343 & 1 & $14-12-01$ & $\mathrm{H}$ & $0.055 \pm 0.003$ & $0.74 \pm 0.03$ & $151 \pm 3$ & $\mathrm{~N}$ \\
\hline J0257-6341 & 1 & $14-12-01$ & $\mathrm{H}$ & $0.058 \pm 0.004$ & $0.83 \pm 0.06$ & $126 \pm 6$ & $\mathrm{Y}$ \\
\hline J0311-4719 & 1 & $14-11-10$ & Ks & $0.042 \pm 0.001$ & $0.85 \pm 0.02$ & $144 \pm 3$ & $\mathrm{~N}$ \\
\hline J0324-5901 & 1 & $14-11-30$ & $\mathrm{H}$ & $0.048 \pm 0.021$ & $0.82 \pm 0.05$ & $22 \pm 10$ & $\mathrm{Y}$ \\
\hline J0331-4359 & 1 & $14-11-30$ & $\mathrm{H}$ & $0.028 \pm 0.001$ & $0.84 \pm 0.01$ & $30 \pm 4$ & $\mathrm{Y}$ \\
\hline J0332-5139 & 1 & $14-11-30$ & $\mathrm{H}$ & $0.034 \pm 0.002$ & $0.90 \pm 0.02$ & $44 \pm 13$ & $\mathrm{Y}$ \\
\hline J0349-6730 & 1 & $14-11-30$ & $\mathrm{H}$ & $0.032 \pm 0.001$ & $0.84 \pm 0.02$ & $26 \pm 5$ & $\mathrm{~N}$ \\
\hline J0351-5154 & 1 & $14-11-10$ & Ks & $0.044 \pm 0.001$ & $0.92 \pm 0.01$ & $154 \pm 4$ & $\mathrm{~N}$ \\
\hline J0405-4014 & 1 & $15-11-27$ & $\mathrm{H}$ & $0.042 \pm 0.001$ & $0.95 \pm 0.03$ & $142 \pm 4$ & $\mathrm{Y}$ \\
\hline J0407-6825 & 1 & $14-11-10$ & $\mathrm{Ks}$ & $0.039 \pm 0.000$ & $0.86 \pm 0.02$ & $70 \pm 3$ & $\mathrm{~N}$ \\
\hline J0409-4008 & 1 & $14-12-01$ & $\mathrm{H}$ & $0.040 \pm 0.001$ & $0.87 \pm 0.02$ & $87 \pm 3$ & $\mathrm{~N}$ \\
\hline J0413-4413 & 1 & $14-11-10$ & Ks & $0.043 \pm 0.002$ & $0.89 \pm 0.02$ & $138 \pm 17$ & $\mathrm{~N}$ \\
\hline J0413-5231 & 1 & $14-11-10$ & $\mathrm{Ks}$ & $0.039 \pm 0.001$ & $0.90 \pm 0.02$ & $115 \pm 10$ & $\mathrm{~N}$ \\
\hline J0424-5512 & 1 & $14-12-01$ & $\mathrm{H}$ & $0.042 \pm 0.002$ & $0.88 \pm 0.11$ & $57 \pm 41$ & $\mathrm{~N}$ \\
\hline J0444-6624 & 1 & $14-11-30$ & $\mathrm{H}$ & $0.044 \pm 0.014$ & $0.80 \pm 0.01$ & $29 \pm 10$ & $\mathrm{~N}$ \\
\hline J0446-6034 & 1 & $14-11-30$ & $\mathrm{H}$ & $0.032 \pm 0.002$ & $0.76 \pm 0.03$ & $42 \pm 4$ & $\mathrm{~N}$ \\
\hline J0447-5035 & 1 & $14-12-01$ & $\mathrm{H}$ & $0.057 \pm 0.003$ & $0.88 \pm 0.02$ & $121 \pm 5$ & $\mathrm{Y}$ \\
\hline J0447-5134 & 1 & $14-11-30$ & $\mathrm{H}$ & $0.037 \pm 0.002$ & $0.87 \pm 0.05$ & $110 \pm 9$ & $\mathrm{~N}$ \\
\hline J0451-4647 & 1 & $14-11-30$ & $\mathrm{H}$ & $0.036 \pm 0.002$ & $0.81 \pm 0.01$ & $106 \pm 12$ & $\mathrm{Y}$ \\
\hline J0509-4209 & 1 & $14-11-30$ & $\mathrm{H}$ & $0.039 \pm 0.001$ & $0.86 \pm 0.03$ & $112 \pm 9$ & $\mathrm{~N}$ \\
\hline J0511-4903 & 3 & $14-12-01$ & $\mathrm{H}$ & $0.061 \pm 0.003$ & $0.85 \pm 0.03$ & $120 \pm 2$ & $\mathrm{~N}$ \\
\hline J0516-5410 & 1 & $14-12-01$ & $\mathrm{H}$ & $0.050 \pm 0.004$ & $0.89 \pm 0.03$ & $82 \pm 9$ & $\mathrm{~N}$ \\
\hline J0524-4223 & 1 & $14-12-01$ & $\mathrm{H}$ & $0.041 \pm 0.001$ & $0.88 \pm 0.03$ & $88 \pm 9$ & $\mathrm{Y}$ \\
\hline J0533-4257 & 2 & $14-12-01$ & $\mathrm{H}$ & $0.043 \pm 0.008$ & $0.83 \pm 0.08$ & $5 \pm 31$ & $\mathrm{Y}$ \\
\hline J0533-5117 & 3 & $14-12-01$ & $\mathrm{H}$ & $0.032 \pm 0.001$ & $0.83 \pm 0.04$ & $29 \pm 3$ & $\mathrm{Y}$ \\
\hline J0538-6923 & 1 & $14-12-01$ & $\mathrm{H}$ & $0.058 \pm 0.009$ & $0.88 \pm 0.06$ & $83 \pm 8$ & $\mathrm{~N}$ \\
\hline J0539-4245 & 1 & $14-12-01$ & $\mathrm{H}$ & $0.047 \pm 0.005$ & $0.86 \pm 0.08$ & $57 \pm 24$ & $\mathrm{~N}$ \\
\hline J0553-4505 & 1 & $14-11-30$ & $\mathrm{H}$ & $0.041 \pm 0.009$ & $0.73 \pm 0.15$ & $63 \pm 24$ & $\mathrm{~N}$ \\
\hline J0638-4056 & 2 & $14-12-01$ & $\mathrm{H}$ & $0.077 \pm 0.005$ & $0.90 \pm 0.09$ & $41 \pm 2$ & $\mathrm{~N}$ \\
\hline J0706-5353 & 1 & $14-04-21$ & $\mathrm{H}$ & $0.028 \pm 0.002$ & $0.79 \pm 0.06$ & $57 \pm 5$ & $\mathrm{~N}$ \\
\hline J0717-6311 & 2 & $14-04-21$ & $\mathrm{H}$ & $0.037 \pm 0.000$ & $0.89 \pm 0.01$ & $68 \pm 5$ & $\mathrm{Y}$ \\
\hline J0754-6320 & 2 & $14-04-21$ & $\mathrm{H}$ & $0.050 \pm 0.002$ & $0.84 \pm 0.07$ & $140 \pm 5$ & $\mathrm{Y}$ \\
\hline J0809-5652 & 2 & $14-12-01$ & $\mathrm{H}$ & $0.032 \pm 0.002$ & $0.84 \pm 0.01$ & $37 \pm 5$ & $\mathrm{Y}$ \\
\hline J0815-4918 & 1 & $14-12-01$ & $\mathrm{H}$ & $0.035 \pm 0.005$ & $0.88 \pm 0.05$ & $33 \pm 21$ & $\mathrm{~N}$ \\
\hline J0818-7239 & 3 & $14-04-21$ & $\mathrm{H}$ & $0.032 \pm 0.001$ & $0.80 \pm 0.02$ & $32 \pm 4$ & $\mathrm{Y}$ \\
\hline
\end{tabular}


Table 4

(Continued)

\begin{tabular}{|c|c|c|c|c|c|c|c|}
\hline $\begin{array}{l}\text { Target } \\
\text { Name }\end{array}$ & $\begin{array}{c}\text { \# of } \\
\text { Epochs }\end{array}$ & $\begin{array}{l}\text { Best } \\
\text { Epoch }\end{array}$ & Band & $\begin{array}{c}\text { Minor } \\
\text { Axis (") }\end{array}$ & $\begin{array}{l}\text { Axis } \\
\text { Ratio }\end{array}$ & $\begin{array}{c}\text { Orientation } \\
\text { Angle }\left({ }^{\circ}\right)\end{array}$ & Multiple? $^{\mathrm{a}}$ \\
\hline J0846-7246 & 1 & $14-04-17$ & $\mathrm{H}$ & $0.041 \pm 0.001$ & $0.79 \pm 0.02$ & $158 \pm 6$ & $\mathrm{Y}$ \\
\hline J0847-7854 & 1 & $14-04-17$ & $\mathrm{H}$ & $0.053 \pm 0.004$ & $0.82 \pm 0.04$ & $26 \pm 1$ & $\mathrm{~N}$ \\
\hline J0903-6348 & 4 & $14-04-21$ & $\mathrm{H}$ & $0.038 \pm 0.001$ & $0.97 \pm 0.02$ & $84 \pm 47$ & $\mathrm{Y}$ \\
\hline J0911-5014 & 1 & $15-05-12$ & $\mathrm{H}$ & $0.033 \pm 0.001$ & $0.85 \pm 0.01$ & $101 \pm 3$ & $\mathrm{~N}$ \\
\hline J0931-5314 & 2 & $15-05-12$ & $\mathrm{H}$ & $0.034 \pm 0.002$ & $0.87 \pm 0.04$ & $103 \pm 7$ & $\mathrm{Y}$ \\
\hline J0933-4848 & 1 & $14-04-17$ & $\mathrm{H}$ & $0.041 \pm 0.004$ & $0.87 \pm 0.06$ & $11 \pm 14$ & $\mathrm{~N}$ \\
\hline J1025-4918 & 2 & $15-05-10$ & $\mathrm{H}$ & $0.032 \pm 0.001$ & $0.71 \pm 0.00$ & $37 \pm 3$ & $\mathrm{~N}$ \\
\hline J1123-5257 & 1 & $15-05-11$ & $\mathrm{H}$ & $0.038 \pm 0.001$ & $0.80 \pm 0.03$ & $50 \pm 6$ & $\mathrm{~N}$ \\
\hline $\mathrm{J} 1145-5520$ & 2 & $15-05-10$ & $\mathrm{H}$ & $0.033 \pm 0.002$ & $0.84 \pm 0.04$ & $164 \pm 5$ & $\mathrm{~N}$ \\
\hline J1149-7851 & 2 & $14-04-21$ & $\mathrm{H}$ & $0.034 \pm 0.001$ & $0.82 \pm 0.02$ & $173 \pm 4$ & $\mathrm{~N}$ \\
\hline J1223-4606 & 1 & $14-04-17$ & $\mathrm{H}$ & $0.043 \pm 0.002$ & $0.88 \pm 0.02$ & $47 \pm 7$ & $\mathrm{~N}$ \\
\hline J1231-4558 & 2 & $15-05-12$ & $\mathrm{H}$ & $0.036 \pm 0.003$ & $0.84 \pm 0.02$ & $95 \pm 6$ & $\mathrm{Y}$ \\
\hline $\mathrm{J} 1234-4538$ & 1 & $15-05-12$ & $\mathrm{H}$ & $0.034 \pm 0.001$ & $0.75 \pm 0.03$ & $114 \pm 5$ & $\mathrm{Y}$ \\
\hline J1235-4136 & 1 & $15-05-11$ & $\mathrm{H}$ & $0.042 \pm 0.004$ & $0.90 \pm 0.10$ & $4 \pm 41$ & $\mathrm{~N}$ \\
\hline J1354-7121 & 1 & $15-05-11$ & $\mathrm{H}$ & $0.033 \pm 0.001$ & $0.85 \pm 0.02$ & $51 \pm 10$ & $\mathrm{~N}$ \\
\hline $\mathrm{J} 1425-4113$ & 1 & $15-05-12$ & $\mathrm{H}$ & $0.047 \pm 0.005$ & $0.63 \pm 0.13$ & $47 \pm 6$ & $\mathrm{Y}$ \\
\hline $\mathrm{J} 1428-7430$ & 1 & $15-05-11$ & $\mathrm{H}$ & $0.030 \pm 0.000$ & $0.80 \pm 0.02$ & $30 \pm 5$ & $\mathrm{~N}$ \\
\hline J1516-5855 & 1 & $15-05-10$ & $\mathrm{H}$ & $0.033 \pm 0.001$ & $0.85 \pm 0.01$ & $96 \pm 7$ & $\mathrm{Y}$ \\
\hline J1524-4929 & 1 & $15-05-12$ & $\mathrm{H}$ & $0.034 \pm 0.001$ & $0.91 \pm 0.05$ & $71 \pm 6$ & $\mathrm{Y}$ \\
\hline J1657-5343 & 1 & $15-05-10$ & $\mathrm{H}$ & $0.034 \pm 0.000$ & $0.84 \pm 0.04$ & $94 \pm 4$ & $\mathrm{Y}$ \\
\hline J1708-6936 & 1 & $15-05-11$ & $\mathrm{H}$ & $0.038 \pm 0.000$ & $0.89 \pm 0.03$ & $167 \pm 8$ & $\mathrm{Y}$ \\
\hline J1729-5014 & 1 & $15-05-11$ & $\mathrm{H}$ & $0.040 \pm 0.001$ & $0.71 \pm 0.03$ & $12 \pm 1$ & $\mathrm{Y}$ \\
\hline J1814-3246 & 1 & $15-05-12$ & $\mathrm{H}$ & $0.032 \pm 0.003$ & $0.88 \pm 0.04$ & $79 \pm 29$ & $\mathrm{~N}$ \\
\hline J1842-5554 & 1 & $15-05-10$ & $\mathrm{H}$ & $0.034 \pm 0.002$ & $0.90 \pm 0.06$ & $72 \pm 12$ & $\mathrm{~N}$ \\
\hline J1846-6210 & 1 & $15-05-10$ & $\mathrm{H}$ & $0.036 \pm 0.003$ & $0.91 \pm 0.03$ & $24 \pm 44$ & $\mathrm{~N}$ \\
\hline J1922-6310 & 2 & $15-05-10$ & $\mathrm{H}$ & $0.035 \pm 0.001$ & $0.96 \pm 0.02$ & $14 \pm 32$ & $\mathrm{~N}$ \\
\hline J1923-4606 & 2 & $15-05-11$ & $\mathrm{H}$ & $0.040 \pm 0.006$ & $0.85 \pm 0.05$ & $76 \pm 28$ & $\mathrm{~N}$ \\
\hline $\mathrm{J} 2007-5147$ & 1 & $15-05-11$ & $\mathrm{H}$ & $0.033 \pm 0.002$ & $0.79 \pm 0.01$ & $117 \pm 7$ & $\mathrm{~N}$ \\
\hline $\mathrm{J} 2042-5425$ & 2 & $15-05-11$ & $\mathrm{H}$ & $0.062 \pm 0.003$ & $0.89 \pm 0.08$ & $4 \pm 30$ & $\mathrm{~N}$ \\
\hline J2108-4244 & 2 & 14-11-11 & $\mathrm{Ks}$ & $0.042 \pm 0.001$ & $0.91 \pm 0.01$ & $91 \pm 4$ & $\mathrm{Y}$ \\
\hline $\mathrm{J} 2110-5811$ & 3 & $15-05-11$ & Ks & $0.057 \pm 0.004$ & $0.91 \pm 0.03$ & $152 \pm 9$ & $\mathrm{~N}$ \\
\hline J2114-4213 & 2 & $15-05-11$ & $\mathrm{H}$ & $0.053 \pm 0.002$ & $0.87 \pm 0.06$ & $178 \pm 11$ & $\mathrm{~N}$ \\
\hline J2116-6005 & 2 & $15-05-11$ & $\mathrm{H}$ & $0.056 \pm 0.005$ & $0.82 \pm 0.03$ & $23 \pm 23$ & $\mathrm{~N}$ \\
\hline J2121-6655 & 2 & $15-05-11$ & $\mathrm{H}$ & $0.041 \pm 0.002$ & $0.89 \pm 0.07$ & $51 \pm 46$ & $\mathrm{Y}$ \\
\hline $\mathrm{J} 2135-4218$ & 2 & $15-05-11$ & $\mathrm{H}$ & $0.052 \pm 0.001$ & $0.75 \pm 0.03$ & $21 \pm 3$ & $\mathrm{~N}$ \\
\hline $\mathrm{J} 2149-6413$ & 2 & $15-05-11$ & $\mathrm{H}$ & $0.064 \pm 0.005$ & $0.89 \pm 0.03$ & $131 \pm 26$ & $\mathrm{Y}$ \\
\hline J2202-6440 & 1 & $15-05-11$ & $\mathrm{H}$ & $0.031 \pm 0.001$ & $0.87 \pm 0.02$ & $15 \pm 8$ & $\mathrm{~N}$ \\
\hline $\mathrm{J} 2244-5413$ & 2 & $14-12-01$ & $\mathrm{H}$ & $0.045 \pm 0.004$ & $0.86 \pm 0.05$ & $137 \pm 3$ & $\mathrm{Y}$ \\
\hline J2246-7353 & 1 & $15-05-11$ & $\mathrm{H}$ & $0.040 \pm 0.002$ & $0.78 \pm 0.12$ & $111 \pm 5$ & $\mathrm{~N}$ \\
\hline $\mathrm{J} 2247-6920$ & 4 & $15-05-11$ & $\mathrm{H}$ & $0.034 \pm 0.005$ & $0.77 \pm 0.16$ & $137 \pm 27$ & $\mathrm{Y}$ \\
\hline $\mathrm{J} 2347-6517$ & 1 & $14-11-30$ & $\mathrm{Ks}$ & $0.043 \pm 0.000$ & $0.94 \pm 0.05$ & $28 \pm 15$ & $\mathrm{~N}$ \\
\hline $\mathrm{J} 2353-7056$ & 1 & $14-11-30$ & $\mathrm{Ks}$ & $0.047 \pm 0.001$ & $0.92 \pm 0.02$ & $4 \pm 1$ & $\mathrm{~N}$ \\
\hline
\end{tabular}

Note.

" $\mathrm{N}$ denotes "Single" and Y denotes "Multiple" within 4."0 based on our observations and known VBs and SBs from the literature.

(This table is available in machine-readable form.)

observations whenever available, and from an adjacent night otherwise. For Ks images, we use the master Ks-flat field from 2014 April supplied by the Magellan MagAO team, available through the online Clio user manual.

MagAO twilight flats are known to be unreliable (see, e.g., Morzinski et al. 2015). Thus, we compared photometric and astrometric measurements derived from several images of binaries that had been flat-fielded to the unflattened ones. In terms of astrometry, the two sets of images produce virtually identical values with indistinguishable precision. For the flux ratio measurements, flattening leads to a slight reduction in the errors. Therefore, we opt to use flat-fielded images for our entire analysis.

\section{YMG Binaries}

\subsection{Clio Binary Detections}

We visually examined every Clio as well as VisAO image in SAOImage DS9 (Joye \& Mandel 2003) at various contrasts to identify visual doubles among our targets. To the Clio images we fit triple bivariate Gaussian point-spread functions (PSFs) to all suspected sources using mpfitfun.pro, an IDL routine that uses a Levenberg-Marquardt technique to efficiently explore the chi-squared space of an arbitrary multi-parameter function (Markwardt 2009). This is similar to the algorithm described by Bowler \& Hillenbrand (2015). The fits are initialized by parameter estimates from visual inspection of the 


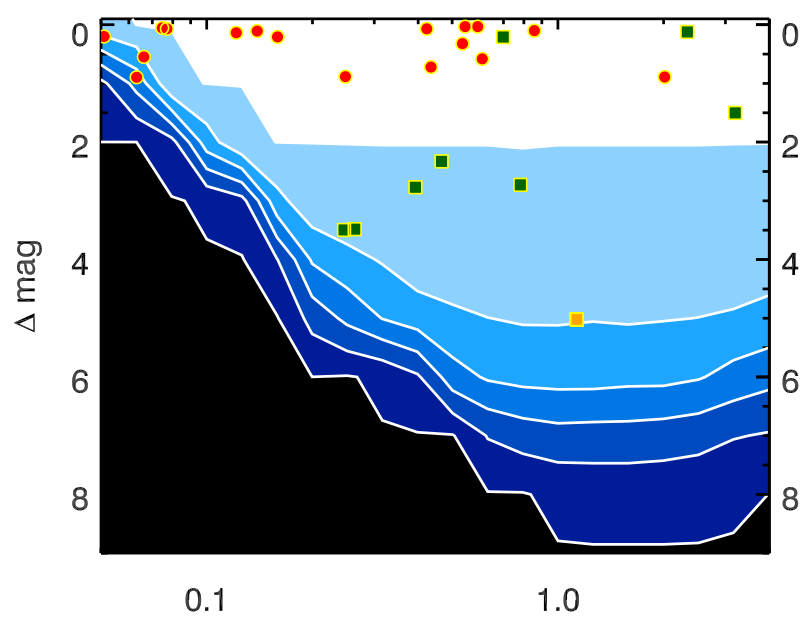

Angular Separation (")

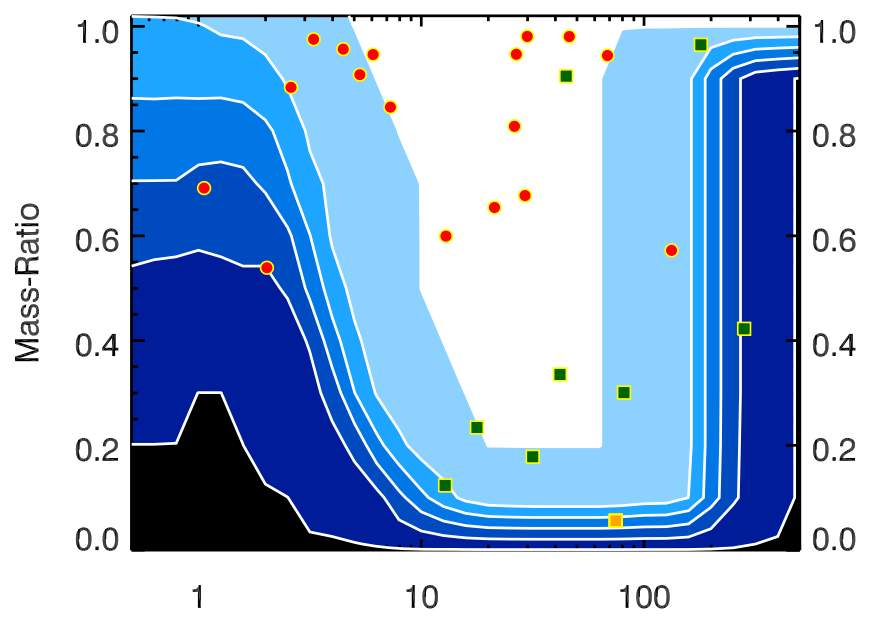

Projected Physical Separation (AU)

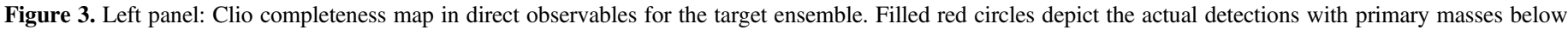

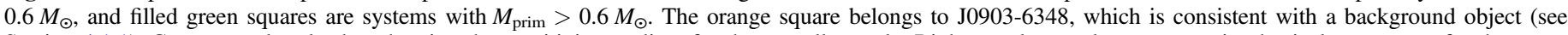

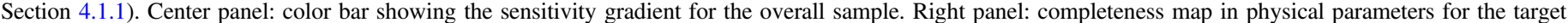
ensemble. Sections 5.1 and 5.2 describe the method for generating these maps.

images. For every target whose Clio or VisAO images in a particular epoch exhibit multiple clear visually identifiable components, we perform a multi-source fit to its Clio images. The PSFs of all components are constrained to share the same PSF shape. The centroid positions and the overall height scalings (which is proportional to the total flux) are allowed to vary independently for each component. Figure 2 shows an example fit to a tight binary.

For all images, we measure the minimum radius of the PSF cross-section at half-height. We compute the axis ratio of this cross-section as the minimum radius divided by the maximum radius. We also output the east of north orientation of the major axis. We give these parameters in Table 4 for all targets measured in their best epochs (determined by the depth of their contrast curves in the annulus between 0 !' 1 and 1 !" 0 ). For binaries we additionally measure their separation, position angle (PA), and flux ratio from the fitted Gaussians. Binary separation is the distance between the two fitted PSF centroids, in pixel units. This can be converted into angular units using the plate scale $(15.846 \pm 0.064 \mathrm{mas} / \mathrm{pixel}$, Morzinski et al. 2015), and then into physical units using the distance to the source (as reported in Table 3). When comparing the plate scale measured in 2012 (Morzinski et al. 2015) to that measured from data taken in 2015 November, the systematic uncertainty in the plate scale is approximately 0.1 mas/pixel. We use this value as the fiducial error in our plate scale. PA is the angle between the vector connecting the primary to the secondary centroid and north, measured counterclockwise (i.e., east of north). To obtain the true PA, the image angles are derotated using the derotation angle (ROTOFF) associated with that observation as well as the true north angle (NORTH_CLIO $=-1.80 \pm 0.34^{\circ}$; Morzinski et al. 2015) of the instrument. The flux ratio is determined as the overall secondary-to-primary PSF scaling factor, which is converted into $\Delta \mathrm{mag}$.

The final reported values are averaged over the independent measurements in the set of (four or eight) consecutive images taken for each target at each epoch. We note that in the case of PA, we take the average in complex space. We take the measurement errors to be the standard error of the mean for each epoch. The final errors combine, in quadrature, the measurement errors and uncertainties from the instrument calibrations. Physical quantities also include uncertainties due to the distances from the literature.

For all apparently single sources where the average PSF axis ratio at a given epoch is lower than 0.72 (corresponding to a fractional elongation of $40 \%$ ), we perform a two-source fit. Based on our wider binary detections, we define a secure close binary detection to be one in which the standard deviations in the measured binaries quantities meet the following criteria:

1. Position angle: $\sigma_{\mathrm{PA}}<15^{\circ}$,

2. Flux ratio: $\sigma_{F_{s} / F_{p}}<0.1$, and

3. Separation $\sigma_{\text {sep }} /$ Sep $<20 \%$.

In addition, we require that the one-source elongated PSF has an orientation angle that is consistent with the two-source PA within $15^{\circ}$, and that the two-source PSF is more round than the one-source counterpart (i.e., axis ratio increases toward 1). This procedure allowed us to obtain robust astrometric measurements for three very tight $(<70$ mas $)$ binaries that were also resolved in VisAO (see Figure 6). Furthermore, if a binary is observed in one or more epochs, we perform a twosource fit to that object in all epochs.

In summary, our Clio images revealed 27 close $\left(<4^{\prime \prime}\right)$ visual doubles that were confidently fit by two-source solutions in 105 targets, of which 14 are new discoveries. The left panel of Figure 3 shows the distribution of these doubles as a function of $\Delta$ mag and projected angular separation. Snapshots of these visually detected pairs are found in Figures 4-6. For targets imaged in previous works, our multiplicity designation is mostly in agreement, except in individual cases, which are listed in Section 4.3.

The right panel of Figure 3 shows the detected binaries in physical units. To estimate the primary masses, we use the 2MASS magnitudes for each target in conjunction with the latest stellar isochrones for low-mass PMS stars from Baraffe et al. (2015), which assumes solar metallicity. Table 1 lists the isochrone ages adopted for each YMG. For single stars, this conversion is straightforward. For the doubles, we must account for the fact that the 2MASS magnitudes include 


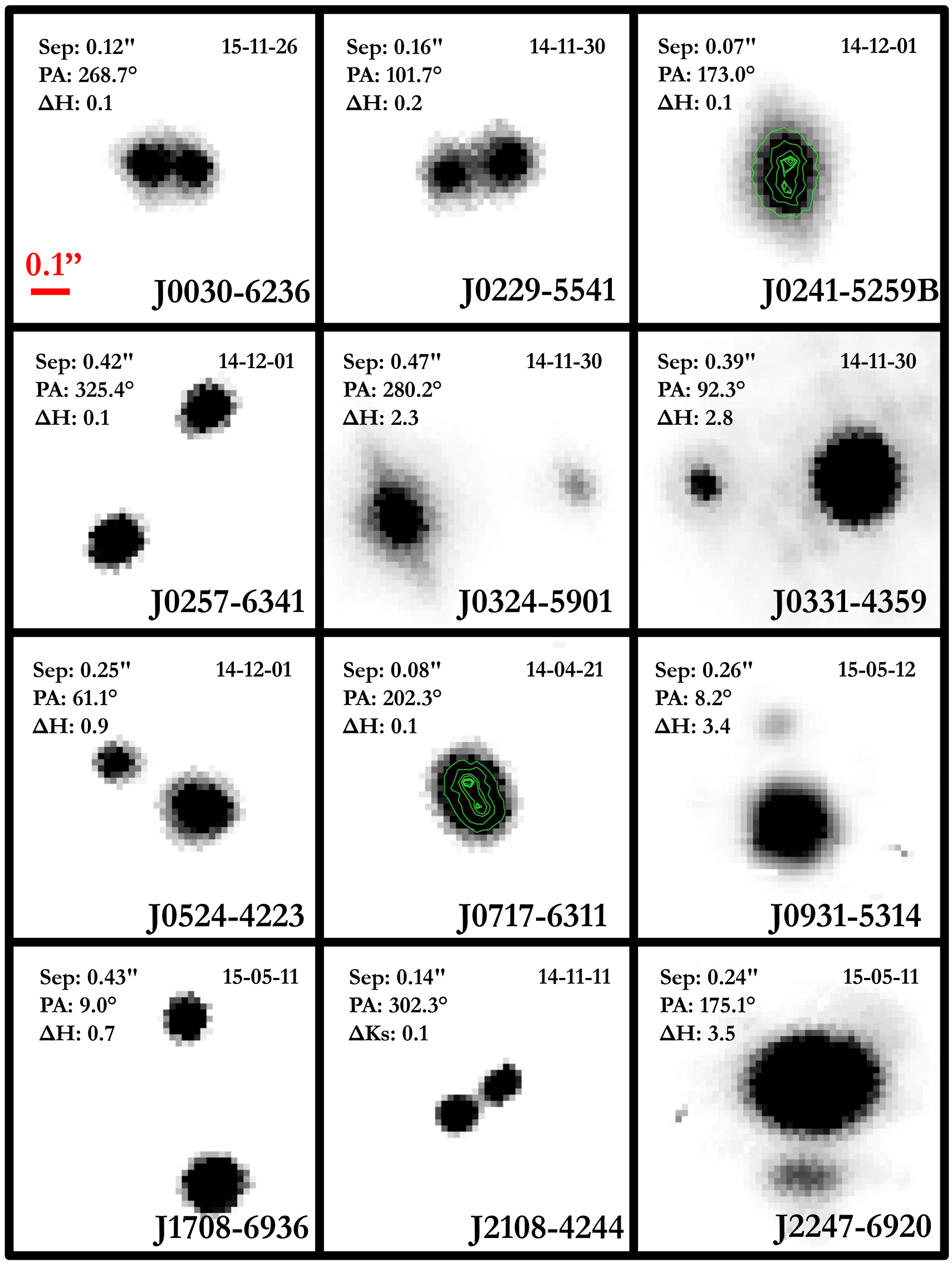

Figure 4. Clio images of binaries separated by less than 0 ! 5 . Each image is $0 . \prime 8 \times 0$." 8 . The images have been oriented so that north points up and east points left. The color scale is arbitrary for display purposes. Contour plots are shown whenever the binary nature is not obvious. 


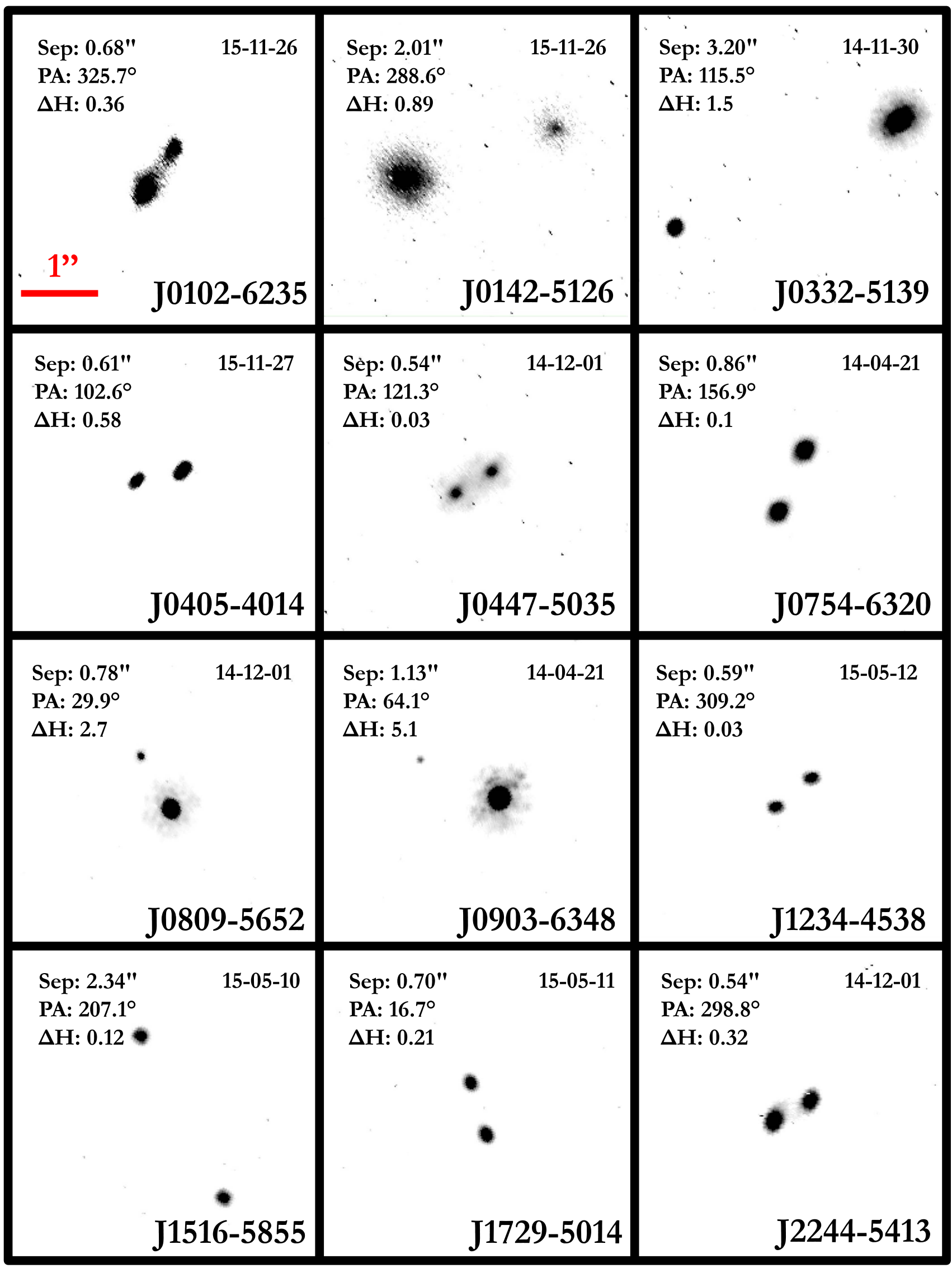

Figure 5. Clio images of binaries with angular separations between 0 ." 5 and 4 !. 0 . Each image is 4 .' $0 \times 4$.' 0 . The images have been oriented so that north points up and east points left. The color scale is arbitrary for display purposes. 
Table 5

Astrometric Measurements for VB Candidates from This Work

\begin{tabular}{|c|c|c|c|c|c|c|c|c|}
\hline Target Name & $\begin{array}{l}\mu_{\alpha} \cos \delta^{\mathrm{a}} \\
\left(\operatorname{mas}^{-1} \mathrm{yr}^{-1}\right)\end{array}$ & 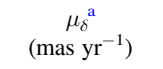 & Epoch & Band & Ang. Sep. $(")$ & $\operatorname{PA}\left({ }^{\circ}\right)$ & $\Delta \mathrm{mag}$ & Comove? ${ }^{\mathrm{d}}$ \\
\hline J0030-6236 & $95.2 \pm 0.9$ & $-48.0 \pm 0.9$ & $15-11-26$ & $\mathrm{H}$ & $0.121 \pm 0.001$ & $268.6 \pm 0.36$ & $0.14 \pm 0.04$ & $\mathrm{CO}$ \\
\hline J0102-6235 & $88.9 \pm 1.2$ & $-39.3 \pm 1.2$ & $\begin{array}{l}14-12-01 \\
15-11-26\end{array}$ & $\begin{array}{l}\mathrm{H} \\
\mathrm{H}\end{array}$ & $\begin{array}{l}0.566 \pm 0.044 \\
0.678 \pm 0.005\end{array}$ & $\begin{array}{l}332.7 \pm 2.05 \\
325.7 \pm 0.35\end{array}$ & $\begin{array}{l}0.27 \pm 0.08 \\
0.36 \pm 0.04\end{array}$ & B \\
\hline J0142-5126 & $66.8 \pm 4.2$ & $-12.7 \pm 4.2$ & $15-11-26$ & $\mathrm{H}$ & $2.014 \pm 0.014$ & $288.6 \pm 0.37$ & $0.89 \pm 0.23$ & $\ldots$ \\
\hline J0229-5541 & $91.3 \pm 3.4$ & $-16.0 \pm 3.4$ & $14-11-30$ & $\mathrm{H}$ & $0.159 \pm 0.001$ & $101.4 \pm 0.35$ & $0.21 \pm 0.04$ & $\ldots$ \\
\hline J0241-5259B & $91.6 \pm 1.1$ & $-3.6 \pm 1.0$ & $14-12-01$ & $\mathrm{H}$ & $0.075 \pm 0.001$ & $174.5 \pm 0.36$ & $0.05 \pm 0.01$ & $\ldots$ \\
\hline J0257-6341 & $64.1 \pm 1.9$ & $11.6 \pm 4.1$ & $14-12-01$ & $\mathrm{H}$ & $0.423 \pm 0.003$ & $325.4 \pm 0.35$ & $0.07 \pm 0.01$ & $\ldots$ \\
\hline J0324-5901 & $37.5 \pm 1.1$ & $9.6 \pm 1.1$ & $14-11-30$ & $\mathrm{H}$ & $0.466 \pm 0.003$ & $280.2 \pm 0.34$ & $2.33 \pm 0.01$ & $\mathrm{CO}$ \\
\hline J0331-4359 & $85.0 \pm 1.4$ & $-8.2 \pm 1.9$ & $14-11-30$ & $\mathrm{H}$ & $0.393 \pm 0.003$ & $92.3 \pm 0.34$ & $2.77 \pm 0.01$ & $\mathrm{CO}$ \\
\hline J0332-5139 & $38.2 \pm 1.3$ & $11.6 \pm 1.3$ & $14-11-30$ & $\mathrm{H}$ & $3.199 \pm 0.024$ & $115.5 \pm 0.34$ & $1.50 \pm 0.03$ & I \\
\hline J0405-4014 & $71.6 \pm 2.0$ & $-0.8 \pm 2.1$ & $15-11-27$ & $\mathrm{H}$ & $0.609 \pm 0.004$ & $102.6 \pm 0.34$ & $0.58 \pm 0.04$ & $\ldots$ \\
\hline J0447-5035 & $46.7 \pm 2.6$ & $19.8 \pm 2.6$ & $14-12-01$ & $\mathrm{H}$ & $0.544 \pm 0.004$ & $121.3 \pm 0.34$ & $0.03 \pm 0.03$ & $\mathrm{CO}$ \\
\hline J0524-4223 & $3.2 \pm 1.8$ & $-13.5 \pm 1.6$ & $14-12-01$ & $\mathrm{H}$ & $0.248 \pm 0.002$ & $61.0 \pm 0.37$ & $0.88 \pm 0.01$ & $\mathrm{CO}$ \\
\hline J0533-4257 & $-18.6 \pm 3.1$ & $43.1 \pm 3.5$ & $\begin{array}{l}14-12-01 \\
15-11-26\end{array}$ & $\begin{array}{l}\mathrm{H} \\
\mathrm{H}\end{array}$ & $\begin{array}{c}0.066 \pm 0.000 \\
\ldots\end{array}$ & $\begin{array}{c}222.2 \pm 1.09 \\
\ldots\end{array}$ & $\begin{array}{c}0.55 \pm 0.02 \\
\ldots\end{array}$ & $\ldots$ \\
\hline J0717-6311 & $-12.0 \pm 1.4$ & $45.8 \pm 1.4$ & $\begin{array}{l}14-04-21 \\
15-11-26\end{array}$ & $\begin{array}{l}\mathrm{H} \\
\mathrm{H}\end{array}$ & $\begin{array}{c}0.077 \pm 0.001 \\
\ldots{ }^{c}\end{array}$ & $\begin{array}{c}203.2 \pm 0.41 \\
\ldots\end{array}$ & $\begin{array}{c}0.07 \pm 0.01 \\
\ldots\end{array}$ & $\ldots$ \\
\hline J0754-6320 & $-9.4 \pm 2.5$ & $28.7 \pm 2.5$ & $\begin{array}{l}14-04-21 \\
15-11-26\end{array}$ & $\begin{array}{l}\mathrm{H} \\
\mathrm{H}\end{array}$ & $\begin{array}{l}0.857 \pm 0.005 \\
0.859 \pm 0.005\end{array}$ & $\begin{array}{l}156.9 \pm 0.34 \\
156.8 \pm 0.34\end{array}$ & $\begin{array}{l}0.10 \pm 0.01 \\
0.11 \pm 0.03\end{array}$ & $\mathrm{C}$ \\
\hline J0809-5652 & $-8.1 \pm 1.6$ & $19.6 \pm 1.1$ & $\begin{array}{l}14-12-01 \\
15-11-26\end{array}$ & $\begin{array}{l}\mathrm{H} \\
\mathrm{H}\end{array}$ & $\begin{array}{l}0.782 \pm 0.005 \\
0.785 \pm 0.005\end{array}$ & $\begin{array}{l}29.9 \pm 0.34 \\
29.7 \pm 0.34\end{array}$ & $\begin{array}{l}2.73 \pm 0.01 \\
2.71 \pm 0.02\end{array}$ & $\mathrm{C}$ \\
\hline J0903-6348 & $-33.3 \pm 1.4$ & $33.6 \pm 1.4$ & $\begin{array}{l}14-04-21 \\
14-11-30 \\
15-05-10 \\
15-11-26 \\
\end{array}$ & $\begin{array}{l}\mathrm{H} \\
\mathrm{H} \\
\mathrm{H} \\
\mathrm{H}\end{array}$ & $\begin{array}{l}1.132 \pm 0.007 \\
1.124 \pm 0.008 \\
1.149 \pm 0.010 \\
1.129 \pm 0.007\end{array}$ & $\begin{array}{l}64.1 \pm 0.34 \\
64.4 \pm 0.34 \\
66.0 \pm 0.37 \\
65.8 \pm 0.35\end{array}$ & $\begin{array}{l}5.02 \pm 0.03 \\
5.04 \pm 0.07 \\
5.01 \pm 0.04 \\
5.18 \pm 0.02\end{array}$ & B \\
\hline J0931-5314 & $-20.8 \pm 1.5$ & $3.4 \pm 3.4$ & $\begin{array}{r}15-05-12 \\
15-11-26 \\
\end{array}$ & $\begin{array}{l}\mathrm{H} \\
\mathrm{H} \\
\end{array}$ & $\begin{array}{l}0.264 \pm 0.002 \\
0.236 \pm 0.011 \\
\end{array}$ & $\begin{array}{r}8.2 \pm 0.34 \\
13.0 \pm 2.39 \\
\end{array}$ & $\begin{array}{l}3.48 \pm 0.10 \\
3.36 \pm 0.28 \\
\end{array}$ & $\mathrm{CO}$ \\
\hline J1234-4538 & $-47.5 \pm 1.3$ & $-20.2 \pm 0.8$ & $15-05-12$ & $\mathrm{H}$ & $0.591 \pm 0.004$ & $309.2 \pm 0.34$ & $0.03 \pm 0.03$ & $\mathrm{CO}$ \\
\hline J1516-5855 & $-42.8 \pm 3.4^{\mathrm{b}}$ & $-44.2 \pm 3.2^{b}$ & $15-05-10$ & $\mathrm{H}$ & $2.337 \pm 0.015$ & $207.1 \pm 0.34$ & $0.12 \pm 0.06$ & $\mathrm{C}$ \\
\hline $\mathrm{J} 1657-5343$ & $-13.0 \pm 6.3$ & $-85.1 \pm 2.2$ & $15-05-10$ & $\mathrm{H}$ & $0.051 \pm 0.000$ & $219.7 \pm 0.47$ & $0.20 \pm 0.01$ & $\ldots$ \\
\hline J1708-6936 & $-54.6 \pm 1.7$ & $-81.1 \pm 1.7$ & $15-05-11$ & $\mathrm{H}$ & $0.435 \pm 0.003$ & $9.1 \pm 0.34$ & $0.72 \pm 0.02$ & $\mathrm{C}$ \\
\hline J1729-5014 & $-5.8 \pm 1.5$ & $-62.7 \pm 5.1$ & $15-05-11$ & $\mathrm{H}$ & $0.699 \pm 0.005$ & $16.7 \pm 0.34$ & $0.21 \pm 0.05$ & $\mathrm{CO}$ \\
\hline J2108-4244 & $33.3 \pm 1.6$ & $-99.6 \pm 1.5$ & $\begin{array}{r}14-11-11 \\
15-11-27 \\
\end{array}$ & $\begin{array}{l}\mathrm{Ks} \\
\mathrm{H}\end{array}$ & $\begin{array}{l}0.139 \pm 0.001 \\
0.129 \pm 0.001\end{array}$ & $\begin{array}{l}302.3 \pm 0.35 \\
287.1 \pm 0.35\end{array}$ & $\begin{array}{l}0.11 \pm 0.04 \\
0.13 \pm 0.04\end{array}$ & $\mathrm{CO}$ \\
\hline J2121-6655 & $97.2 \pm 1.1$ & $-104.1 \pm 1.6$ & $\begin{array}{l}15-05-11 \\
15-11-26\end{array}$ & $\begin{array}{l}\mathrm{H} \\
\mathrm{H}\end{array}$ & $\begin{array}{c}0.063 \pm 0.001 \\
\ldots\end{array}$ & $\begin{array}{c}301.6 \pm 4.48 \\
\ldots\end{array}$ & $\begin{array}{c}0.90 \pm 0.15 \\
\ldots\end{array}$ & $\ldots$ \\
\hline $\mathrm{J} 2244-5413$ & $70.7 \pm 1.3$ & $-60.0 \pm 1.3$ & $\begin{array}{l}14-12-01 \\
15-11-26\end{array}$ & $\begin{array}{l}\mathrm{H} \\
\mathrm{H}\end{array}$ & $\begin{array}{l}0.535 \pm 0.004 \\
0.520 \pm 0.003\end{array}$ & $\begin{array}{l}298.8 \pm 0.35 \\
298.1 \pm 0.34\end{array}$ & $\begin{array}{l}0.32 \pm 0.01 \\
0.28 \pm 0.02\end{array}$ & $\mathrm{CO}$ \\
\hline J2247-6920 & $70.9 \pm 1.6$ & $-58.9 \pm 1.8$ & $\begin{array}{l}14-12-01 \\
15-05-11 \\
15-11-26\end{array}$ & $\begin{array}{l}\mathrm{H} \\
\mathrm{H} \\
\mathrm{H}\end{array}$ & $\begin{array}{l}0.146 \pm 0.015^{\mathrm{e}} \\
0.246 \pm 0.002 \\
0.228 \pm 0.003\end{array}$ & $\begin{array}{l}161.2 \pm 4.45 \\
175.2 \pm 0.35 \\
166.0 \pm 0.59\end{array}$ & $\begin{array}{l}3.12 \pm 0.09 \\
3.50 \pm 0.11 \\
3.42 \pm 0.19\end{array}$ & $\mathrm{CO}$ \\
\hline
\end{tabular}

Notes.

a Proper motions from the UCAC4 catalog (Zacharias et al. 2012) unless otherwise specified.

b Proper motion from the NOMAD catalog (Zacharias et al. 2005), which was used in the analysis by Malo et al. (2013).

${ }^{\mathrm{c}}$ No data are reported for epochs in which our fit could not reliably recover both components.

${ }^{\mathrm{d}} \mathrm{C}$ : comoving; CO: comoving with orbital motion; B: background; I: indeterminate.

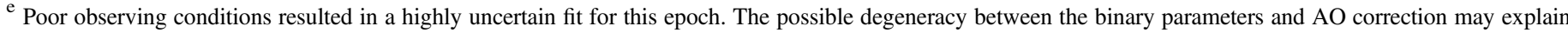
why the measurements are inconsistent with the other epochs.

(This table is available in machine-readable form.) 
Table 6

System Properties of VisAO Visual Binaries

\begin{tabular}{lcccccccc}
\hline \hline $\begin{array}{l}\text { Target } \\
\text { Name }\end{array}$ & SpT & $\begin{array}{c}\text { Dist. } \\
(\mathrm{pc})\end{array}$ & Epoch & $\begin{array}{c}\text { Equal } \\
\text { Mass? }\end{array}$ & $\begin{array}{c}M_{\star}{ }^{\text {a }} \\
\left(M_{\odot}\right)\end{array}$ & $\begin{array}{c}M_{p}{ }^{\text {b }} \\
\left(M_{\odot}\right)\end{array}$ & $\begin{array}{c}\text { Ang. Sep. } \\
(\mathrm{mas})\end{array}$ & $\begin{array}{c}\text { Proj. Phys. } \\
\text { Sep. }(\mathrm{au})\end{array}$ \\
\hline J0017-7032 & M0.5 & $63 \pm 4$ & $14-11-10$ & no & 0.8 & 0.8 & 58 \\
J0241-5259A & K6.0 & $43 \pm 1$ & $14-12-01$ & yes & 0.9 & 0.7 & 40 \\
\hline
\end{tabular}

Notes.

${ }^{a}$ Mass derived from single-passband photometry, age, distance, and pre-MS isochrones (Baraffe et al. 2015), assuming the star is single.

b Same as "a", but now taking into account whether the star appears to be an equal-mass binary.

${ }^{c}$ See Section 5.2 for explanation.

both unresolved visual components. We split the corresponding 2MASS flux according to the flux ratios we measure in the Clio $\mathrm{H}$ or $\mathrm{K}$ band for the best epoch and use the evolutionary models from Baraffe et al. (2015) for both components. Cases in which a tertiary and/or a known SB exists are described individually in Section 4.3.

\subsubsection{Validation}

There are two ways in which we could validate the Clio visual binaries as bound companions. First, in combination with published information from the literature (Chauvin et al. 2010; J12; Elliott et al. 2015; J17), we have multi-epoch astrometric measurements for 18 visual doubles that can be used to confirm whether they are comoving and therefore bound. Table 5 presents the astrometric measurements from this work. The figures in the Appendix show the measured positions of the binaries as compared to the expectation for a background star. This test shows that 15 of the 18 targets are comoving. For J0332-5139 this test is inconclusive, and we reject the remaining two visual companions, J0102-6235 and J0903-6348, as likely background objects (also see Section 4.3).

Second, given the spatial distribution of stars in the galaxy, we can compute the chance alignment probability of a given pair of sources with the measured angular separation and magnitudes. We use the TRILEGAL stellar population synthesis code to calculate the density of stars in the $0.5 \mathrm{deg}^{2}$ field around each VB candidate. We compute $P_{\text {bound }}$ for each binary following Duchêne et al. (2001; see Section 3.1.2 therein). We find that all binaries have essentially $100 \%$ bound probability. This is due to their small angular separations, the relatively high flux ratios, and the distributions in the sky, which are generally out of the galactic plane. A summary of the chance alignment probabilities (that is, $\left.P_{\text {unbound }}=100 \%-P_{\text {bound }}\right)$ is recorded in Table 8 .

\subsection{Other Binaries}

Two additional new, close, visual companions were apparent through the VisAO camera, but were not resolved in their Clio images. Table 6 gives their approximate properties, and their VisAO images are shown in Figure 7. Since only $4 \%$ of the light was sent to the VisAO camera, accurate photometric measurements on these discoveries have not been attempted. Notably, both objects have been flagged as probable binaries by the BANYAN YMG membership analysis from Malo et al. (2013).

Several of the targets are also previously known spectroscopic binaries. These are listed in Table 7.
Table 7

List of Spectroscopic Binaries in Our Sample

\begin{tabular}{lccclr}
\hline \hline $\begin{array}{l}\text { Target } \\
\text { Name }\end{array}$ & SpT & $\begin{array}{c}M_{\text {prim }} \\
\left(M_{\odot}\right)\end{array}$ & $\begin{array}{l}M_{\text {sec }} \\
\left(M_{\odot}\right)\end{array}$ & $\begin{array}{l}\text { SB } \\
\text { Type }\end{array}$ & References \\
\hline J0222-6022 & M4.0 & 0.47 & $\ldots$ & SB1 & 5 \\
J0451-4647 & M0.0 & 0.59 & 0.48 & SB2 & 1 \\
J0533-5117 & K7.0 & 0.70 & 0.53 & SB2 & 1 \\
J0818-7239 & M0.0 & 0.42 & 0.42 & SB2 & 2 \\
J0846-7246 & K7.0 & 0.61 & 0.61 & SB2 & 2 \\
J1231-4558 & M3.0 & 0.39 & 0.39 & SB2 & 3,4 \\
J1425-4113 & M2.5 & 0.73 & 0.73 & SB2 & 2 \\
J1524-4929 & M2.0 & 0.54 & $\ldots$ & SB1 & 2 \\
J2247-6920 & K6.0 & 0.68 & $\ldots$ & SB1 & 2 \\
\hline
\end{tabular}

Note. For a detailed explanation of the calculation of $M_{\text {prim }}$ and $M_{\text {sec }}$, see Sections 4.3 and 5.2.

References. 1. Kraus et al. (2014), 2. Malo et al. (2014), 3. Jayawardhana et al. (2006), 4. Elliott et al. (2014), 5. this work: we infer this target is an SB1 based on the fact that following independent RV measurements, Kraus et al. (2014) and Malo et al. (2014) come to different conclusions regarding this object's membership in TucHor.

\subsection{Individual Targets of Note}

J0030-6236 (see Figure 8). This object consists of a tight binary (separation $\sim 0$." $12 \sim 5.3 \mathrm{au}, \Delta H \sim 0.14$ ) and a fainter wide visual tertiary (separation $\sim 4$ ". $45 \sim 200 \mathrm{au}, \Delta H \sim 2.0$ relative to the inner binary). The inferred masses of the components are $0.54,0.49$, and $0.23 M_{\odot}$. Note that the tertiary is outside the $4^{\prime \prime}$ limit we set for our statistical analysis in Section 5, but by virtue of its inner binary, this system is counted as a multiple.

J0102-6235. Kraus et al. (2014) describe this object as a member of TucHor and an SB3. We observe this target to be a 0 " 5 visual double. The comoving analysis (see Table 5 and Figure 14 in Appendix) suggests that one of the components is a background object. Since the properties of the combined star indicate that it is a YMG member, we infer that the brighter component is the member and the fainter component is the background object. One hypothesis is that the background object is an $\mathrm{M}$ giant that corresponds to one of the observed SB3 components. Future spectroscopic observations could resolve this issue. In the meantime, we exclude this target from our statistical analysis.

J0222-6022. Kraus et al. (2014) find that their single-epoch $\mathrm{RV}$ measurement is not consistent with this source being a member of TucHor, unless it is an SB1. In contrast, Malo et al. (2014) deem it a very secure TucHor member based on an independent RV measurement. From these discrepant classifications, we posit that this system is in fact a single-lined 
Table 8

System Properties of Visual Binaries Observed by Clio

\begin{tabular}{|c|c|c|c|c|c|c|c|c|c|c|c|c|c|c|c|}
\hline $\begin{array}{l}\text { Target } \\
\text { Name }\end{array}$ & $\mathrm{SpT}$ & $\begin{array}{l}\text { Dist. } \\
\text { (pc) }\end{array}$ & Epoch & Band & $\begin{array}{l}\text { Ang. Sep. } \\
\left({ }^{\prime \prime}\right)\end{array}$ & $\begin{array}{l}\mathrm{PA}^{\mathrm{a}} \\
\left({ }^{\circ}\right)\end{array}$ & $\Delta \mathrm{mag}$ & $\begin{array}{l}\text { Co- } \\
\text { move? }\end{array}$ & $\begin{array}{l}P_{\text {unbound }} \\
(\%)\end{array}$ & $\begin{array}{l}\text { Proj. Phys. } \\
\text { Sep. (au) }\end{array}$ & $\begin{array}{c}M_{\text {prim }}{ }^{\mathrm{b}} \\
\left(M_{\odot}\right)\end{array}$ & $\begin{array}{l}M_{\mathrm{sec}}^{\mathrm{b}} \\
\left(M_{\odot}\right)\end{array}$ & $q$ & $\begin{array}{l}\text { Known } \\
\text { Binary? }\end{array}$ & Notes \\
\hline \multicolumn{16}{|c|}{ TucHor } \\
\hline J0030-6236 & M2.2 & $44 \pm 2$ & $15-11-26$ & $\mathrm{H}$ & $0.121 \pm 0.001$ & $268.6 \pm 0.36$ & $0.14 \pm 0.04$ & $\mathrm{CO}$ & 0.0000 & $5.3 \pm 0.2$ & 0.54 & 0.49 & 0.9 & yes & 1,2 \\
\hline J0102-6235 & M2.9 & $46 \pm 2$ & $15-11-26$ & $\mathrm{H}$ & $0.678 \pm 0.005$ & $325.7 \pm 0.35$ & $0.36 \pm 0.04$ & B & 0.0000 & $31.0 \pm 1.4$ & $\ldots$ & $\ldots$ & $\ldots$ & yes & 2 \\
\hline J0229-5541 & M4.8 & $46 \pm 2$ & 14-11-30 & $\mathrm{H}$ & $0.159 \pm 0.001$ & $101.4 \pm 0.35$ & $0.21 \pm 0.04$ & & 0.0000 & $7.3 \pm 0.3$ & 0.11 & 0.09 & 0.8 & no & \\
\hline J0241-5259B & M2.5 & $44 \pm 3$ & 14-12-01 & $\mathrm{H}$ & $0.075 \pm 0.001$ & $174.5 \pm 0.36$ & $0.05 \pm 0.01$ & $\ldots$ & 0.0000 & $3.3 \pm 0.2$ & 0.56 & 0.54 & 1.0 & no & 3 \\
\hline J0257-6341 & M3.6 & $63 \pm 3$ & 14-12-01 & $\mathrm{H}$ & $0.423 \pm 0.003$ & $325.4 \pm 0.35$ & $0.07 \pm 0.01$ & & 0.0000 & $26.7 \pm 1.3$ & 0.32 & 0.30 & 0.9 & no & \\
\hline J0331-4359 & K6.0 & $45 \pm 1$ & 14-11-30 & $\mathrm{H}$ & $0.393 \pm 0.003$ & $92.3 \pm 0.34$ & $2.77 \pm 0.01$ & $\mathrm{CO}$ & 0.0001 & $17.8 \pm 0.4$ & 0.73 & 0.17 & 0.2 & yes & $2,4,5$ \\
\hline J0405-4014 & M4.2 & $48 \pm 3$ & $15-11-27$ & $\mathrm{H}$ & $0.609 \pm 0.004$ & $102.6 \pm 0.34$ & $0.58 \pm 0.04$ & & 0.0001 & $29.2 \pm 0.6$ & 0.30 & 0.20 & 0.7 & no & $\ldots$ \\
\hline J0447-5035 & M4.0 & $55 \pm 3$ & 14-12-01 & $\mathrm{H}$ & $0.544 \pm 0.004$ & $121.3 \pm 0.34$ & $0.03 \pm 0.03$ & $\mathrm{CO}$ & 0.0000 & $29.9 \pm 1.6$ & 0.28 & 0.28 & 1.0 & yes & 2 \\
\hline J1708-6936 & M3.5 & $49 \pm 3$ & 15-05-11 & $\mathrm{H}$ & $0.435 \pm 0.003$ & $9.1 \pm 0.34$ & $0.72 \pm 0.02$ & $\mathrm{C}$ & 0.0001 & $21.3 \pm 1.3$ & 0.54 & 0.35 & 0.7 & yes & 2 \\
\hline J2108-4244 & M4.4 & $44 \pm 2$ & 14-11-11 & Ks & $0.139 \pm 0.001$ & $302.3 \pm 0.35$ & $0.11 \pm 0.04$ & $\mathrm{CO}$ & 0.0000 & $6.1 \pm 0.3$ & 0.20 & 0.19 & 0.9 & no & \\
\hline J2244-5413 & M4.0 & $49 \pm 4$ & 14-12-01 & $\mathrm{H}$ & $0.535 \pm 0.004$ & $298.8 \pm 0.35$ & $0.32 \pm 0.01$ & $\mathrm{CO}$ & 0.0000 & $26.2 \pm 2.1$ & 0.42 & 0.34 & 0.8 & yes & 2,6 \\
\hline J2247-6920 & K6.0 & $52 \pm 1$ & 15-05-11 & $\mathrm{H}$ & $0.246 \pm 0.002$ & $175.2 \pm 0.35$ & $3.50 \pm 0.11$ & $\mathrm{CO}$ & 0.0001 & $12.8 \pm 0.3$ & 0.68 & 0.08 & 0.1 & no & 7,8 \\
\hline \multicolumn{16}{|c|}{$\mathrm{Col}$} \\
\hline J0142-5126 & M6.5 & $66 \pm 6$ & $15-11-26$ & $\mathrm{H}$ & $2.014 \pm 0.014$ & $288.6 \pm 0.37$ & $0.89 \pm 0.23$ & & 0.0022 & $132.9 \pm 2.2$ & 0.21 & 0.12 & 0.6 & no & $\ldots$ \\
\hline J0324-5901 & K7.0 & $90 \pm 5$ & $14-11-30$ & $\mathrm{H}$ & $0.466 \pm 0.003$ & $280.2 \pm 0.34$ & $2.33 \pm 0.01$ & $\mathrm{CO}$ & 0.0002 & $42.0 \pm 2.3$ & 0.78 & 0.26 & 0.3 & yes & 2,4 \\
\hline J0332-5139 & M2.0 & $88 \pm 5$ & $14-11-30$ & $\mathrm{H}$ & $3.198 \pm 0.024$ & $115.5 \pm 0.34$ & $1.50 \pm 0.03$ & I & 0.0064 & $281.4 \pm 16.1$ & 0.62 & 0.26 & 0.4 & yes & 2,4 \\
\hline J0717-6311 & M2.0 & $58 \pm 4$ & 14-04-21 & $\mathrm{H}$ & $0.077 \pm 0.001$ & $203.2 \pm 0.41$ & $0.07 \pm 0.01$ & $\ldots$ & 0.0000 & $4.5 \pm 0.3$ & 0.39 & 0.37 & 1.0 & no & 4 \\
\hline \multicolumn{16}{|c|}{ bPic } \\
\hline J0533-4257 & M4.5 & $16 \pm 4$ & 14-12-01 & $\mathrm{H}$ & $0.066 \pm 0.000$ & $222.2 \pm 1.09$ & $0.55 \pm 0.02$ & $\ldots$ & 0.0000 & $1.1 \pm 0.3$ & 0.17 & 0.12 & 0.7 & no & 3 \\
\hline J1657-5343 & M3.0 & $51 \pm 3$ & $15-05-10$ & $\mathrm{H}$ & $0.051 \pm 0.000$ & $219.7 \pm 0.47$ & $0.20 \pm 0.01$ & & 0.0000 & $2.6 \pm 0.2$ & 0.53 & 0.47 & 0.9 & no & 4 \\
\hline J1729-5014 & M3.0 & $64 \pm 5$ & $15-05-11$ & $\mathrm{H}$ & $0.699 \pm 0.005$ & $16.7 \pm 0.34$ & $0.21 \pm 0.05$ & $\mathrm{CO}$ & 0.0003 & $44.7 \pm 3.5$ & 0.64 & 0.58 & 0.9 & yes & $3,5,9,13$ \\
\hline J2121-6655 & $\mathrm{K} 7.0$ & $32 \pm 1$ & 15-05-11 & $\mathrm{H}$ & $0.063 \pm 0.001$ & $301.6 \pm 4.48$ & $0.90 \pm 0.15$ & $\ldots$ & 0.0000 & $2.0 \pm 0.1$ & 0.55 & 0.30 & 0.5 & no & 4 \\
\hline \multicolumn{16}{|c|}{$\operatorname{Arg}$} \\
\hline J1516-5855 & K7.0 & $77 \pm 3$ & $15-05-10$ & $\mathrm{H}$ & $2.337 \pm 0.015$ & $207.1 \pm 0.34$ & $0.12 \pm 0.06$ & $\mathrm{C}$ & 0.0116 & $179.9 \pm 7.1$ & 0.67 & 0.65 & 1.0 & yes & 4,13 \\
\hline \multicolumn{16}{|c|}{$\mathrm{Car}$} \\
\hline J0754-6320 & M3.0 & $80 \pm 5$ & 14-04-21 & $\mathrm{H}$ & $0.857 \pm 0.005$ & $156.9 \pm 0.34$ & $0.10 \pm 0.01$ & $\mathrm{C}$ & 0.0001 & $68.6 \pm 4.3$ & 0.45 & 0.43 & 0.9 & yes & 2,3 \\
\hline J0809-5652 & K0.0 & $104 \pm 3$ & 14-12-01 & $\mathrm{H}$ & $0.782 \pm 0.005$ & $29.9 \pm 0.34$ & $2.73 \pm 0.01$ & $\mathrm{C}$ & 0.0051 & $81.2 \pm 2.4$ & 0.90 & 0.27 & 0.3 & no & . \\
\hline J0903-6348 & M0.5 & $66 \pm 3$ & 14-04-21 & $\mathrm{H}$ & $1.132 \pm 0.007$ & $64.1 \pm 0.34$ & $5.02 \pm 0.03$ & B & 0.0750 & $74.7 \pm 3.4$ & 0.67 & 0.04 & 0.1 & yes & $2,4,10$ \\
\hline J0931-5314 & K5.0 & $120 \pm 19$ & 15-05-12 & $\mathrm{H}$ & $0.264 \pm 0.002$ & $8.2 \pm 0.34$ & $3.48 \pm 0.10$ & $\mathrm{CO}$ & 0.0143 & $31.6 \pm 5.0$ & 0.76 & 0.14 & 0.2 & no & $\ldots$ \\
\hline \multicolumn{16}{|c|}{ ABDor } \\
\hline J0524-4223 & M0.5 & $52 \pm 9$ & 14-12-01 & $\mathrm{H}$ & $0.248 \pm 0.002$ & $61.0 \pm 0.37$ & $0.88 \pm 0.01$ & $\mathrm{CO}$ & 0.0000 & $12.9 \pm 2.2$ & 0.37 & 0.22 & 0.6 & yes & 2,4 \\
\hline \multicolumn{16}{|c|}{ TWA } \\
\hline J1234-4538 & M1.5 & $78 \pm 3$ & $15-05-12$ & $\mathrm{H}$ & $0.591 \pm 0.004$ & $309.2 \pm 0.34$ & $0.03 \pm 0.03$ & $\mathrm{CO}$ & 0.0000 & $46.2 \pm 1.8$ & 0.49 & 0.48 & 1.0 & yes & 11,12 \\
\hline
\end{tabular}

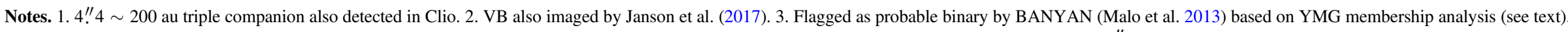

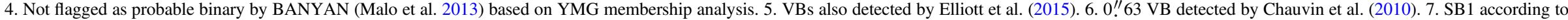

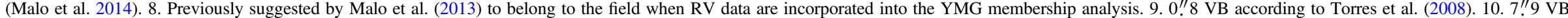
detected by Malo et al. (2014). 11. 0"7 VB suspected by Zuckerman et al. (2001). 12. 0." 62 VB resolved by Janson et al. (2012).

${ }^{a}$ Position angle (PA) is measured in degrees east of north

${ }^{\mathrm{b}}$ Typical uncertainties are $0.02 M_{\odot}$ (see Section 5.2).

(This table is available in machine-readable form.) 


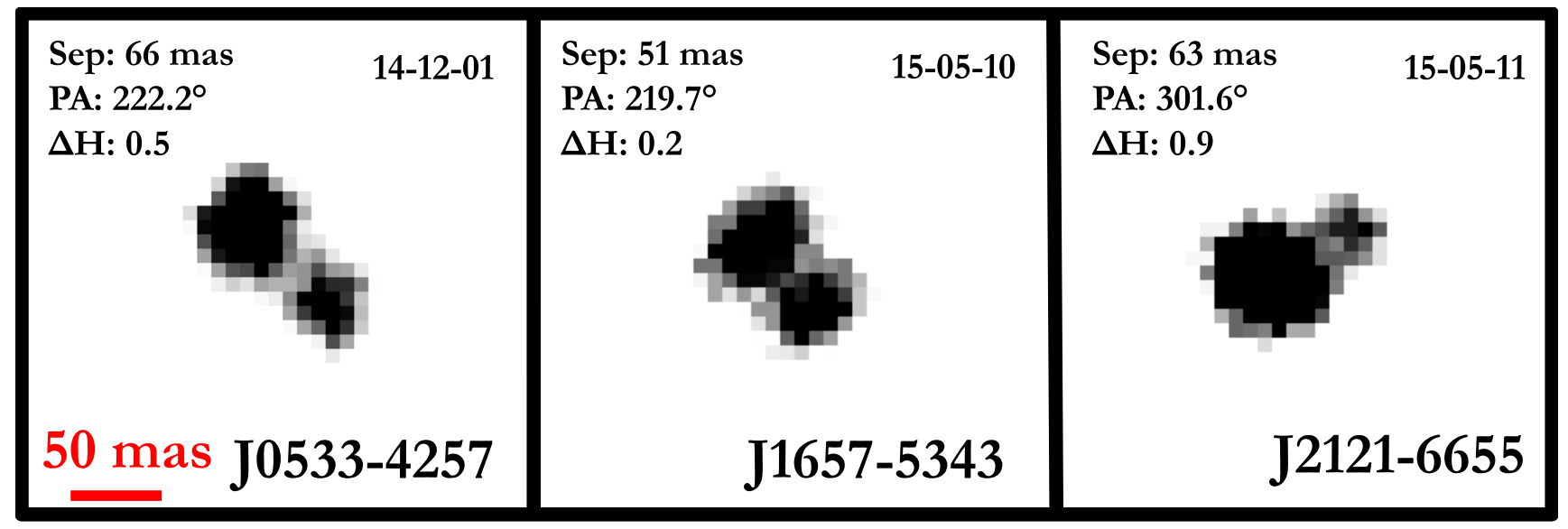

Figure 6. VisAO images of binaries with angular separations below 70 mas. Although the components are not cleanly separated in their Clio images, fits to these Clio images with two-source models yield robust measurements of their binary parameters. Each image is $280 \times 280$ mas. The images have been oriented so that north points up and east points left. The color scale is arbitrary for display purposes.

spectroscopic binary and treat it as such in the analysis. We do not detect a visual companion in this system, but most SBs are below our detection limits.

J0241-5259AB. We observe both targets to be tight binaries. This updates the multiplicity designation of J12 for the "B" component, which they observed to be single. The nominal A and B components are $\sim 21^{\prime \prime}$ apart. At their common distance of $43 \mathrm{pc}$, they may be part of the same system separated by $1000 \mathrm{au}$. Moreover, since each component is a tight binary in itself (this work), the system is potentially a hierarchical quadruple.

J0511-4903. J17 tentatively suggest the existence of a very faint $\Delta z^{\prime}=6.1$ companion at 2 !" 4 whose mass would be $0.015 M_{\odot}$. According to the Baraffe isochrones corresponding to the age of Columba, for a low-mass companion of this $\Delta z^{\prime}$, the expected $\Delta H$ is $\sim 4.5$. We do not observe such a companion in our short exposures, but we would not expect to. At this separation, these short Clio exposures are sensitive to $\Delta H \sim 4.3$ at $15 \sigma$ and $\Delta H \sim 4.8$ at $10 \sigma$.

J0903-6348. A comoving analysis shows that the $\sim 1$ !"1 "companion" detected in Clio is a background object (see Table 5). J17 also reports a detection of this companion, but their $z^{\prime}$-band measurement indicates that the spectral energy distribution (SED) rises toward the optical bandpasses, consistent with the hypothesis that it is a background object. In addition, there exists a wide (7!"9) companion (Malo et al. 2014; Janson et al. 2017). We include this system as a singlestar system in our statistical analysis, regardless.

$J 1234-4538$. This visual binary was first imaged by $\mathrm{J} 12$ in the $i^{\prime}$ and $z^{\prime}$ bands. Our additional epoch gives us a $\sim 5$-year baseline in total, providing astrometric confirmation (see Table 5 and Figure 15). Based on NIR fluxes ( $\Delta H=0.03$, Section 5.2), we determine the primary and secondary masses to be roughly equal at 0.49 and $0.48 M_{\odot}$, respectively. These are discrepant from the masses estimated by J12 $\left(0.355\right.$ and $\left.0.290 M_{\odot}\right)$, which are based partly on optical flux ratios $\Delta i^{\prime}=0.48$ and $\Delta z^{\prime}=0.51$. While the nature of this discrepancy is unclear, it may be because (1) J12 derived their masses from spectral types using relations calibrated for older field stars, and that the spectral type they use (M2.5) is different than that measured by Shkolnik et al. (2011) (M1.8), and (2) there exist systematic differences between models at this age, mass, and wavelength range. We opt to use our own inferred masses for consistency with the rest of our sample.
J1657-5343. We resolve this target into a $\sim 51$ mas binary (see Figure 6). J17 claims a possible detection of two substellar companions at $3 ! \prime 2$ and 3!"5 with $\Delta z^{\prime}=5.6$ and 6.59, respectively, but flags them as probable background objects. Their inferred masses are 0.03 and $0.02 M_{\odot}$. There is no evidence of these objects in Clio images, whose sensitivity at these separations is $\Delta H=7.5$, supporting the hypothesis that these are blue background contaminants.

J2149-6413. This object appears single in both the Clio and VisAO images taken on the night of 2015-05-11, for which the VisAO corrections are relatively poor. The minor axis of the halfmaximum surface has width 64 mas and the axis ratio is 0.89 , meaning that the single-source PSF is rather round. Attempted two-source fits did not find robust solutions. Chauvin et al. (2010) find that this is a nearly equal-mass $(\Delta K=0.2)$ binary with separation 74 mas in 2005. As in Sections 4.1 and 5.2, we use the $\Delta K$ measured from Chauvin et al. (2010) to calculate the primary mass to be 0.18 and the secondary mass $0.16 M_{\odot}$. At a distance of $44 \mathrm{pc}$, the projected separation corresponds to $3.3 \mathrm{au}$ and the period of this target, assuming equal-mass components $\left(M_{\mathrm{tot}}=0.18+0.16=0.34 M_{\odot}\right)$, would be $\sim 10$ years. We include this target in our calculations of multiplicity rates based on all known binaries, but not in the visual binary rate and the separation range and mass ratio distributions, which are based on our Clio detections only (see Section 5).

J2247-6920. We detect a faint visual companion at 0!'25 12 au with Clio. J17 observed the same target, but designated it as a single star. This object is also an SB1, and the RV amplitude of $>27 \mathrm{~km} \mathrm{~s}^{-1}$ suggests a tight binary (Malo et al. 2014), making the observed companion a tertiary. This object was originally noted by Malo et al. (2013) to be a possible field interloper. However, it is unclear whether the SB1 is corrupting the RV measurement. With a new parallax from Gaia, J17 reanalyzes this system using BANYAN and assigns it to the field. However, the Gaia distance appears to be consistent with the statistical distance prediction from Malo et al. (2013) assuming it is a TucHor member. We opt to keep J2247 in our sample.

\subsection{Comparison to BANYAN}

The Bayesian analysis for nearby young associatioNs (BANYAN, Malo et al. 2013; Gagné et al. 2014) is an algorithm that assigns to stars a statistical probability of 


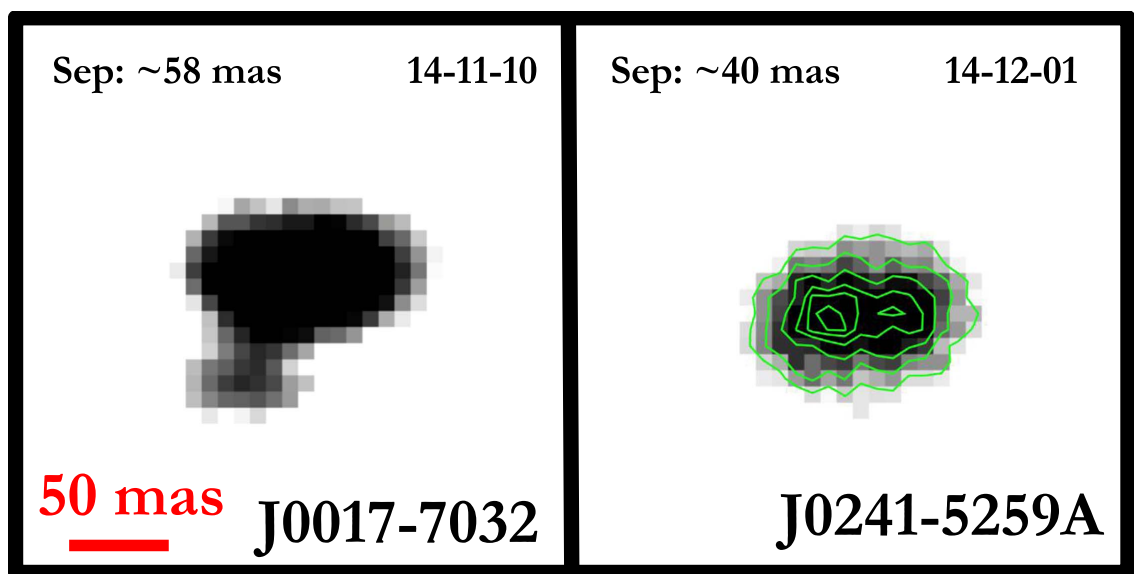

Figure 7. Visual binaries resolved through VisAO, band $z^{\prime}$. The images have been derotated such that north points up and east points left. The color scale is arbitrary for display purposes.

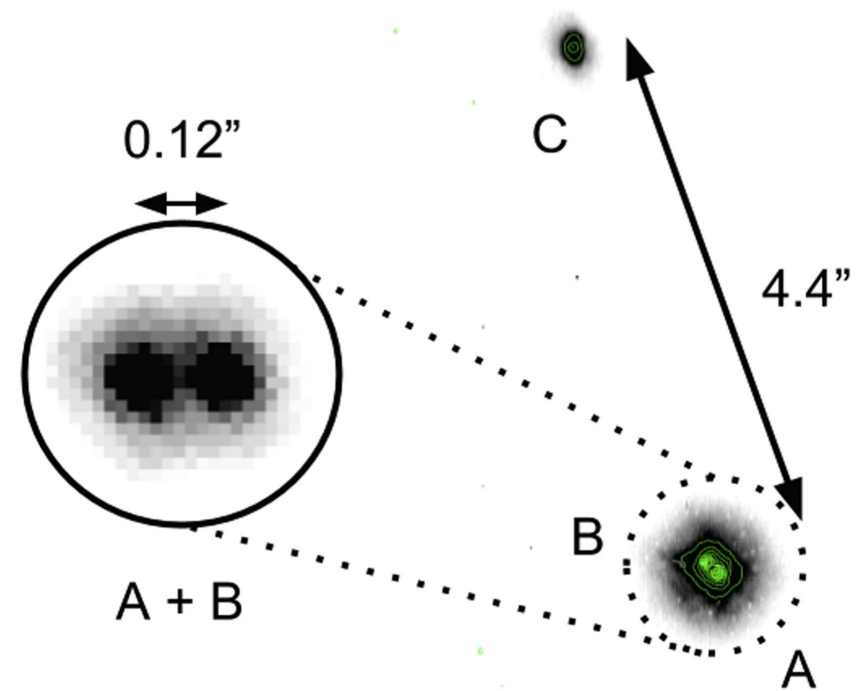

Figure 8. Triple system J0030-6236, with its tertiary companion at 4!" 4. The primary is itself a tight 0 !" 12 visual binary.

belonging to a given nearby YMG. It considers the hypothesis that a given object may be an unresolved equal-luminosity binary. If the binary solution gives a higher membership probability, then the object is flagged to reflect its possible binary nature. Malo et al. (2014) suggests that follow-up observations could be used to verify the BANYAN method for identifying binaries.

Sixty-four of the targets in our sample were drawn from Malo et al. $(2013,2014)$ and therefore have a BANYAN assessment of binarity. We observe 17 visual binaries with Clio and 2 additional binaries with VisAO. Of these, 5 and 2 , respectively, were flagged as possible binaries by BANYAN. BANYAN flags a further 10 systems as possible binaries. We do not observe any of these to be VBs. However, 1 system (J2149-6413, see Section 4.3) was identified by Chauvin et al. (2010) to be a binary and 3 more are confirmed SBs (J12314558: SB2; J1425-4113: SB2; J1524-4929: SB1; refer to Table 7). Of the remaining BANYAN objects in our sample that are not flagged as binaries, 4 are detected to be SB2s (J0533-5117 and J0451-4647 (Kraus et al. 2014), and J08187239 and J0846-7246 (Malo et al. 2014)). J2247-6920 is not only an unequal-mass visual binary (this work), but also an

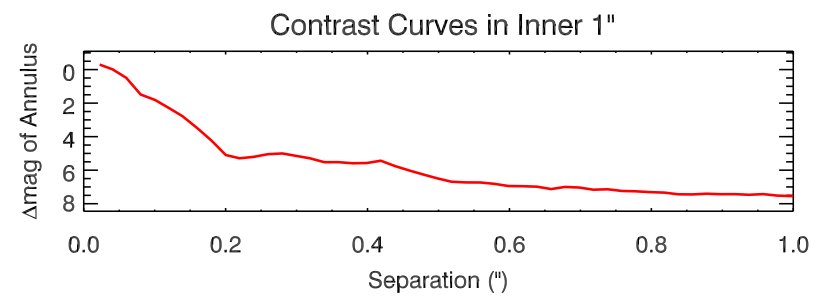

Azimuthal Slices of Contrast Curve Proxies at $45^{\circ}$ Slices

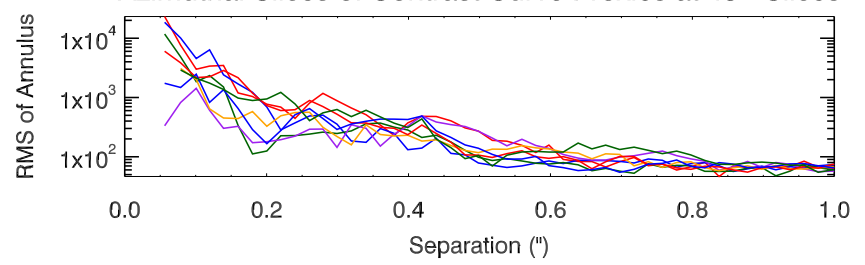

Figure 9. $15 \sigma$ contrast curves in the inner 1 "' for J0254-5108, a single star, is typical of that for our sample. Top panel: $15 \sigma$ contrast curve. Bottom panel: annulus rms curve in $45^{\circ}$ slices.

SB1 (Malo et al. 2014, see Section 4.3). In addition, we infer J0222-6022 to be an SB1 (see Section 4.3).

While we expect the BANYAN algorithm to miss pairs with a very high mass-ratio, it is unclear why it failed to identify nearly equal-mass binaries (e.g., J1516-5855, J0717-6311). It would be worthwhile to reassess the YMG membership probabilities for the new binaries presented in this work.

\section{Multiplicity Statistics}

We compute multiplicity statistics within our sample. For each statistic, we perform the analysis on two binary subsamples below 4"'0: 1) all known binaries (including SBs), and 2) visual binaries robustly fit by a two-source model in the Clio images.

\subsection{Contrast Curves and Observational Completeness Maps}

In order to study population statistics, we must first compute the completeness corrections by computing contrast curves for our targets. 
We calculate azimuthally averaged contrast curves using the formalism in Brandeker et al. (2006; see Equation (1) therein):

$$
\Sigma(R)=-2.5 \log \left[\frac{n \sigma_{\mathrm{pix}}(R) \sqrt{N_{\mathrm{PSF}}}}{F_{\mathrm{PSF}}}\right] \text { mag. }
$$

Here $R$ denotes distance from the primary star's centroid and $\Sigma$ $(R)$ denotes the limiting contrast for the secondary to be detected in terms of magnitudes. $n$ is the threshold $\mathrm{S} / \mathrm{N}$ corresponding to the detection method. $\sigma_{\text {pix }}(R)$ is the rms in an annulus of $R-\delta R$ to $R+\delta R$ around the primary. $N_{\mathrm{PSF}}$ represents the number of pixels within the FWHM of the primary PSF, and $F_{\mathrm{PSF}}$ is the integrated flux enclosed in the pixels. In the narrow mode, the Clio detector is $8^{\prime \prime} \times 16^{\prime \prime}$, so we are generally sensitive to companions occurring below $8 / 2=4^{\prime \prime}$ in all directions. For simplicity, we calculate the contrast curves to $4^{\prime \prime}$ and use that as our cutoff for companion detections.

For each processed image we use the best-fit triple bivariate Gaussian PSF solution for the primary to numerically compute $F_{\text {PSF }}$ and $N_{\text {PSF. }}$. If there is a companion, we subtract the PSF model of the companion before computing the contrast curve.

The chosen contrast threshold should result in reliable identification of true stellar companions. The most prevalent false sources are in the form of ghosts and speckles. To determine the appropriate threshold, for every target we constructed residual images by subtracting the empirical azimuthally averaged flux profile around the primary centroid. We searched for candidate sources that fell above the local annular rms in the residual image at $n-\sigma$ as follows: first, we isolated each pixel that met this criterion. We treated each pixel as if it could be the centroid of a source scaled from the fitted PSF of the primary, hence taking the pixel flux to be the peak flux of such a "source." We demand that within a square with a side length equal to the PSF half-maximum diameter around this pixel, $70 \%$ of all pixels are at least $50 \%$ as bright as the target pixel. For pixels that met the conditions thus far, we cross-matched them to within 3 pixels of the same position with respect to the primary in the other images for a given target and epoch. The last step is very effective in screening out bright ghosts associated with the instrument whose location depends on the dithering position. Inspection reveals that speckles sometimes exceed $\sim 10 \sigma$, but are virtually nonexistent at $\sim 15 \sigma$. Therfore, we chose $n=15$ for the contrast curves. Figure 9 shows a representative contrast curve constructed for a typical image. The lower panel shows that the dependence on azimuthal angle is weak.

Mawet et al. (2014) points out that speckles increase the noise level above the naive Gaussian assumption with $2-3 \lambda / D$ of the primary centroid. In contrast, Equation (1) assumes Gaussian noise, and so our computation of the contrast curves does not explicitly take this effect into account. At the same time, we do not expect this to have a substantial effect on our results because we choose our detection threshold based on the level of the speckle noise.

For each target, we averaged all the contrast curves for each epoch. We chose the best of the epochs for our detectability proxy. Here "best" is defined as the curve with the most sensitivity between 0 ." 1 and 1 ." 0 from the primary.

From these contrast curves we construct an observational completeness map for our sample. At any given $\Delta$ mag and log (separation), we use the contrast curves to calculate the fraction of stars in the sample for which a companion with these properties would be detectable. The completeness map in these observed parameters is displayed in the left panel of Figure 3.

\subsection{Calculation of Physical Parameters}

Physical projected separations are calculated using the reported distances to each target (Table 3 ). The primary mass is derived from the $2 \mathrm{MASS}$ photometry in conjunction with the latest stellar isochrones for PMS stars (Baraffe et al. 2015). If the star is a detected VB, then we divide the 2MASS magnitude between the components according to the measured flux ratio (see also Section 4.1). For the two binaries detected in VisAO and not Clio, there is not enough throughput for a reliable extrapolation from the observed visual flux ratio to the NIR flux ratio, thus we used the following approximation: when we judge the flux ratio to be high, we assigned all of the 2MASS flux to the primary; otherwise, we split the light equally between the two components, equivalent to obtaining the mass of each component from the system magnitude revised upward by 0.75 (see Table 6). When the target is a known SB (see Section 4.2), we take that information into account when calculating the mass. For SB1s, we assume that the primary is the dominant component in flux and mass and neglect any contribution from the secondary. For SB2s, unless an explicit measurement of flux and/or mass ratio is available, we assume an equal-mass binary. ${ }^{13}$ For J2149-6413 and the triple system J0030-6236, see Section 4.3.

For most targets, the dominant source of mass error comes from uncertainties in the distance, resulting in an error of $\sim 0.02 M_{\odot}$ on average. For comparison, typical uncertainties on 2MASS photometry and measured $\Delta$ mag $(\sim 0.03)$ correspond to an error of $\sim 0.005 M_{\odot}$. The youngest targets ( $<30 \mathrm{Myr}$ ) may be subjected to greater mass error due to uncertainty in age, since luminosity evolves most steeply at lower ages. For TWA (10 $\pm 3 \mathrm{Myr})$ and $\beta$ Pictoris $(24 \pm 3 \mathrm{Myr})$, the typical mass error from age indeterminacy can be up to $\sim 0.08 M_{\odot}$ and $\sim 0.03 M_{\odot}$, respectively. Inherent stellar model uncertainties can introduce greater errors and are briefly discussed in Section 5.6.6. We also note that Baraffe models are computed for solar abundances. Since young stars tend to be more metal rich on average, any metallicity dependence of the isochrones could introduce a systematic error in the inferred mass.

We then transform the observational completeness map into one in mass ratio and projected physical separation. To calculate the binary separations, we again use the reported distances to each target (Table 3 ). We convert each target's best contrast curve into one in the space of mass ratio versus projected physical separation. In the case of known unresolved binaries (J2149-6413, VisAO binaries, and SBs), we use the combined mass of the two components as the primary mass. Then, the mass ratios of hypothetical companions corresponding to set contrasts are presented relative to this combined mass (see Tables 6 and 7). Our outer detection limit of 4" set by the detector's field of view translates into a variable limit in projected physical separation. The resulting map is shown in the right panel of Figure 3. Our completeness to mass ratios greater than 0.2 between 10 and 100 au is $\sim 90 \%$. In general,

\footnotetext{
$\overline{13}$ These are admittedly crude assumptions, which may cause a systematic over- or underestimation of the primary masses of SB1s/SB2s, respectively. We evaluated the possible magnitude of these errors by recalculating the primary masses for all SB2s following the method for SB1s and vice versa and recomputing the statistics. The effect on the results is negligible.
} 

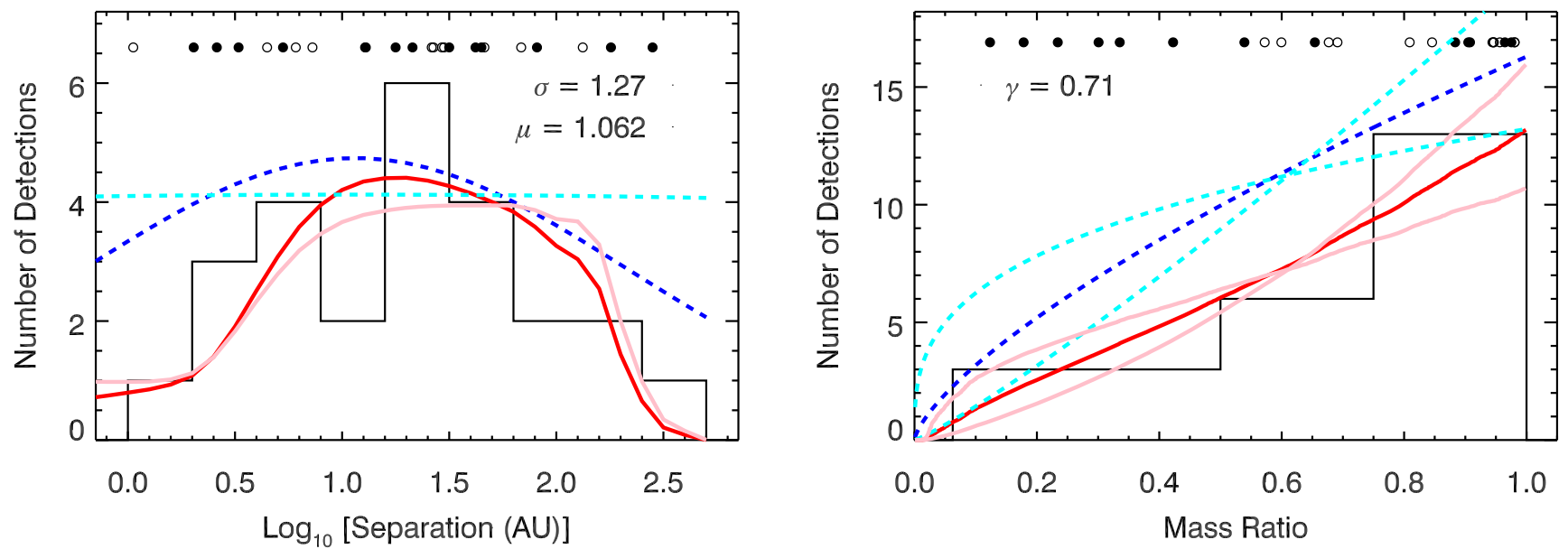

Figure 10. Left panel: distribution of projected physical separations for the Clio VBs. Black points at the top of the plot denote positions of individual detections, closed and open points denote those for which $M_{\text {prim }}>0.6$ and $<0.6 M_{\odot}$, respectively. The blue broken line is the underlying log-normal distribution fit to the detections, whereas the red solid line represents that of the incompleteness discounted (i.e., observed) distribution. Shown in cyan is a log-normal distribution with width $\sigma=10$, i.e., virtually flat. The pink curve is the predicted observation. The two scenarios are virtually indistinguishable. Right panel: distribution of mass ratios for the Clio VBs. A power law of form $q^{\gamma}$ is fit to the detections with the best-fit functions shown as blue dashed lines and its completeness-corrected version plotted in solid red. The cyan dashed lines represent the solutions whose likelihood deviates from the best fit by $1 \sigma$. The pink lines are corrected for detectability effects.

less massive primaries are fainter and allow for greater sensitivity to closer-in and lower mass companions.

Based on a lack of $q<1$ binary discoveries beyond 20 au in their sample of young M dwarfs, Daemgen et al. (2007) suggest that for M-dwarf primaries, there may be a desert of low-mass ratio companion occurrence beyond 25 au. In this work we have found 10 companions to M-type stars beyond 20 au with mass ratios between $\sim 0.5$ and 0.99 , hence it is unclear whether such a desert really exists.

\subsection{Integrated Multiplicity Fractions}

Out of the total sample of 104 imaged systems (after discarding J0102-6235, see Section 4.3), 25 are found to have an NIR visual companion whose projected separation lies between $\sim 0$ " 05 and 4".0 (Table 8). The widest companion in this angular separation range lies at $\sim 300 \mathrm{au}$. At least 1 of these systems also harbors SB signatures (J2247-6920). VisAO resolved 2 more binaries (Table 6). From the literature, a further 9 systems have known RV or close visual companions (Tables 7, J2149-6413). At least 2 of these systems may be triples (J0030-6236 and J2247-6920), and 1 might be a $\sim 1000$ au hierarchical quadruple. Counting the quadruple as 2 separate systems, the resulting measurement of the raw multiplicity fraction $\left(\mathrm{MF} \equiv N_{\text {multiple }} / N_{\text {all }}\right.$ ) for our sample is $36 / 104=35_{-4}^{+5} \%$ for all separations below $300 \mathrm{au}$, and $25 / 104=24_{-4}^{+5} \%$ for Clio VBs only.

When referring to $\mathrm{M}$ dwarfs for pre-main sequence stars, we are faced with a dilemma: should we designate objects as " $\mathrm{M}$ dwarfs" on account of spectral type or stellar mass? On the main sequence, the two are tightly correlated, hence can be used interchangeably (e.g., a M0 star has mass $\sim 0.6 M_{\odot}$; Baraffe \& Chabrier 1996). However, the correspondence is sensitive to age for pre-main sequence stars. While its mass stays relatively constant, a young star's observable spectroscopic parameters change with time during the PMS phase as it contracts toward the main sequence. Stellar mass is a more fundamental and physically meaningful parameter that is also relatively invariant with age. However, characterizing a system by spectral type is attractive because it is a direct observable and less sensitive to any binarity information to which we are ignorant. Hereafter, for any measurements made for the "Mdwarf subsample," we specify whether they refer to systems of the M-spectral type or with $M_{\text {prim }} \leqslant 0.6 M_{\odot}$, whichever is more appropriate.

The subsample of $\mathrm{M}$ dwarfs (by spectral type) exhibits a raw multiplicity rate of $26 / 87=30 \%$ below 300 au and $18 / 87=21 \%_{-4}^{+5}$ for visual companions identified in Clio imaging. For the sample of systems for which $M_{\text {prim }} \leqslant 0.6 M_{\odot}$, the rates are $23 / 64=36 \% \pm 6 \%$ and $17 / 64=27 \%{ }_{-5}^{+6}$, respectively.

To account for our detection incompleteness to binaries of particular mass ratios and physical separations, we compute the correction factor for each Clio VB detection as the inversedetectability evaluated at its location in the physical completeness map. After applying these corrections, the Clio VB rate for the total sample is $34 \% \pm 5 \%$. For the M-type subsample the VB multiplicity with this correction becomes $27 \% \pm 5 \%$. For the $M_{\text {prim }} \leqslant 0.6 M_{\odot}$ sample, the revised VB rate after accounting for detectability is $36 \% \pm 6 \%$. When all binaries are counted, the fractions are $42 \% \pm 5 \%$ for the entire Clio sample, $34 \% \pm 5 \%$ for $M$ stars, and $44 \% \pm 6 \%$ for $M_{\star} \leqslant 0.6 M_{\odot}$. Note that in this calculation we have explicitly excluded those VBs in regions with detectability $>0 \%$ that we resolved by VisAO (J0017-7032 and J0241-5259A) or noted in previous works (J2149-6413), but were missed by Clio, as they should have been accounted for in the detectability corrections.

\subsection{Distribution in Projected Physical Separation and Mass Ratio}

The upper left panel of Figure 10 shows the distribution of all the Clio visual binary discoveries between $0 . " 05$ and 4 ." 0 in $\log$-projected physical separation. We attempted to fit a lognormal function with mean $\mu$ and intrinsic width $\sigma$ to the detections, modified by the empirical completeness map from the right panel of Figure 3. Using a maximum likelihood prescription, we find the distribution to be both consistent with being log-normal and flat. Thus, we cannot make a meaningful 


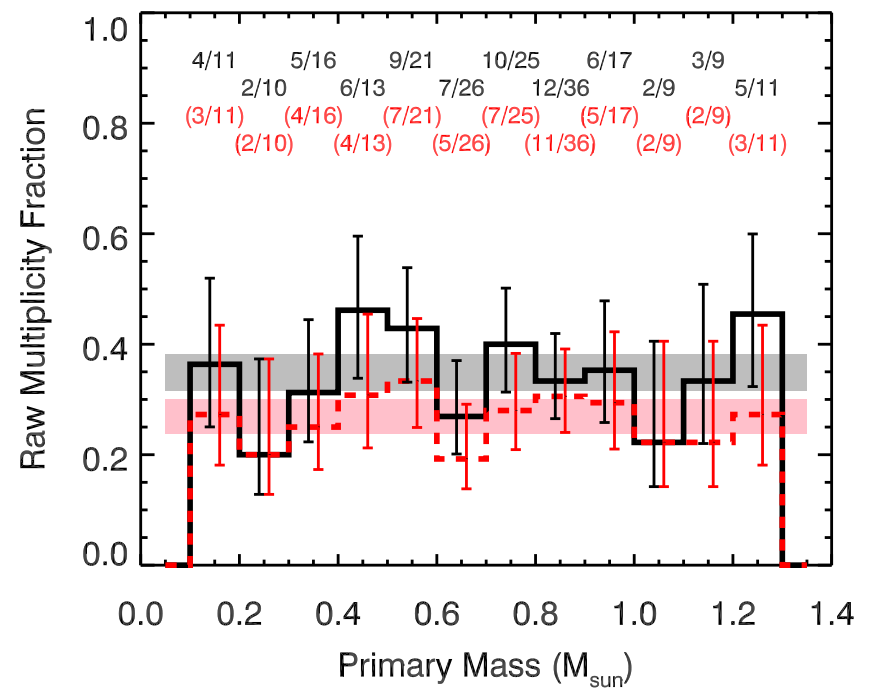

Figure 11. Raw multiplicity fraction of our joint YMG sample with SACY. The black solid histogram denotes multiplicity calculated from all known binaries (including VisAO and SBs) over the entire sample, whereas the red dashed histogram signifies the visual binary detections with physical projected separations between 1 and 300 au. $1 \sigma$ binomial confidence intervals are shown. Fractions near the top of the plot correspond to the number of total known multiple systems out of the number of systems observed, and in parentheses we provide the number of VBs (excluding J2149-6413 and VisAO binaries unresolved by Clio). The gray and pink shaded regions depict the average overall and visual multiplicities and their uncertainties across all masses.

comparison to the field, where FGKM binaries exhibit separations adequately described by a log-normal (e.g., Raghavan et al. 2010; Janson et al. 2012; Duchêne \& Kraus 2013; Ward-Duong et al. 2015).

The right panel of Figure 10 shows the mass ratio $(q)$ distributions of the Clio visual binaries. We fit a power law $\left(q^{\gamma}\right)$, modified by the physical completeness map in Figure 3, to each individual detection in a maximum likelihood framework. The best-fit $\gamma$ is $0.7 \pm 0.4$. A one-sample KolmogorovSmirnov test finds the $q$-distribution to be inconsistent with a uniform at a significance level of 0.01. It is apparent that there is a strong preference for equal-mass pairings in this sample. To compare, in the field, the mass distribution of FGK binaries mass can generally be characterized by $\gamma \sim 0.3-0.4$ (Duchêne $\&$ Kraus 2013). Of course, the mass for a stellar companion has a hard lower bound at the hydrogen-burning limit $\left(0.08 M_{\odot}\right)$. Therefore, when considering stellar companions, the lower bound on the relevant mass ratios increases for decreasing primary mass.

\subsection{Combined Analysis with SACY}

The SACY consortium has been actively studying the properties of stars in nearby YMGs (Torres et al. 2008). Elliott et al. $(2014,2015)$ present results from their multiplicity survey of SBs and visual binaries. SACY covers many of the same YMGs as in our work over a comparable separation range, but their objects are generally more massive-predominantly greater than $0.6 M_{\odot}$. Here we combine our samples (see Elliott et al. 2015, Tables 1 and 5) and repeat our calculation of multiplicity fractions. This joint analysis commands greater statistical power and covers an extended range in parameter space.

Of the 109 objects presented in the SACY survey, there are secure detections (i.e., bound probability exceeds 95\%) for 32 visual multiples, 5 of which are triples ( 3 visual triples, 2 visual binaries $+\mathrm{SB}$ ), and an additional $5 \mathrm{SBs}$. In the joint sample analysis, we use measurements from our campaign for the 9 targets that were also observed by SACY (2 VBs, $1 \mathrm{SB})$. Our mass, astrometry, and multiplicity measurements are consistent with SACY when accounting for the modest distance discrepancies (up to $5 \mathrm{pc}$ ) except in the following cases:

1. $V^{*}$ DZ Cha (J1149-7851): SACY assigns this star to $\eta$ Cha at a distance of 102.7 pc (see also Luhman 2007; Murphy et al. 2013), deriving a mass of $0.91 M_{\odot}$, while Malo et al. (2014) lists it under $\beta$-Pic at $68 \mathrm{pc}$, which is used to derive a stellar mass of $0.72 M_{\odot}$ in this work. This lower stellar mass is more consistent with the spectral type (M0-1V), therefore we use this mass and the Malo et al. (2014) YMG designation. Nevertheless, we acknowledge that youth signatures found by Murphy et al. (2013) argue in favor of the $\eta$-Cha interpretation. A Gaia parallax will settle this ambiguity.

2. CD-53 544 (J0241-5259A): this target is observed to be a 40 mas binary using VisAO imaging (see Table 6), which is below the imaging resolution of SACY.

3. TYC 8098-414-1 (J0533-5117): Kraus et al. (2014) identify this object as an SB2.

4. GSC 08350-01924 (J1729-5014) and CD-44 1173 (J0331-4359): small differences between our astrometry measurements for these objects can be explained by expected orbital motion over the time span of $\sim 2-10$ years separating our images from the SACY work (see Table 5 and Appendix). Our primary and secondary mass calculations are consistent with each other.

In total, for the joint sample of 204 targets, there are at least 52 visual multiples with projected separations above 75 mas, 6 visual binaries below 75 mas, 13 SBs that have no noted VB counterparts, and 7 triples, all of which also manifested as visual multiples. This gives a combined raw multiplicity fraction of $35 \% \pm 3 \%$ and a triple fraction of $3 \% \pm 1 \%$ for MKG dwarfs in YMGs. The companion fraction $\left(\mathrm{CF} \equiv\left(N_{\text {binary }}+2 N_{\text {triple }}+\ldots\right) / N_{\text {all }}\right)$ is then $38 \% \pm 3 \%$. The visual binarity rate is $28 \% \pm 3 \%$.

Figure 11 shows the raw multiplicity fraction of all known multiple systems (black) and that of detected visual multiples (red) for the joint sample of 204 systems spanning a primary mass range of $0.1-1.2 M_{\odot}$. In mass bins for which each survey contributes comparable numbers of targets $\left(0.6-0.8 M_{\odot}\right)$, the binarity rates we measure agree. Below $0.6 M_{\odot}$ the statistics are dominated by our campaign, whereas above $0.8 M_{\odot}$ they are chiefly determined by SACY. With the added statistical power in each mass bin and an extended range of masses, we still see no indication of deviation from flatness in the raw multiplicity fraction observed. This finding appears to extend the same conclusion from Elliott et al. (2015) for FGK dwarfs in YMGs down to $0.2 M_{\odot}$ (but see Section 5.6).

Figure 12 shows the fraction of multiple systems among the targets in our sample grouped by YMG membership and plotted against their ages for the joint sample. Once again, there does not appear to be a dependence on age or environment in the range spanned by this set of YMGs.

\subsection{Sources of Bias}

As with all population studies, a number of observational biases could have influenced our results and interpretations. In 

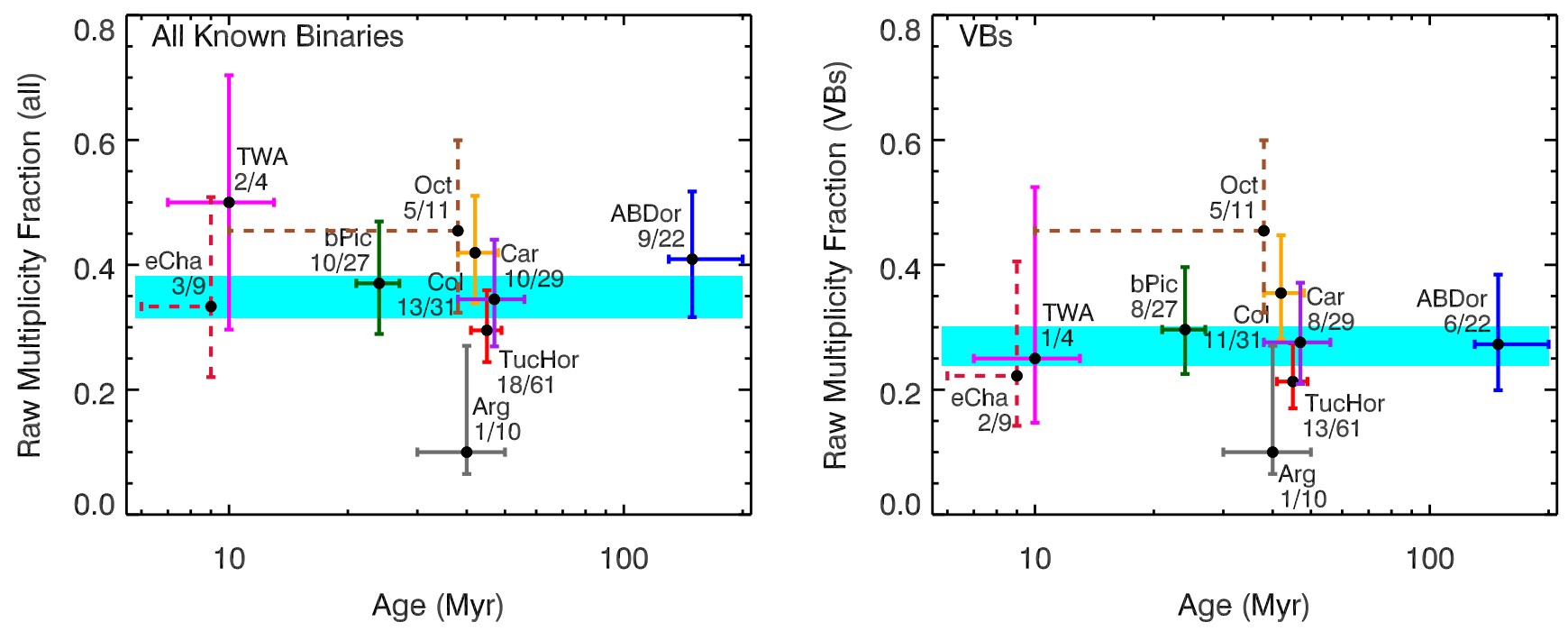

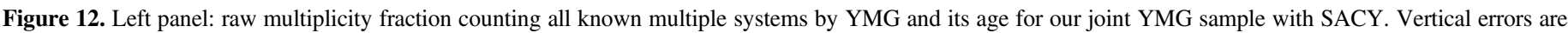

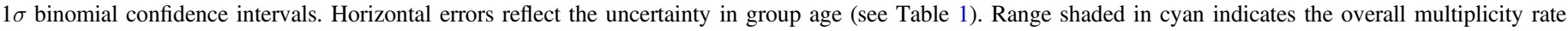

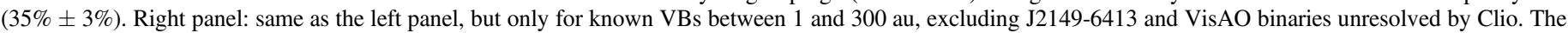

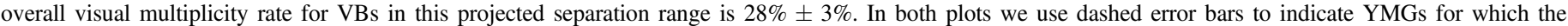
measurements come entirely from SACY.

Section 5.3 we have used completeness maps to correct the bias that is due to non-uniform sensitivity to the mass ratios and separation ranges of companions for each target. This is done for our assessments of the distributions in separation and mass ratio as well as for the overall visual binarity. In this subsection we discuss other relevant biases. Their net effects are summarized in Section 5.6.7.

\subsubsection{Branch Bias}

Branch (1976) cautioned that a key observational bias affecting binary statistics from magnitude-limited surveys is a systematic difference in the volume out to which binaries are visible as compared to single stars. The net outcome is a binary rate boosted by a factor dependent on the intrinsic binarity rate as well as the underlying distribution of companion flux ratios (see, e.g., Burgasser et al. 2003; Section 6.1, Equations (4)(5)). The relative enhancement is greater for populations with lower binarity and those consisting of systems biased toward equal-mass pairings. Similarly, it tends to inflate a population's mass ratio distribution at the high end. Furthermore, in general we expect the bias to affect $\mathrm{M}$ dwarfs most severely because they are intrinsically fainter.

However, it is difficult to evaluate a quantitative Branch boost factor for our study. This is in part due to our relative ignorance about the intrinsic mass ratio distribution of our target population, but also because our sample is not strictly magnitude limited - sample selection is primarily motivated by membership in YMGs. Compared to a magnitude-limited study in uniform space, the Branch bias should be markedly weaker for populations that have a finite spatial extent tangentially and radially, as for YMGs. This is because the increased volume for binaries from larger distances goes as $r$ rather than $r^{3}$. Furthermore, when there exists an upper bound in distance, stars that are brighter than the threshold survey magnitude even at the far end of the population will not suffer from this bias at all. This implies that earlier-type stars are systematically less affected than later-type stars. Below we attempt to estimate the possible effect of Branch bias in our multiplicity measurement.
There are two ways in which Branch bias could manifest in our study. First, we need to examine whether such bias may already be present in the target source catalogs. None of the catalogs claim to be complete, and it is difficult to assess their intrinsic selection biases. To address the level of their completeness to low-mass stars, we ask the following: whether the catalogs document a substantial population of members down to M4 in each YMG (they do), and whether the later types are dominated by known multiples over singles (they are not, except in distant Carina). We conclude that catalog bias is probably subdominant to the next source of Branch bias.

The other source of Branch bias is associated with our own target selection strategy, which has prioritized brighter objects and resulted in the majority of observed stars being $K<10$.

To understand this bias for our specific selection of YMGs and target spectral types, we constructed toy models of our YMGs at their corresponding ages and distances. We distributed young low-mass star systems, a controlled fraction of which are equal-mass binaries, in a Gaussian around the average YMG distance. The Gaussian widths are defined such that $2 \sigma$ incorporates the range of distances spanned by the known group members. After computing their apparent $K$ magnitudes using Baraffe models (Baraffe et al. 2015), we calculated the ratio of injected to observed binary fraction ( $F \equiv f_{\text {obs }} / f_{\text {true }}$ ) for a given limiting $K$-magnitude. Based on the magnitudes of the observed targets, we set the limiting magnitude to be $K<10 \mathrm{mag}$, and injected $f_{\text {true }}=0.3$.

We arrive at the following estimates: the mass-weighted mean $F$ for each YMG is lower than 1.3 over masses $0.2-0.6 M_{\odot}$, and the overall weighted mean is $\sim 1.16$. In all groups, there is no bias at the upper end of the mass bracket $\left(>0.5 M_{\odot}\right)$. Of course, the Branch effect is more pronounced for lower masses. Carina is most extreme, where in our toy model $F \sim 3$ for $M_{\star}=0.2 M_{\odot}$, followed by TucHor and Columba at $F \sim 2.3$. The rest have $F<1.6$ for $M_{\star}=0.2 M_{\odot}$. In reality, only $6 \%$ of our survey targets are drawn from Carina. Since all six targets are relatively massive $\left(>0.4 M_{\odot}\right)$, Carina does not actually contribute to the low-mass Branch bias. Nevertheless, in the lowest mass range 
$\left(0.1-0.3 M_{\odot}\right)$ the mean $F$ is still relatively high at $\sim 1.8$, which is due to the high representation of TucHor members in our sample.

It is worth remembering that the estimates above are conservative in the sense that the toy models only include equal-mass binaries. Branch bias is reduced for a realistic population with a range of mass ratios. Furthermore, Branch bias is not only a function of a star's intrinsic brightness (therefore mass and age), but is also highly dependent on the geometry of a population's spatial distribution.

In sum, we expect Branch bias to inflate the average overall M-dwarf multiplicity measured here by no more than a multiplicative factor of 1.25 (i.e., requires multiplying the observed rate by 0.8 to correct). However, a downward adjustment factor of $<0.6$ may be needed in the lowest mass bins.

\subsubsection{Selection Bias}

One obvious bias affecting observed multiplicity is the sample selection bias. As described in Section 2, the initial sample was culled for Malo et al. (2013) binaries for which components have been separately resolved and have individual spectral types identified. The net effect of such a selection bias is to depress the observed binarity.

We can estimate the extent to which our measurement is affected by this selection bias, as follows. We assemble the full pool of M-type candidate YMG members with declinations below $-40^{\circ}$ from the five source catalogs (see Section 2) without regard to binarity. All but one (J1814-3246) of our M-type targets have $\delta<-40^{\circ}$. There are 192 objects in this pool, 86 of which have visual binarity constraints provided by Clio imaging (excluding J0102-6235, see Section 4.3). Nineteen other candidates were part of J12's AO campaign, screened for visual binarity as close as $0 . " 075$, which found 13 more multiples. Another 34 objects were observed by J17, who identified a further 9 visual multiples in addition to $2 \mathrm{VBs}$ and 2 SBs already noted by Malo et al. (2014). In addition, we have identified two more binaries (J03210395-6816475 and $\mathrm{J} 20143542-5430588$ ) in the full sample pool based on guider camera imaging.

For the 51 remaining objects for which there are no binarity constraints available, including J0102-6235, we have assigned to them an "average" $<300$ au multiplicity equalling the overall rate derived from our own M-star subsample $(34 \pm 5 \%)$. After accounting for Branch bias $(34 \% \times 0.8=27 \%$, see Section 5.6.1), we get an additional $51 \times 0.27=13.8$ binaries. In total, the multiplicity rate for the YMG M-type sample pool becomes $[13+9+2+2+13.8+26] / 192=34 \%$. This is $\sim 4 \%$ higher than the raw value of $30 \%$.

We conclude that sample selection bias has an appreciable effect on our raw multiplicity fraction, and independent of other biases, it means that our measured value of the raw multiplicity may be underestimated by another additive factor of $\sim 4 \%$.

\subsubsection{Separation Distribution Bias}

Both SACY and our own multiplicity survey are sensitive to a limited range of projected separations, meaning that we are potentially probing only a subset of all binaries. The fraction we are missing depends on the underlying separation distribution.

In the field, the separation distribution is a function of primary mass. The average G-dwarf binary tends to be farther separated than an M-dwarf binary. When fitted with a unimodal lognormal function, the peaks are $\sim 45$ au and $\sim 5$ au, respectively. The distribution for $\mathrm{G}$ dwarfs is also broader, with a significant number of binaries lying between $10^{3}$ and $10^{6}$ au (e.g., Duchêne $\&$ Kraus 2013). Therefore, probing a fixed range of separations for all masses may introduce a systematic skew in the space of multiplicity versus primary mass.

Our search for companions to KM dwarfs is effectively confined to under $\sim 300$ au. The binaries in our sample have a comparable separation distribution to the field, with most of the density confined within $300 \mathrm{au}$ (see Figure 10). Therefore, if the true distribution is field-like, we expect our low-mass multiplicity measurement to be representative of the total multiplicity.

The SACY VB study (Elliott et al. 2015) detects companions to FGK-dwarf members between 3 and $1000 \mathrm{au}$. This is supplemented by the SACY SB study (Elliott et al. 2014) for enhanced completeness at shorter periods. Using kernel density estimation, SACY measures a separation distribution for their sample that is significantly narrower than the field, extending negligibly beyond $1000 \mathrm{au}$. If YMG G-dwarf binaries really have this narrower range of separations, then SACY would be essentially complete as well.

Nevertheless, Elliott et al. (2015) cautions that bias against wider companion discovery may exist in the algorithm they use to identify bound systems in the survey, which could render their derived separation distribution too narrow. If we instead assume a field-like separation distribution for Sun-like stars in YMGs, then SACY has missed $\sim 20 \%$ of all multiple systems from the wide end. Therefore, a factor of up to 1.25 may need to be applied to correct for the total multiplicity fraction for the higher mass bins.

\subsubsection{Membership Bias}

The methods by which memberships are determined for these targets are statistical and include any RV information available for the target, attributed to its galactic motion. Therefore, SB1s have a higher chance of being assigned to the wrong YMG or the field.

In assembling our sample, we have largely consulted the Malo et al. (2013) and Kraus et al. (2014) catalogs, which comprise $60 \%$ and $30 \%$ of the targets observed, respectively. Malo et al. (2013) give a list of all candidates whose YMG membership is assigned exclusively based on photometry and proper motion, since in general the targets had no RV information available at the time. Malo et al. (2014) update the properties and memberships of a subset of Malo et al. (2013) candidates using RV. While these properties are used in our analysis whenever possible, our decision to observe and include a particular Malo et al. (2013) target in our sample is not based on this new information. Kraus et al. (2014) collect single-epoch RVs for 205 possible members of TucHor. They reject $20(\sim 10 \%)$ from TucHor membership on the basis of incompatible RV, but caution that some may be bona fide SB1 members. Such targets are excluded from our sample. If half of the excluded TucHor members turn out to be SB1s (i.e., 5\%), we would expect a $1 \%-2 \%$ increase in the raw multiplicity rate calculated for our sample. It is possible that misassignment of stars to the incorrect YMG could affect our evaluation of the environmental/age dependence of multiplicity. As long as the SB1 bias is not systematically undermining a specific YMG, there should be no net effect. Since Kraus et al. (2014) 


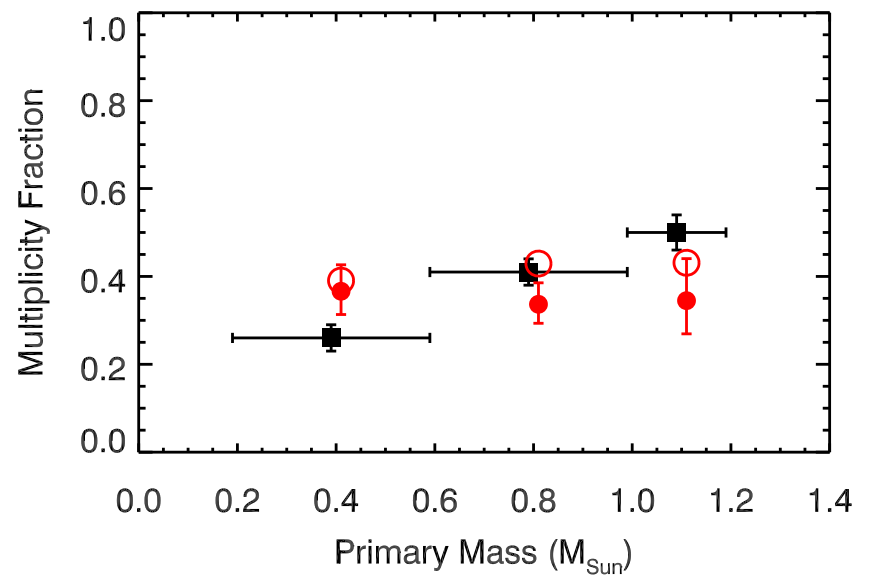

Figure 13. Comparison of multiplicity fractions as a function of primary mass between the field and that measured for YMGs in this study. Black squares denote field points (Duchêne \& Kraus 2013). Red solid circles represent raw measurements from our combined sample with SACY, and red empty circles are the post-bias correction values to our measurements. The bias-corrected values in the higher mass bins agree well with the field. However, the low-mass bin differs mildly.

contributes $\sim 70 \%$ of the TucHor sample, TucHor is the most affected by this bias, and its true multiplicity might be underestimated (by 3\%-4\% under the previous assumption).

Since the TucHor catalog of Kraus et al. (2014) only includes objects for which a spectrum has been acquired, one possible concern is that this sample could be biased toward bright targets. In reality, the objects in the Kraus et al. (2014) sample have $K$-magnitudes spanning from 6 to 11, a brightness range that is very representative of the overall sample. Therefore, we conclude that the effect of any RV selection bias has been subsumed under the Branch bias discussion in Section 5.6.1.

\subsubsection{Chance Alignment Bias}

A visual binary could appear to be single if its components are chance aligned along our line of sight. Unlike semimajor axis, projected separation is not an intrinsic property of a binary system, but a function of orientation and time of observation. Depending on the orbital elements of the system, binary components whose semimajor axes are theoretically resolvable may become momentarily chance aligned, i.e., their projected separation may drop below the resolution limit, in an arbitrary snapshot. On the surface, this phenomenon constitutes a potential source of bias that results in systematically missing binaries.

Upon closer inspection, the issue is more subtle. Such a bias is only problematic if we were interested in retrieving the true distribution of semimajor axes in our sample, which is difficult in practice. The semimajor axis is only statistically related to the projected separation measured at any give moment (see, e.g., Duquennoy \& Mayor 1991; Section 5.3; Torres 1999; Figure 3; Brandeker et al. 2006; Appendix). While the average projected separation is within a factor on the order of unity to the true semimajor axis, a large dispersion exists.

Therefore, although the semimajor axis is a more meaningful quantity to characterize a binary system, we do not attempt to perform a statistical retrieval. Nevertheless, we do investigate the potential chance alignment bias for a fixed range of semimajor axes to which observations are most sensitive, namely 3-300 au. Owing to the range of possible orbital eccentricities (e.g., a thermal distribution where $f(e)=2 e$ ), overall we expect comparable quantities of visual binaries to be scattered in as out by this bias, for a negligible net effect.

\subsubsection{Stellar Model Uncertainties}

Current state-of-the-art stellar evolution models tend to overpredict the radii (hence luminosity) of young low-mass stars by $10 \%-25 \%$ compared to empirical measurements (e.g., David et al. 2016). This is a source of systematic error in any derivation of stellar mass from luminosity. As the error acts differentially on stars of different masses, it could also affect mass ratio calculations of binary components, and therefore our physical contrast curves and estimates of incompleteness.

To examine the magnitude of such a bias, we consider the case of an M-dwarf binary for which one star is radiative $\left(0.5 M_{\odot}\right)$ and the other fully convective $\left(0.2 M_{\odot}\right)$. We expect any differential effect on mass ratio to be most pronounced for such a pairing, since the mass-luminosity scaling relation changes across the fully convective boundary. For a given overestimation factor in stellar luminosity of $30 \%$ for both components, the offset in mass ratio is only $\sim 5 \%$. Since $30 \%$ is already a conservative upper bound in the model luminosity error, we conclude that its impact on our physical sensitivity curves would be minuscule.

\subsubsection{Net Effect of Biases}

Figure 13 compares the multiplicity fraction in the field versus our YMG measurements as a function of primary mass. We divide the mass range spanned by the combined sample into three bins: $0.2-0.6,0.6-1.0$, and $>1.0 M_{\odot}$. The field values for the two higher mass bins are supplied by Raghavan et al. (2010), whereas the lowest bin is an average from the literature computed by Duchêne \& Kraus (2013). For YMGs, the lowest mass bin consists predominantly of MagAO stars, whereas the highest mass bin comprises objects almost exclusively from SACY. SACY targets also account for just over $60 \%$ of the intermediate-mass bin. In addition to the raw measured value, this plot shows a crude correction representing the combined effect of biases discussed.

In the low-mass bin, we begin by applying the detectability corrections (Section 5.3), which constitutes a 6\% additive boost. Then, we multiply the resulting fraction by the Branch bias factor of 0.8 (see Section 5.6.1). Finally, we add an upward correction of $4 \%$ for selection bias. The overall increase is $\sim 2 \%$.

In the intermediate-mass bin, we compute detectability corrections to the Clio targets and assume perfect sensitivity in this mass range from the SACY survey. The Branch bias is negligible in this mass regime. We add only $2 \%$ for selection bias because Clio targets contribute only $\sim 40 \%$ to this mass bin. Last, we multiply the multiplicity fraction by factor 1.1 assuming a $\sim 10 \%$ contribution from binaries wider than our detection range.

In the highest mass bin, which is composed of SACY targets exclusively, we apply a correction factor of 1.25 to account for the contribution from wide binaries, as discussed in Section 5.6.3).

After correction, multiplicity rates in the higher mass bins agree well with the field rates. However, the YMG M-dwarf binary rate has been increased by the bias corrections to greater $(>1 \sigma)$ than the field rate. Nevertheless, since the corrections are 

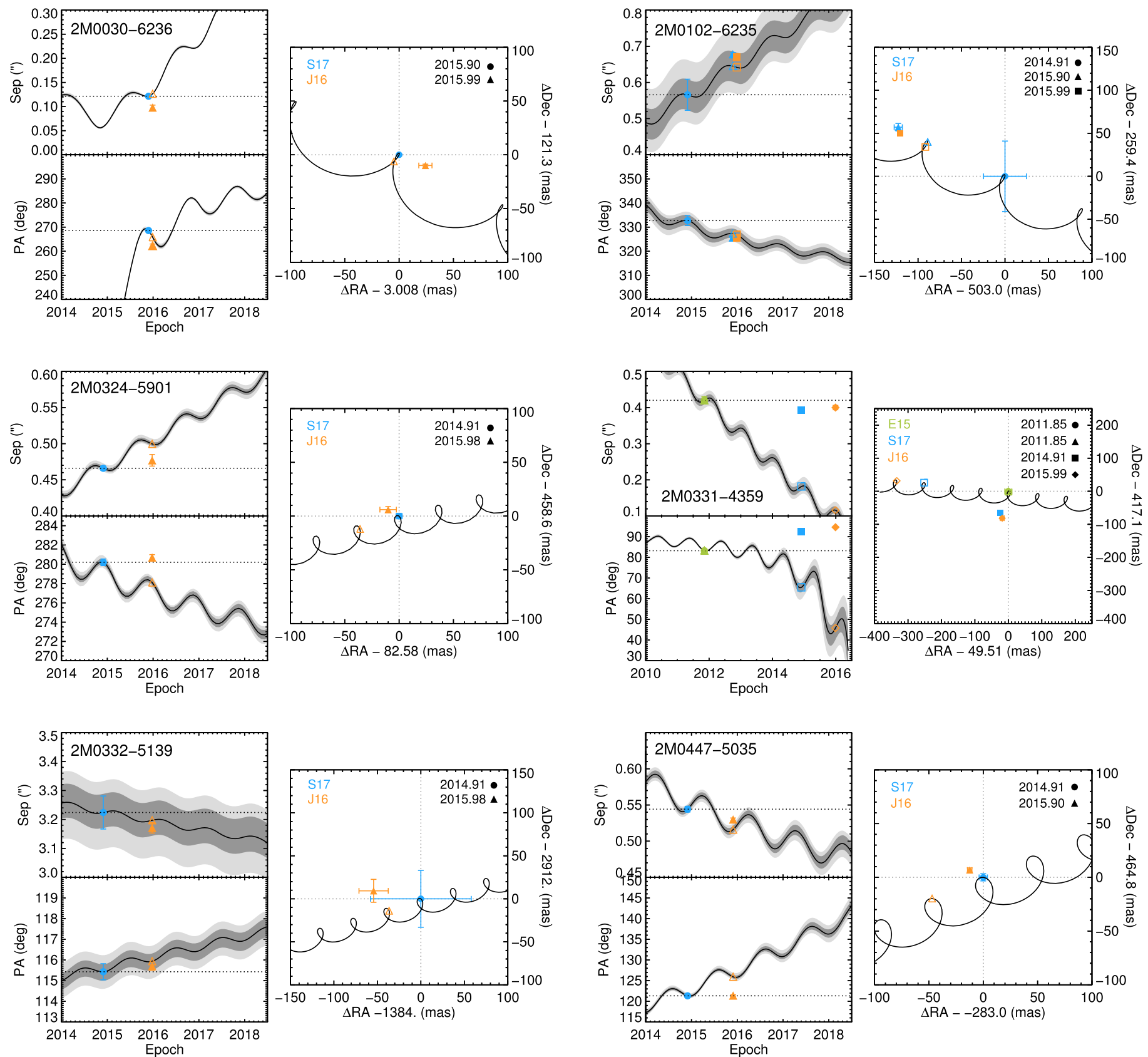

Figure 14. Comoving analysis for Clio visual binaries with multi-epoch data. Each set of three plots displays the analysis for the target whose name appears in the upper left corner. C10: Chauvin et al. (2010), J12: Janson et al. (2012), E15: Elliott et al. (2015), J17: Janson et al. (2017), and S17: this work. References and epochs of the data points plotted are listed in the right panel plot. Left: separation (top left) and position angle (bottom left) of the companion. The solid line shows the expected astrometric track of a distant background object as a result of proper and parallax motion of the primary, and open symbols indicate the expected measurement for this background object. The gray shaded regions represent $1 \sigma$ and $2 \sigma$ errors in the background tracks based on uncertainties in the proper motion, distance, and first-epoch astrometry. The dotted lines show a perfectly comoving track. A real companion should not follow the background track and should move minimally relative to the primary (i.e., follow the dotted lines). Any movement between the epochs should be adequately explained by orbital motion. Right: $\Delta$ R.A. and $\Delta$ decl. as seen on the sky ( $\Delta$ refers to primary-secondary position).

relatively crude and the discrepancy of limited significance, the YMG findings may still be reconcilable with the field measurements.

\section{Discussion and Conclusions}

In this paper we have summarized our work to identify unresolved binaries in recently proposed low-mass members of local YMGs using NIR AO imaging. The campaign resulted in images of 27 visual binaries, 15 of which were previously unknown, between 0 ". 05 and 4 ". 0 (roughly 3 to $300 \mathrm{au}$ ) in a sample of $104 \mathrm{KM}$-dwarf stars associated with 7 YMGs with ages from 10 to $200 \mathrm{Myr}$. For 25 systems we have measured astrometric and photometric properties, deriving stellar masses and projected physical separations. When combined with the literature, 18 visual doubles now have multi-epoch and multiwaveband data. Two additional suspected visual binaries (and J0102-6235 and J0903-6348) are rejected on astrometric grounds, and the rest are verified. In conjunction with spectroscopic binarity information from literature, we also find 

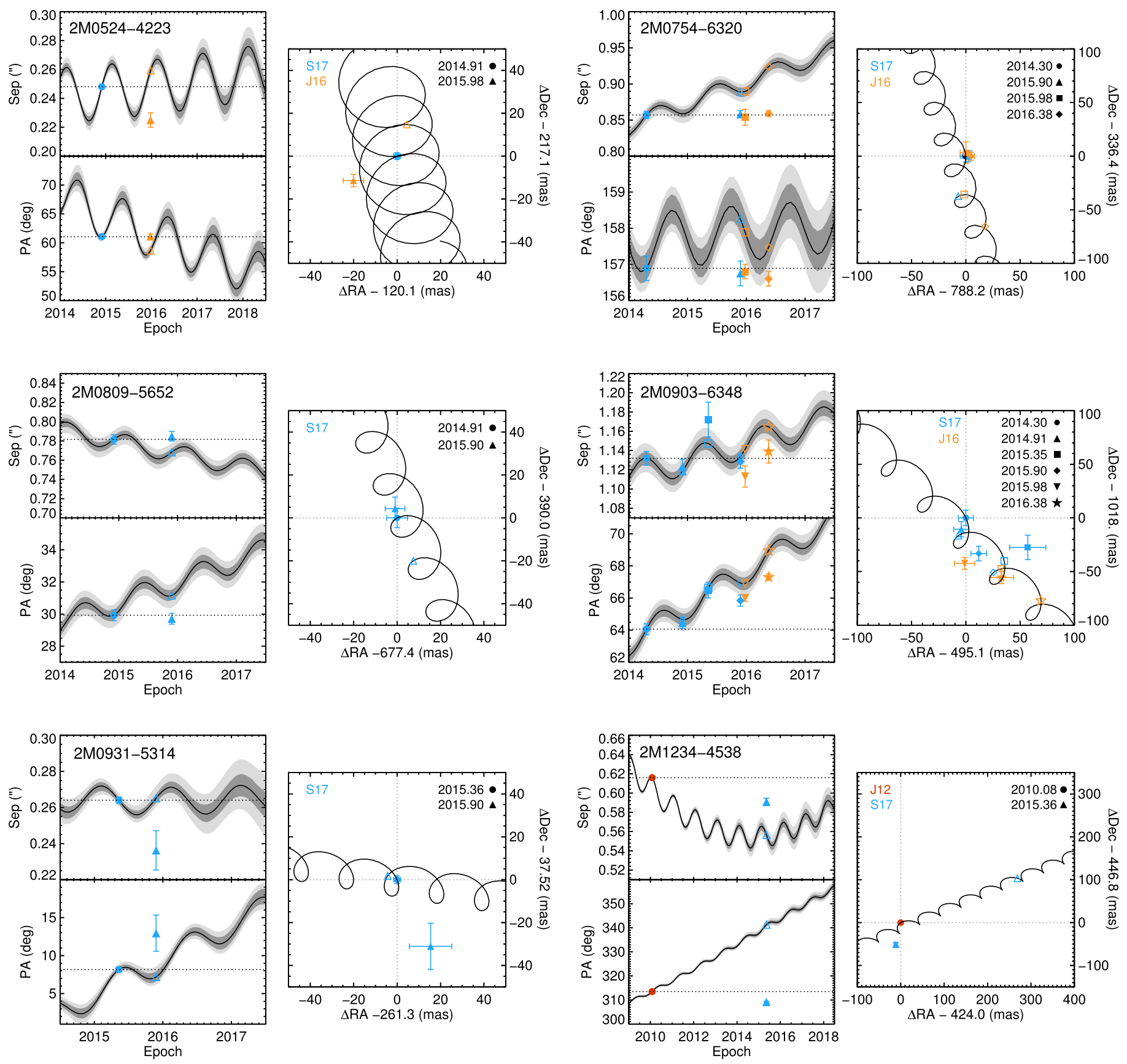

Figure 15. Same as in Figure 14.

that two of systems are in reality triples (J0030-6236 and J2247-6920), and two of the binaries potentially form a hierarchical quadruple (J0241-5259A and B).

We have measured standard multiplicity statistics for this population in concert with SACY's GK dwarfs (Torres et al. 2008; Elliott et al. 2014, 2015, 2016). In so doing, we generated a broader picture of stellar multiplicity for YMG stars in the mass range of 0.1 and $1.2 M_{\odot}$, roughly doubling the mass range and number of targets from previous efforts. The 58 visual multiples and 12 spectroscopic binaries known from the literature with no visual counterparts in the joint sample of 204 stars gives a combined raw multiplicity rate of at least $35 \% \pm 3 \%$ with separations below 1000 au. The raw visual binarity rate $(1-1000 \mathrm{au})$ is at least $28 \% \pm 3 \%$, and the companion fraction is $38 \% \pm 3 \%$. These are comparable to field FGK stars for the same separation range (Raghavan et al. 2010; Duchêne \& Kraus 2013). Seven of the targets are triples, giving a triple rate of 3\% for the joint YMG GKM population.

Our M-star subsample has an overall raw observed binarity rate of $30_{-4}^{+5} \%$ under $300 \mathrm{au}$, and the raw rate of occurrence for visual binaries resolved by the Clio camera between 1 and $300 \mathrm{au}$ is $21_{-4}^{+5} \%$. The rates for stars with $<0.6 M_{\odot}$ are $36 \% \pm 6 \%$ and $27_{-5}^{+6} \%$, respectively. While the visually resolved raw binarity appears to be roughly consistent with the most recent field measurement for $\mathrm{M}$ dwarfs over comparable separation ranges $(\mathrm{CF} \sim 23 \% \pm 3 \%$ for 3-10,000 au; WardDuong et al. 2015), the overall multiplicity fraction is discrepant (MF 26\% $\pm 3 \%$ overall; Duchêne \& Kraus 2013). 

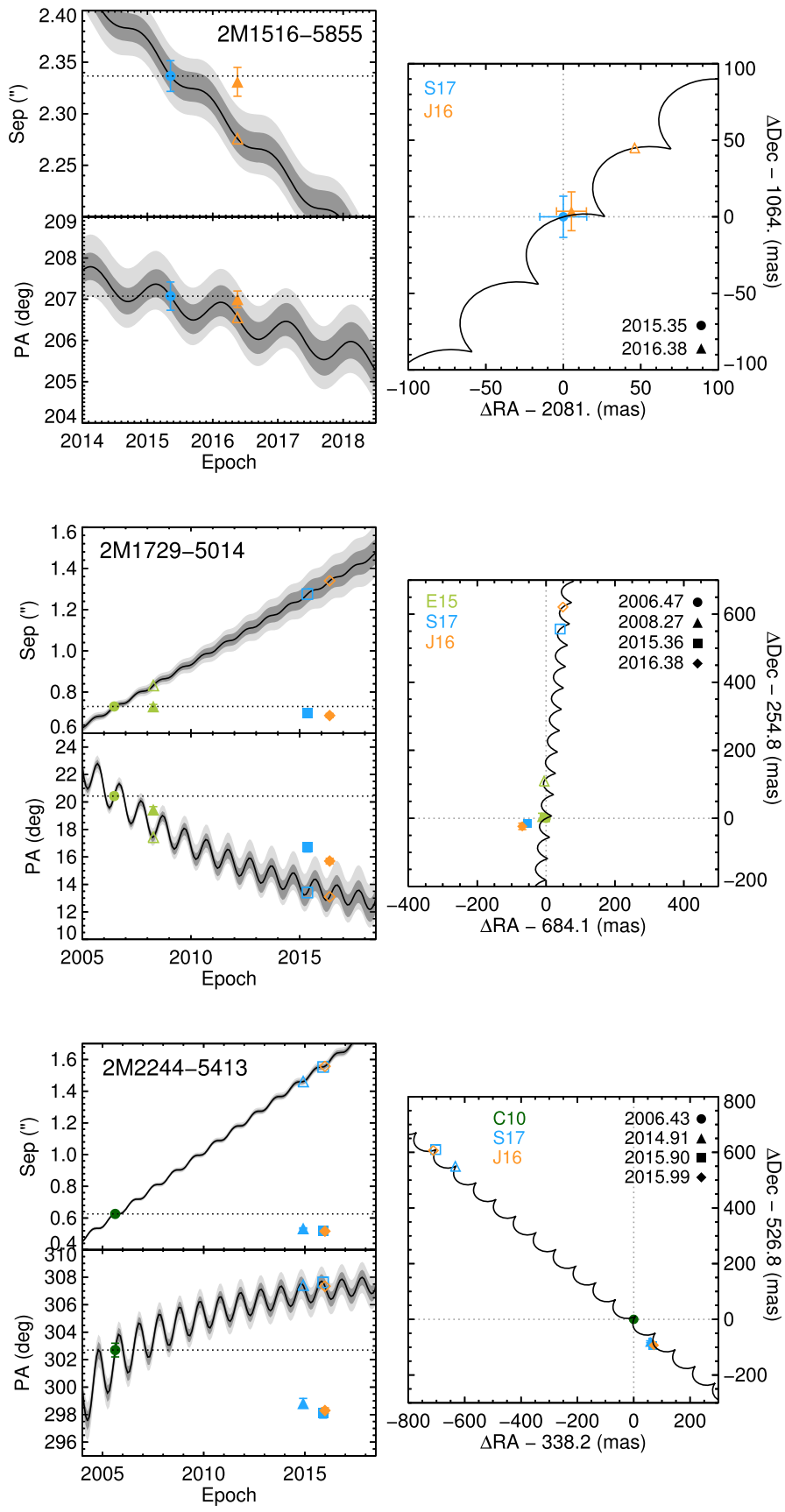
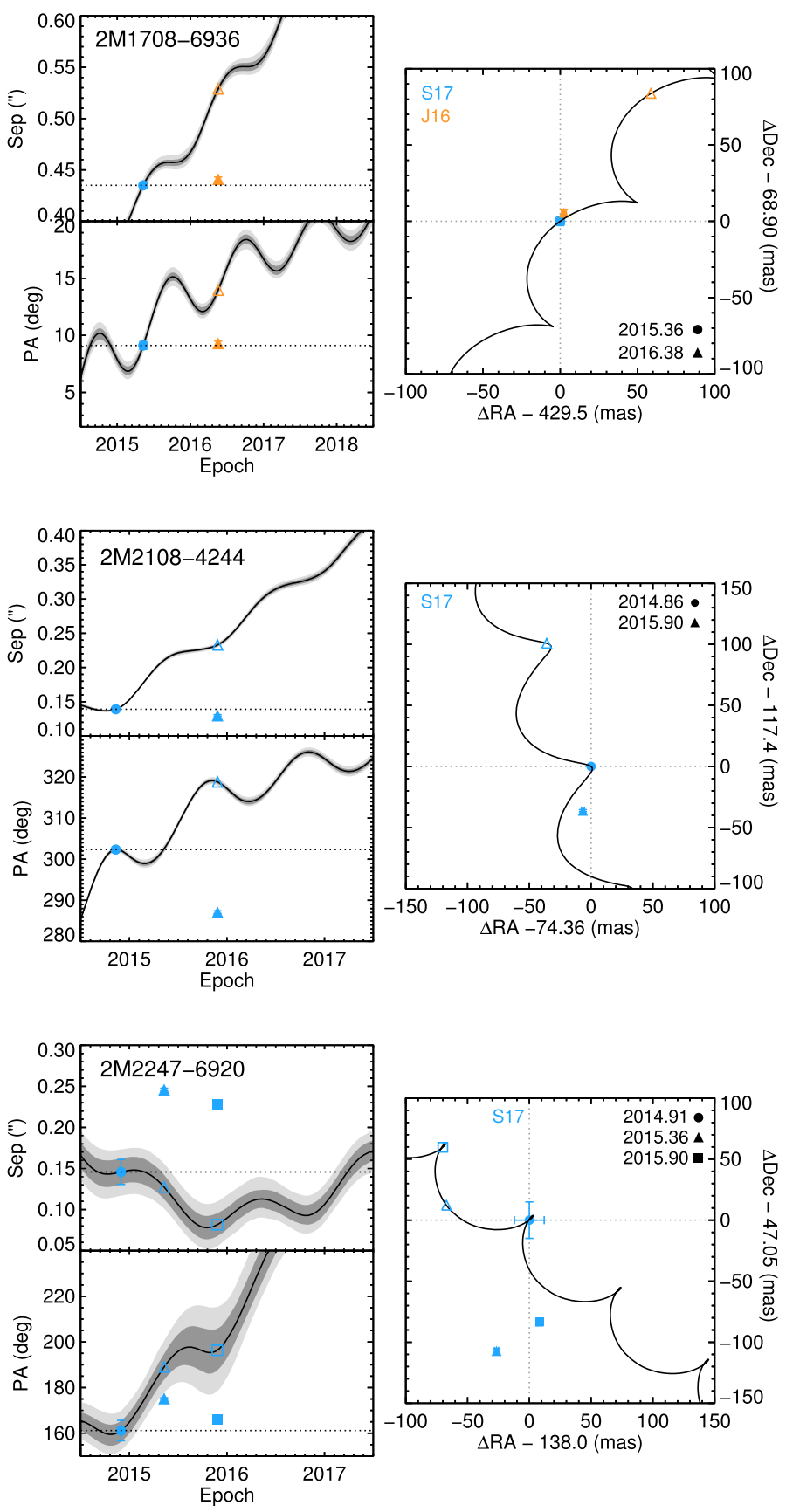

Figure 16. Same as in Figure 14.

Our combined analysis with SACY finds no evidence for the overall multiplicity fraction to be dependent on primary mass from 0.2 to $1.2 M_{\odot}$ in the raw measurements. This would corroborate the findings of SACY (Elliott et al. 2015), now with the statistics appreciably bolstered in the mass range of $0.2-0.7 M_{\odot}$. However, such a finding is contradicts the wellknown behavior in the field, where more massive primaries are more likely to be multiple.

As discussed in depth in Section 5.6, the raw observed multiplicity rates can deviate from the true rates as a result of various biases. Detectability considerations (Section 5.3) and initial selection bias against known visual binaries (Section 5.6.2) could have rendered our measurement for M-star multiplicity an underestimate from the true rate by $\sim 10 \%$. Meanwhile, Branch bias (Section 5.6.1) could inflate our observed M-dwarf binarity by up to a factor of 1.25 , and even more at the low-mass end. On the other hand, when separation bias (see Section 5.6.3) is accounted for in the measurement of higher-massed SACY objects, the values for GK dwarfs are boosted. The bias-adjusted multiplicities in the higher mass bins are consistent with those in the field, while in the low-mass bin there is still a difference at $\sim 1.5 \sigma$, which is subjected to various assumptions in corrections (see Figure 13 in Section 5.6.7).

For systems with M- to F-dwarf primaries, there is no significant indication of varying multiplicity fractions at $M_{\mathrm{tot}}>0.2 M_{\odot}$ between YMGs, despite their differences in age and possibly birth environments. Likewise, for this sample, no notable changes in binary separations are found as a 
function of age, although the statistics are based on small numbers and for $M_{\mathrm{tot}}>0.2 M_{\odot}$. These results agree with the YMG solar-type star sample of SACY and suggests minimal dynamical evolution in the $\sim 10-200 \mathrm{Myr}$ after their formation even for binary systems of lower mass, and supports a similar formation pathway for all YMGs.

Previous studies have used the binary fraction as evidence for the contribution of a given population to the field. For example, Patience et al. (2002) compared the field binary separation distribution with that of open clusters and T-associations, concluding that dense and loose star-forming environments contribute $70 \%$ and $30 \%$ of the field stars, respectively. Goodwin (2010) points out that when deducing the origin of the field from comparing binary statistics, one must consider the effect of dynamical processing on young populations, which could modify their binary rates before they enter the field. If the low-mass stars in YMGs do exhibit greater multiplicity than field stars and are expected to retain their multiplicity properties due to lack of dynamical evolution before entering the field, then YMGs may not be a significant contributor to the field. This agrees with the assessment by Lada \& Lada (2003) that most field stars originate in OB associations.

In the process of compiling this work, the authors have engaged in helpful discussions with numerous friendly and knowledgeable persons in various capacities. We would like to thank Sean Andrews, Gaspard Duchêne, Jason Eastman, Dan Foreman-Mackey, Robin Gong, Vinay Kashyap, Rainer Köhler, Charles Lada, Lison Malo, Ilya Mandel, Evgenya Shkolnik, and Willie Torres for their patience, insights, assistance, and encouragement.

We thank Adam Kraus for detailed feedback on an early version of this paper.

We also thank the anonymous referees for helpful suggestions.

Y.S. is supported by a Doctoral Postgraduate Scholarships from the Natural Science and Engineering Research Council (NSERC) of Canada. Support for B.P.B. was provided by NASA through Hubble Fellowship grant HST-HF2-51369.001A awarded by the Space Telescope Science Institute, which is operated by the Association of Universities for Research in Astronomy, Inc., for NASA, under contract NAS5-26555. Work by B.T.M., K.M.M., and J.R.M. were performed under contract with the Jet Propulsion Laboratory (JPL) funded by NASA through the Sagan Fellowship Program executed by the NASA Exoplanet Science Institute. L.A.C. was supported by CONICYT-FONDECYT grant number 1171246 and the Millennium Science Initiative (Chilean Ministry of Economy), through grant Nucleus RC130007. H.C. acknowledges support from the Spanish Ministerio de Economía y Competitividad under grant AYA 2014-55840-P. K.M.M.'s and L.M.C.'s work is supported by the NASA Exoplanets Research Program (XRP) by cooperative agreement NNX16AD44G.

This publication makes use of data products from the Two Micron All Sky Survey, which is a joint project of the University of Massachusetts and the Infrared Processing and Analysis Center/California Institute of Technology, funded by the National Aeronautics and Space Administration and the National Science Foundation. It also makes use of data products from the Wide-field Infrared Survey Explorer, which is a joint project of the University of California, Los Angeles, and the Jet Propulsion Laboratory/California Institute of Technology, funded by the National Aeronautics and Space Administration. The Digitized Sky Survey was produced at the Space Telescope Science Institute under U.S. Government grant NAG W-2166. The images of these surveys are based on photographic data obtained using the Oschin Schmidt Telescope on Palomar Mountain and the UK Schmidt Telescope. The plates were processed into the present compressed digital form with the permission of these institutions.

\section{Appendix \\ Astrometric Plots for Multi-epoch Data}

We present plots (Figures 14-16) depicting our astrometric analysis for Clio visual binaries with multi-epoch data to determine whether they are comoving or stationary background objects (Table 4).

\section{ORCID iDs}

Jennifer C. Yee (1) https://orcid.org/0000-0001-9481-7123

Brendan P. Bowler (1) https://orcid.org/0000-0003-2649-2288

Lucas A. Cieza (1) https://orcid.org/0000-0002-2828-1153

Benjamin T. Montet (1) https://orcid.org/0000-0001-

7516-8308

Héctor Cánovas (1) https://orcid.org/0000-0001-7668-8022 Michael C. Liu (ㄱ) https://orcid.org/0000-0003-2232-7664 Laird M. Close (단 https://orcid.org/0000-0002-2167-8246 Jared R. Males (i) https://orcid.org/0000-0002-2346-3441

Katie M. Morzinski (i) https://orcid.org/0000-0002-1384-0063 Katherine B. Follette (i) https://orcid.org/0000-0002-

7821-0695

\section{References}

Azulay, R., Guirado, J. C., Marcaide, J. M., et al. 2015, A\&A, 578, A16 Baraffe, I., \& Chabrier, G. 1996, ApJL, 461, L51

Baraffe, I., Homeier, D., Allard, F., \& Chabrier, G. 2015, A\&A, 577, A42 Bell, C. P. M., Mamajek, E. E., \& Naylor, T. 2015, MNRAS, 454, 593 Bowler, B. P., \& Hillenbrand, L. A. 2015, ApJL, 811, L30

Bowler, B. P., Liu, M. C., Mawet, D., et al. 2017, AJ, 153, 18

Branch, D. 1976, ApJ, 210, 392

Brandeker, A., Jayawardhana, R., Khavari, P., Haisch, K. E., Jr., \& Mardones, D. 2006, ApJ, 652, 1572

Burgasser, A. J., Kirkpatrick, J. D., Reid, I. N., et al. 2003, ApJ, 586, 512 Chauvin, G., Lagrange, A.-M., Bonavita, M., et al. 2010, A\&A, 509, A52 Cieza, L. A., Padgett, D. L., Allen, L. E., et al. 2009, ApJL, 696, L84 Daemgen, S., Siegler, N., Reid, I. N., \& Close, L. M. 2007, ApJ, 654, 558

David, T. H., Hillenbrand, L. A., Cody, A. M., Carpenter, J. M., \& Howard, A. W. 2016, ApJ, 816, 21

Delorme, P., Gagné, J., Gigrard, J. H., et al. 2013, A\&A, 553, L5

Donaldson, J. K., Weinberger, A. J., Gagné, J., et al. 2016, ApJ, 833, 95

Duchêne, G., \& Kraus, A. 2013, ARA\&A, 51, 269

Duchêne, G., Simon, T., Eisloffel, J., \& Bouvier, J. 2001, A\&A, 379, 147

Duquennoy, A., \& Mayor, M. 1991, A\&A, 248, 485

Elliott, P., Bayo, A., Melo, C. H. F., et al. 2014, A\&A, 568, A26

Elliott, P., Bayo, A., Melo, C. H. F., et al. 2016, A\&A, 590, A13

Elliott, P., Huélamo, N., Bouy, H., et al. 2015, A\&A, 580, A88

Gagné, J., Lafrenière, D., Doyon, R., Malo, L., \& Artigau, É. 2014, ApJ, 783,121

Goodwin, S. 2010, RSPTA, 368, 851

Gaia Collaboration 2016, A\&A, 595, A2

Janson, M., Durkan, S., Hippler, S., et al. 2017, A\&A, 599, A70 (J17)

Janson, M., Hormuth, F., Bergfors, C., et al. 2012, ApJ, 754, 44 (J12)

Jayawardhana, R., Coffey, J., Scholz, A., Brandeker, A., \& van Kerkwijk, M. H. 2006, ApJ, 648, 1206

Jeffries, R. D., Jackson, R. J., Franciosini, E., et al. 2017, MNRAS, 464, 1456 Joye, W. A., \& Mandel, E. 2003, in ASP Conf. Ser. 295, Astronomical Data Analysis Software and Systems XII, ed. H. E. Payne, R. I. Jedrzejewski, \& R. N. Hook (San Francisco, CA: ASP), 489 
Köhler, R., Kunkel, M., Leinert, C., \& Zinnecker, H. 2000, A\&A, 356, 541

Kraus, A. L., Ireland, M. J., Hillenbrand, L. A., \& Martinache, F. 2012, ApJ, 745,19

Kraus, A. L., Ireland, M. J., Huber, D., Mann, A. W., \& Dupuy, T. J. 2016, AJ, 152,8

Kraus, A. L., Shkolnik, E. L., Allers, K. N., \& Liu, M. C. 2014, AJ, 147, 146

Lada, C. J., \& Lada, E. A. 2003, ARA\&A, 41, 57

Lafrenière, D., Jayawardhana, R., van Kerkwijk, M. H., Brandeker, A., \& Janson, M. 2014, ApJ, 785, 47

Leinert, Ch., Zinnecker, H., Weitzel, N., et al. 1993, A\&A, 278, 129

López-Santiago, J., Montes, D., Crespo-Chacón, I., \& Fernández-Figueroa, M. K. 2006, ApJ, 643, 1160

Luhman, K. L. 2007, ApJS, 173, 104

Males, J. M., Close, L. M., Morzinski, K. M., et al. 2014, ApJ, 786, 32

Malo, L., Artigau, É., Doyon, R., et al. 2014, ApJ, 788, 81

Malo, L., Doyon, R., Lafrenière, D., et al. 2013, ApJ, 762, 88

Markwardt, C. B. 2009, in ASP Conf. Ser. 411, Astronomical Data Analysis Software and Systems XVIII, ed. D. A. Bohlender, D. Durand, \& P. Dowler (San Francisco, CA: ASP), 251

Mawet, D., Milli, J., Wahhaj, Z., et al. 2014, ApJ, 792, 97

Montet, B. T., Bowler, B. P., Shkolnik, E. L., et al. 2015, ApJL, 813, L11

Moór, A., Szabó, Gy. M., Kiss, L. L., et al. 2013, MNRAS, 435, 1376
Morzinski, K. M., Close, L. M., Males, J. R., et al. 2014, Proc. SPIE, 9148, 914804

Morzinski, K. M., Males, J. R., Skemer, A. J., et al. 2015, ApJ, 815, 108

Murphy, S. J., Lawson, W. A., \& Bessell, M. S. 2013, MNRAS, 435, 1325

Patience, J., Ghez, A. M., Reid, I. N., \& Matthew, K. 2002, AJ, 123, 1570

Raghavan, D., McAlister, H. A., Henry, T. J., et al. 2010, ApJS, 190, 1

Rodriguez, D. R., Zuckerman, B., Kastner, J. H., et al. 2013, ApJ, 774, 101

Shkolnik, E. L., Liu, M. C., Reid, N., Dupuy, T., \& Weinberger, A. J. 2011, ApJ, 727, 6

Skrutskie, M. F., Cutri, R. M., Stiening, R., et al. 2006, AJ, 131, 1163

Torres, C. A. O., Quast, G. R., Melo, C. H. F., \& Sterzik, M. F. 2008, in Handbook of Star Formation Regions, Vol. II, ed. B. Reipurth (San Francisco, CA: ASP)

Torres, G. 1999, PASP, 111, 169

Ward-Duong, K., Patience, J., De Rosa, R. J., et al. 2015, MNRAS, 449, 2618

Weinberger, A. J., Anglada-Escudé, G., \& Boss, A. P. 2013, ApJ, 762, 118

Wright, E. L., Eisenhardt, P. R. M., Mainzer, A. K., et al. 2010, AJ, 140, 1868 Yee, J. C., \& Jensen, E. L. N. 2010, ApJ, 711, 303

Zacharias, N., Finch, C. T., Girard, T. M., et al. 2012, yCat, 1322, 0

Zacharias, N., Monet, D. G., Levine, S. E., et al. 2005, yCat, 1297, 0

Zuckerman, B., \& Song, I. 2004, ARA\&A, 42, 685

Zuckerman, B., Webb, R. A., Schwartz, M., \& Becklin, E. E. 2001, ApJL, 549, L233 